\title{
Deaminative Arylation of Amino Acid-derived Pyridinium Salts
}

Megan E. Hoerrner, Kristen M. Baker,${ }^{\ddagger}$ Corey H. Basch, ${ }^{\ddagger}$ Earl M. Bampo, Mary P. Watson* Department of Chemistry and Biochemistry, University of Delaware, Newark, DE 19716 mpwatson@udel.edu

$\$$ These authors contributed equally.

\section{Supplementary Information}

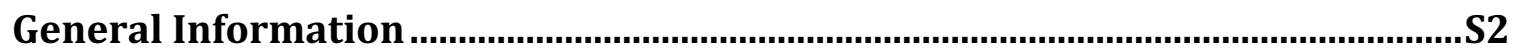

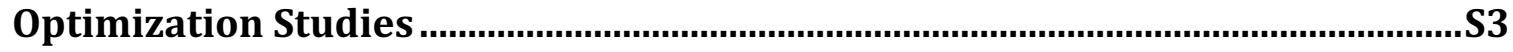

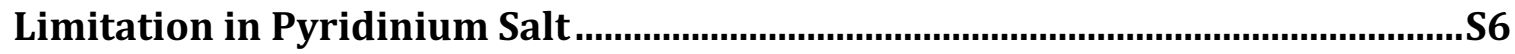

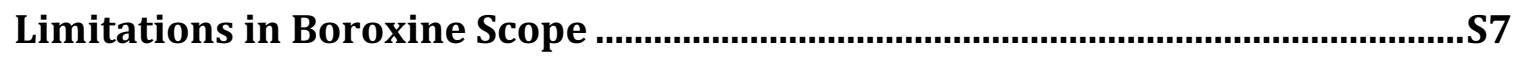

Suzuki-Miyaura Cross-Couplings of Amino Acid Pyridinium Salts ......................S7 General Procedure A: Cross-Coupling with Aryl Boroxines ....................................................S7

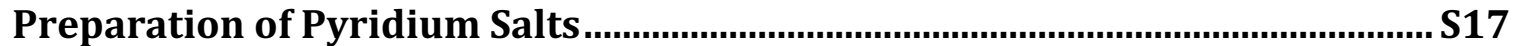

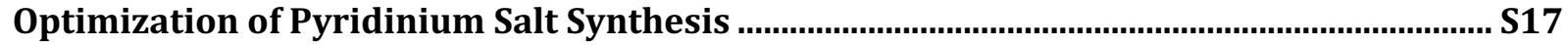

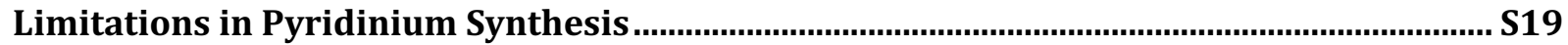

General Procedure B: Conversion of Amino Esters to Pyridinium Salts .............................. S20

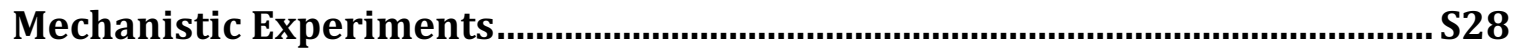

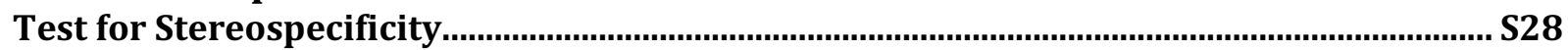

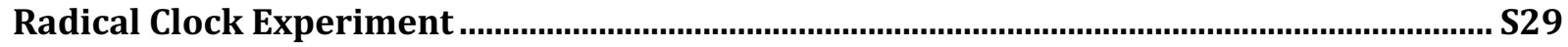

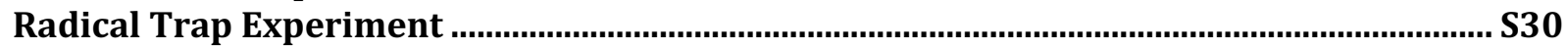

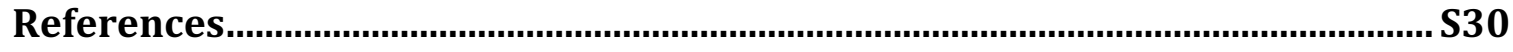

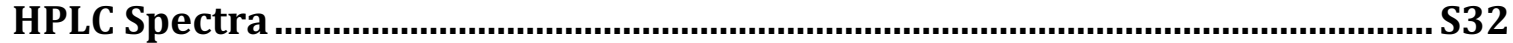

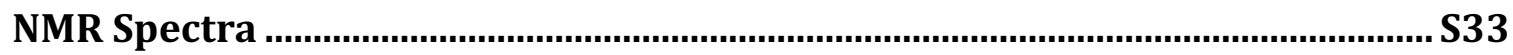




\section{General Information}

Reactions were performed in oven-dried round-bottomed flasks unless otherwise noted. Roundbottomed flasks were fitted with rubber septa, and reactions were conducted under a positive pressure of $\mathrm{N}_{2}$. Stainless steel syringes or cannulae were used to transfer air- and moisture-sensitive liquids. Flash chromatography was performed on silica gel $60(40-63 \mu \mathrm{m}, 60 \AA)$ unless otherwise noted. Commercial reagents, including 2,4,6-triphenylpyrylium tetrafluoroborate and the amino acids (or corresponding hydrochloride salts and methyl esters), were purchased from Sigma Aldrich, Acros, AstaTech, Fisher, Strem, TCI, Combi Blocks, Alfa Aesar, AK Scientific, Bide Pharmatech, Oakwood, or Cambridge Isotopes Laboratories and used as received with the following exceptions: $\mathrm{MeCN}$ and $\mathrm{CH}_{2} \mathrm{Cl}_{2}$ was dried by passing through drying columns. ${ }^{1} \mathrm{MeCN}$ was then degassed by sparging with $\mathrm{N}_{2}$. PrCN was degassed by sparging with $\mathrm{N}_{2}$. Oven-dried potassium carbonate was added to $\mathrm{CDCl}_{3}$ to remove trace acid. Potassium carbonate for crosscoupling was dried in the oven overnight then stored in a desiccator. $4 \AA$ Molecular sieves were purchased and heated under vacuum then crushed and stored in the desiccator. Amino acid methyl esters were obtained via a procedure reported in the literature unless otherwise noted. ${ }^{2}$ Proton nuclear magnetic resonance $\left({ }^{1} \mathrm{H}\right.$ NMR) spectra, carbon nuclear magnetic resonance $\left({ }^{13} \mathrm{C} \mathrm{NMR}\right)$ spectra, and fluorine nuclear magnetic resonance spectra ( ${ }^{19} \mathrm{~F}$ NMR) were recorded on both 400 $\mathrm{MHz}$ and $600 \mathrm{MHz}$ spectrometers. Chemical shifts for protons are reported in parts per million downfield from tetramethylsilane and are referenced to residual protium in the NMR solvent $\left(\mathrm{CHCl}_{3}=\delta\right.$ 7.26). Chemical shifts for carbon are reported in parts per million downfield from tetramethylsilane and are referenced to the carbon resonances of the solvent $\left(\mathrm{CDCl}_{3}=\delta 77.16\right)$. Chemical shifts for fluorine were externally referenced to $\mathrm{CFCl}_{3}$ in $\mathrm{CDCl}_{3}\left(\mathrm{CFCl}_{3}=\delta 0\right)$. Data are represented as follows: chemical shift, multiplicity $(\mathrm{br}=$ broad, $\mathrm{s}=\operatorname{singlet}, \mathrm{d}=$ doublet, $\mathrm{t}=$ triplet, $\mathrm{q}=$ quartet, $\mathrm{p}=$ pentet, $\mathrm{m}=$ multiplet, $\mathrm{dd}=$ doublet of doublets, $\mathrm{ddd}=$ doublet of doublets of doublets, $\mathrm{dt}=$ doublet of triplets, $\mathrm{dq}=$ doublet of quartets, $\mathrm{dp}=$ doublet of pentets, $\mathrm{tt}=$ triplet of triplets, $\mathrm{td}=$ triplet of doublets, $\mathrm{h}=$ heptet$)$, coupling constants in Hertz $(\mathrm{Hz})$, integration. Infrared (IR) spectra were obtained using FTIR spectrophotometers with material loaded onto a KBr plate. The mass spectral data were obtained at the University of Delaware mass spectrometry facility. Melting points were taken on a Thomas-Hoover Uni-Melt Capillary Melting Point Apparatus. 


\section{Optimization Studies}<smiles></smiles>

$3 b$

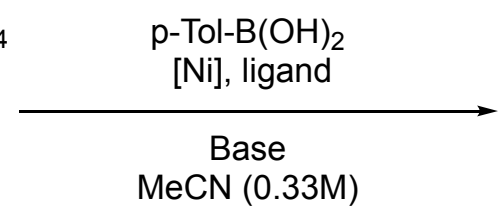

$\operatorname{MeCN}(0.33 \mathrm{M})$

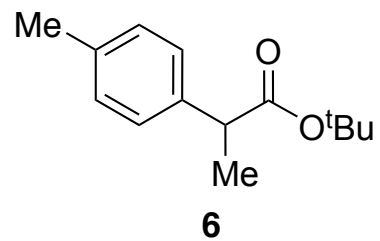

6

General Optimization Procedure. In a $\mathrm{N}_{2}$-atmosphere glovebox: nickel, ligand, base, boronic acid, and pyridinium substrate $\mathbf{3 b}(52.3 \mathrm{mg}, 0.10 \mathrm{mmol}, 1.0$ equiv) were weighed into an oven-dried 1-dram vial fitted with a stir bar. $\mathrm{MeCN}(0.3 \mathrm{~mL}, 0.33 \mathrm{M})$ was added, and the vial was capped with a Teflon-lined cap and removed from the glovebox. The mixture was stirred at $70{ }^{\circ} \mathrm{C}$ for $24 \mathrm{~h}$, unless otherwise stated. The mixture was then diluted with $\mathrm{Et}_{2} \mathrm{O}(3 \mathrm{~mL})$ and filtered through a plug of silica gel, which was rinsed with $\mathrm{Et}_{2} \mathrm{O}(10 \mathrm{~mL})$. The filtrate was concentrated. 1,3,5,-Trimethoxybenzene (internal standard) was added. $\mathrm{CDCl}_{3}$ was added, and the yield was determined by ${ }^{1} \mathrm{H}$ NMR analysis. Changes to this general procedure are noted in the tables below.

\begin{tabular}{|c|c|c|c|c|c|}
\hline entry & {$[\mathrm{Ni}]$} & ligand & $p$-Tol-BX 2 & base & yield $(\%)^{a}$ \\
\hline 1 & $\mathrm{Ni}(\operatorname{cod})_{2}$ & 1,10-phen & $p$-Tol-B(OH $)_{2}$ & $\mathrm{NaOMe}$ & 10 \\
\hline 2 & $\mathrm{Ni}(\operatorname{cod})_{2}$ & 1,10-phen & $p$-Tol-B $(\mathrm{OH})_{2}$ & $\mathrm{~K}_{3} \mathrm{PO}_{4}$ & 6 \\
\hline 3 & $\mathrm{Ni}(\operatorname{cod})_{2}$ & 1,10-phen & $p$-Tol-B $(\mathrm{OH})_{2}$ & $\mathrm{~K}_{2} \mathrm{CO}_{3}$ & 69 \\
\hline $4^{b}$ & $\mathrm{Ni}(\operatorname{cod})_{2}$ & 1,10-phen & $p$-Tol-B $(\mathrm{OH})_{2}$ & $\mathrm{~K}_{2} \mathrm{CO}_{3}$ & 77 \\
\hline $5^{b}$ & $\mathrm{NiCl}_{2} \cdot \mathrm{DME}$ & 1,10-phen & $p$-Tol-B $(\mathrm{OH})_{2}$ & $\mathrm{~K}_{2} \mathrm{CO}_{3}$ & 75 \\
\hline $6^{b, c}$ & $\mathrm{NiCl}_{2} \cdot \mathrm{DME}$ & 1,10-phen & $p$-Tol- $\mathrm{B}(\mathrm{OH})_{2}$ & $\mathrm{~K}_{2} \mathrm{CO}_{3}$ & 88 \\
\hline $7^{b, c}$ & $\mathrm{NiCl}_{2} \cdot \mathrm{DME}$ & 4,4'-dmbpy & $p$-Tol-B $(\mathrm{OH})_{2}$ & $\mathrm{~K}_{2} \mathrm{CO}_{3}$ & $>99$ \\
\hline $8^{c, d}$ & $\mathrm{NiCl}_{2} \cdot \mathrm{DME}$ & 4,4'-dmbpy & $(p-\text { Tol-BO })_{3}$ & $\mathrm{~K}_{2} \mathrm{CO}_{3}$ & $>99$ \\
\hline $9^{d, e}$ & $\mathrm{NiCl}_{2} \cdot \mathrm{DME}$ & 4,4'-dmbpy & $(p-\text { Tol-BO })_{3}$ & $\mathrm{~K}_{2} \mathrm{CO}_{3}$ & 71 \\
\hline $10^{c, d, f}$ & $\mathrm{NiCl}_{2} \cdot \mathrm{DME}$ & 4,4'-dmbpy & $(p-\mathrm{Tol}-\mathrm{BO})_{3}$ & $\mathrm{~K}_{2} \mathrm{CO}_{3}$ & No Rxn \\
\hline $11^{c, d}$ & --- & 4,4'-dmbpy & $(p-\text { Tol-BO })_{3}$ & $\mathrm{~K}_{2} \mathrm{CO}_{3}$ & No Rxn \\
\hline
\end{tabular}

Conditions: pyridinium salt 3 b $(0.10 \mathrm{mmol}, 1.0$ equiv), [Ni] (10 mol \%), ligand (12\%), boronic acid (1.5 equiv), base (1.7 equiv), $\operatorname{MeCN}(0.33 \mathrm{M}), 70{ }^{\circ} \mathrm{C}, 24 \mathrm{~h} .{ }^{a}$ Determined by ${ }^{1} \mathrm{H}$ NMR using 1,3,5-trimethoxybenzene as internal standard. ${ }^{b}$ Boronic acid (2.5 equiv), base (2.8 equiv). ${ }^{c}$ [Ni] (5 mol \%), ligand (6\%), $4 \AA \mathrm{MS} .{ }^{d}$ Boroxine ( 0.8 equiv), base $\left(2.8\right.$ equiv). ${ }^{e}[\mathrm{Ni}](2 \mathrm{~mol} \%)$, ligand (3\%). ${ }^{f}$ Room temperature. 


\section{Investigation of Metal Source}<smiles></smiles>

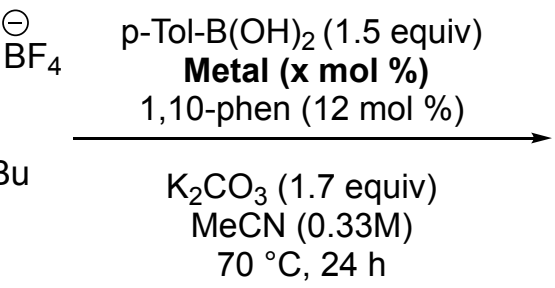<smiles>CCCCOC(=O)C(C)c1ccc(C)cc1</smiles>

\begin{tabular}{ccc} 
entry & Metal source $(\mathrm{mol} \%)$ & Yield $^{a}$ \\
\hline 1 & $\mathrm{Pd}_{2}(\mathrm{dba})_{3}(5)$ & 0 \\
2 & $\mathrm{NiCl}_{2} \cdot \mathrm{DME}(10)$ & 75 \\
3 & $\mathrm{Ni}(\mathrm{OAc})_{2} \cdot 4 \mathrm{H}_{2} \mathrm{O}(10)$ & 0 \\
4 & $\mathrm{Ni}(\mathrm{cod})_{2}(10)$ & 69
\end{tabular}

${ }^{a}$ Determined by ${ }^{1} \mathrm{H}$ NMR using 1,3,5-trimethoxybenzene as internal standard.

\section{Investigation of Ligand}<smiles></smiles>

3b entry
$\ominus_{\mathrm{BF}} \quad \mathrm{p}-\mathrm{Tol}-\mathrm{B}(\mathrm{OH})_{2}(1.5$ equiv $)$ $\mathrm{F}_{4} \quad \mathrm{Ni}(\mathrm{cod})_{2}(10 \mathrm{~mol} \%)$ ligand $(12 \mathrm{~mol} \%)$

$\mathrm{K}_{2} \mathrm{CO}_{3}$ (1.7 equiv) THF (0.33M) $70^{\circ} \mathrm{C}, 24 \mathrm{~h}$ ligand 1,10-phenanthroline 2,2'-bipyridine

3 1,1'-Bis(diphenylphosphino)ferrocene (dppf) 0

4 Tricyclohexylphosphine ( $\left.\mathrm{PCy}_{3}\right)$

0

5

XPhos

${ }^{a}$ Determined by ${ }^{1} \mathrm{H}$ NMR using 1,3,5-trimethoxybenzene as internal standard. 


\section{Investigation of Base}

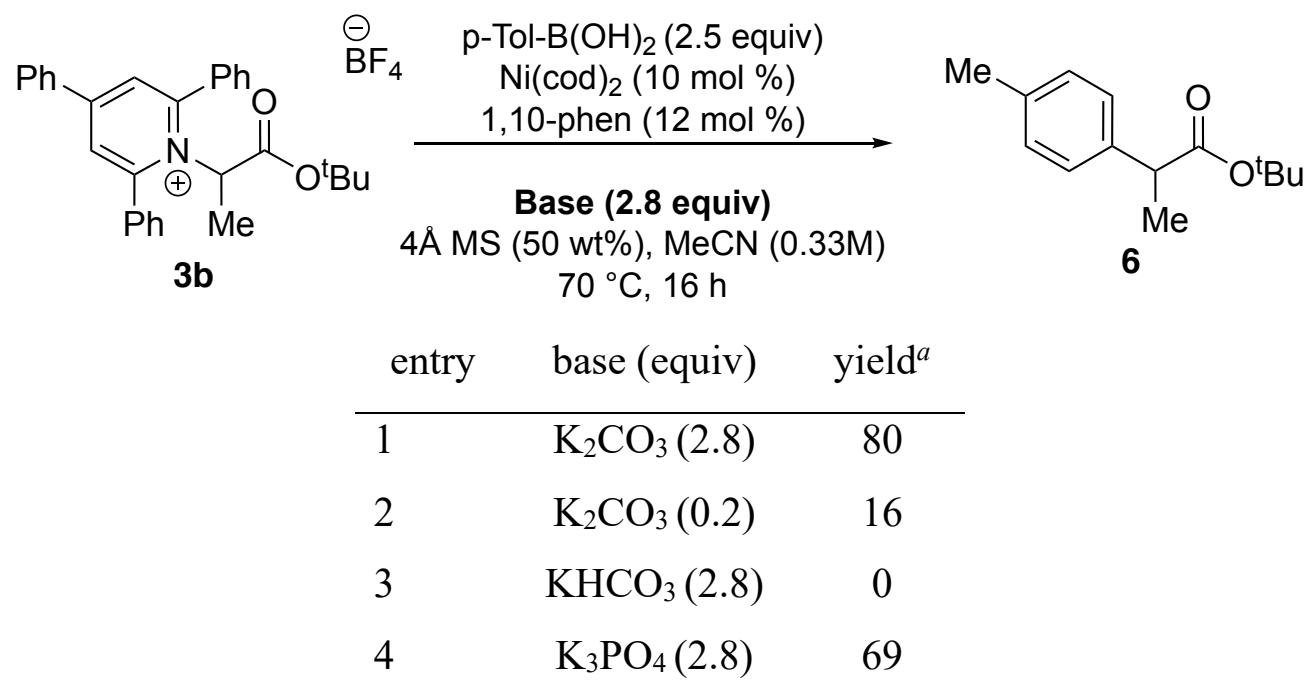

${ }^{a}$ Determined by ${ }^{1} \mathrm{H}$ NMR using 1,3,5-trimethoxybenzene as internal standard.

\section{Investigation of Solvent}<smiles>CCCCOC(=O)C(C)N1C(c2ccccc2)=CC(c2ccccc2)=CC1Pc1ccccc1</smiles>

$3 b$

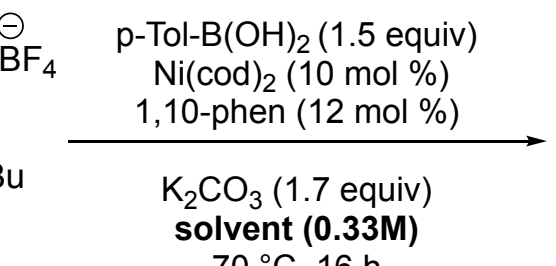

$70{ }^{\circ} \mathrm{C}, 16 \mathrm{~h}$<smiles>CCCOC(=O)C(C)c1ccc(C)cc1</smiles>

6

\begin{tabular}{ccc} 
entry & solvent & yield $^{a}$ \\
\hline 1 & THF & 15 \\
2 & MeCN & 69 \\
3 & Toluene & 5 \\
4 & $\mathrm{CHCl}_{3}$ & 4
\end{tabular}

${ }^{a}$ Determined by ${ }^{1} \mathrm{H}$ NMR using 1,3,5-trimethoxybenzene as internal standard. 


\section{Investigation of Solvents for Sterically Hindered Substrates}<smiles></smiles>

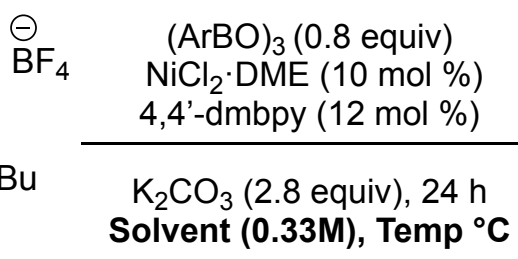

3d<smiles>COC(=O)C(c1cccc(OC)c1)C(C)C</smiles>

\begin{tabular}{cccl} 
entry & solvent & temp $\left({ }^{\circ} \mathrm{C}\right)$ & Yield $^{a}$ \\
\hline 1 & MeCN & 70 & 29 \\
2 & MeCN & 80 & 53 \\
3 & MeCN & 90 & 48 \\
4 & PrCN & 70 & 21 \\
5 & PrCN & 80 & 71 \\
6 & PrCN & 90 & 43
\end{tabular}

${ }^{a}$ Determined by ${ }^{1} \mathrm{H}$ NMR using 1,3,5-trimethoxybenzene as internal standard.

\section{Limitation in Pyridinium Salt}<smiles></smiles>
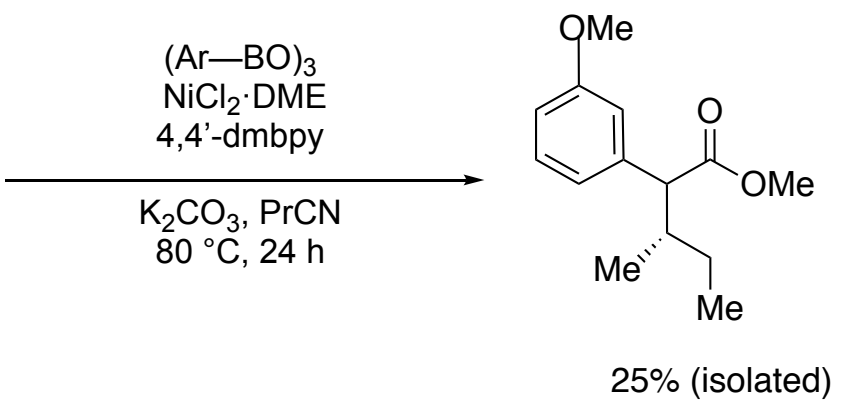

Pyridinium of isoleucine formed in moderate yield however when used in the cross-coupling the product was difficult to isolate. The cross-coupling isolated yield for isoleucine was $25 \%$ using conditions similar to valine. ( $\mathrm{PrCN}$ as the solvent at $80^{\circ} \mathrm{C}$ ) 


\section{Limitations in Boroxine Scope}

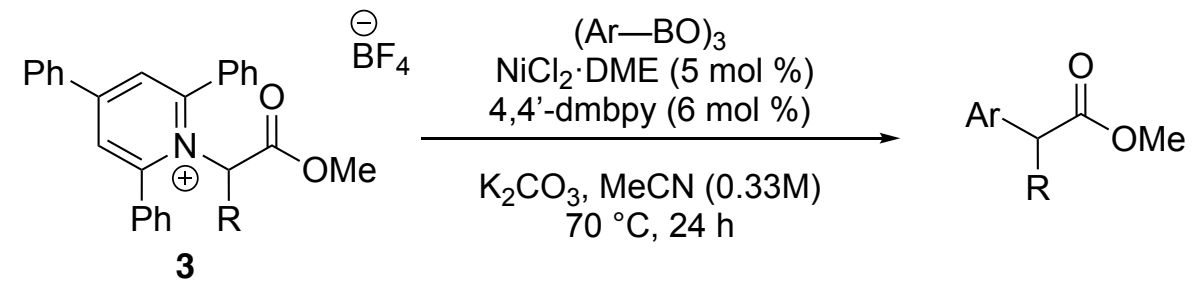

heteroatoms:<smiles>COC(=O)C(C)c1ccc2c(ccn2C)c1</smiles><smiles>COC(=O)C(C)c1ccc2occc2c1</smiles>

$16 \%$ (NMR) $29 \%(N M R)$

ortho substitution:<smiles>COC(=O)C(C)c1cccnc1F</smiles><smiles>COC(=O)C(C)c1ccccc1C</smiles>
$12 \%$ (NMR)

\section{Suzuki-Miyaura Cross-Couplings of Amino Acid Pyridinium Salts}

\section{General Procedure A: Cross-Coupling with Aryl Boroxines}

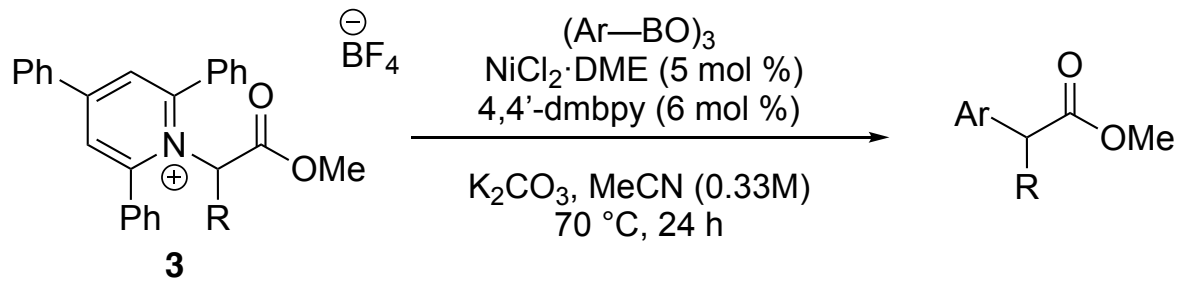

To an oven-dried, $10-\mathrm{mL}$ round-bottomed flask fitted with a stir bar was added $\mathrm{NiCl}_{2} \cdot \mathrm{DME}$ (11 mg, $0.050 \mathrm{mmol}, 5 \mathrm{~mol} \%$ ), 4,4'-dimethyl-2,2'-dipyridyl (4,4'-dmbpy, $11 \mathrm{mg}, 0.060 \mathrm{mmol}, 6$ mol \%), $\mathrm{K}_{2} \mathrm{CO}_{3}$ (387 mg, $2.8 \mathrm{mmol}, 2.8$ equiv), aryl boroxine (0.80 mmol, 0.80 equiv), and pyridinium salt 3 (1.0 mmol, 1.0 equiv). The flask was fitted with a rubber septum, sealed with parafilm, and then purged with $\mathrm{N}_{2}$ for $20 \mathrm{~min}$. The vent needle was removed, and acetonitrile (3 $\mathrm{mL}$ ) was added. The $\mathrm{N}_{2}$ inlet was removed, and the mixture was stirred at $70{ }^{\circ} \mathrm{C}$ for $24 \mathrm{~h}$. The mixture was allowed to cool to room temperature. Then $\mathrm{Et}_{2} \mathrm{O}(3 \mathrm{~mL})$ was added. The mixture was filtered through silica gel with $\mathrm{Et}_{2} \mathrm{O}(3 \mathrm{~mL})$ and concentrated. The cross-coupled product was then purified via silica gel chromatography. 


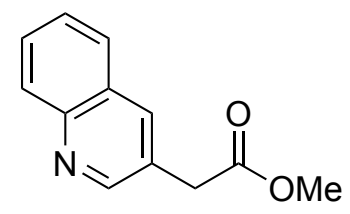

Methyl 2-(quinolin-3-yl)acetate (7). Prepared via General Procedure A using pyridinium salt 3c. The crude mixture was purified by silica gel chromatography ( $50 \% \mathrm{Et}_{2} \mathrm{O} /$ hexanes) to give 7 (run 1: $140 \mathrm{mg}, 70 \%$; run 2: $143 \mathrm{mg}, 70 \%)$ as a yellow oil: ${ }^{1} \mathrm{H} \mathrm{NMR}\left(600 \mathrm{MHz}, \mathrm{CDCl}_{3}\right) \delta 8.84(\mathrm{~d}, J=$ $2.3 \mathrm{~Hz}, 1 \mathrm{H}), 8.12-8.06(\mathrm{~m}, 2 \mathrm{H}), 7.80(\mathrm{dd}, J=8.2,1.4 \mathrm{~Hz}, 1 \mathrm{H}), 7.70(\mathrm{ddd}, J=8.4,6.8,1.5 \mathrm{~Hz}$, $1 \mathrm{H}), 7.55$ (ddd, $J=8.1,6.8,1.2 \mathrm{~Hz}, 1 \mathrm{H}), 3.83$ (s, 2H), 3.73 (s, $3 \mathrm{H}) ;{ }^{13} \mathrm{C} \mathrm{NMR}\left(151 \mathrm{MHz}, \mathrm{CDCl}_{3}\right.$ ) $\delta 171.3,151.8,147.4,136.0,129.5,129.4,128.0,127.7,127.0,127.0,52.5,38.6$. The spectral data matches that previously reported in the literature. ${ }^{3}$

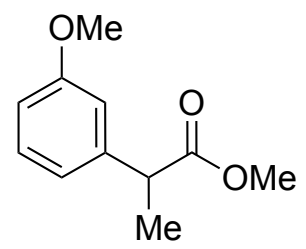

Methyl 2-(3-methoxyphenyl)propanoate (8). Prepared via General Procedure A using pyridinium salt 3a. The crude mixture was purified by silica gel chromatography $(50 \%$ $\mathrm{Et}_{2} \mathrm{O} /$ hexanes) to give 8 (run 1: $184 \mathrm{mg}, 88 \%$; run 2: $167 \mathrm{mg}, 86 \%$ ) as a light yellow oil: ${ }^{1} \mathrm{H}$ NMR $\left(400 \mathrm{MHz}, \mathrm{CDCl}_{3}\right) \delta 7.23(\mathrm{~d}, J=7.9 \mathrm{~Hz}, 1 \mathrm{H}), 6.90-6.78(\mathrm{~m}, 3 \mathrm{H}), 3.81(\mathrm{~s}, 3 \mathrm{H}), 3.74-3.68(\mathrm{~m}$, 1H), $3.66(\mathrm{~s}, 3 \mathrm{H}), 1.49$ (d, $J=7.2 \mathrm{~Hz}, 3 \mathrm{H}) ;{ }^{13} \mathrm{C} \mathrm{NMR}\left(101 \mathrm{MHz}, \mathrm{CDCl}_{3}\right) \delta$ 175.0, 159.9, 142.2, $129.8,120.0,113.4,112.6,55.4,52.2,45.6,18.7$. The spectral data matches that previously reported in the literature. ${ }^{4}$

Product 8 was also prepared on a $5.0-\mathrm{mmol}$ scale: To an oven-dried, $100-\mathrm{mL}$ roundbottomed flask fitted with a stir bar was added $\mathrm{NiCl}_{2}$. DME (55 mg, $0.25 \mathrm{mmol}, 5 \mathrm{~mol} \%$ ), 4,4'dimethyl-2,2'-dipyridyl (4,4'-dmbpy, $55 \mathrm{mg}, 0.30 \mathrm{mmol}, 6 \mathrm{~mol} \%$ ), $\mathrm{K}_{2} \mathrm{CO}_{3}(1.93 \mathrm{~g}, 14 \mathrm{mmol}, 2.8$ equiv), aryl boroxine ( $1.61 \mathrm{~g}, 4 \mathrm{mmol}, 0.80$ equiv), and pyridinium salt 3a ( $2.40 \mathrm{~g}, 5.0 \mathrm{mmol}, 1.0$ equiv). The flask was fitted with a rubber septum, sealed with parafilm, and then purged with $\mathrm{N}_{2}$ for $20 \mathrm{~min}$. The vent needle was removed, and acetonitrile $(15 \mathrm{~mL})$ was added. The $\mathrm{N}_{2}$ inlet was removed, and the mixture was stirred at $70{ }^{\circ} \mathrm{C}$ for $24 \mathrm{~h}$. The mixture was allowed to cool to room temperature. Then $\mathrm{Et}_{2} \mathrm{O}(15 \mathrm{~mL})$ was added. The mixture was filtered through silica gel with $\mathrm{Et}_{2} \mathrm{O}$ $(15 \mathrm{~mL})$ and concentrated. The crude material was then purified via silica gel chromatography 
(50\% $\mathrm{Et}_{2} \mathrm{O} /$ hexanes) to give 8 (run 1: $613 \mathrm{mg}, 63 \%$ ) as a yellow oil. The spectra matched that above.

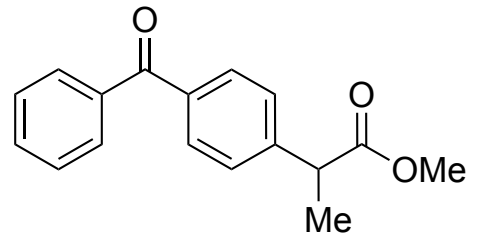

Methyl 2-(4-benzoylphenyl)propanoate (9). Prepared via General Procedure A using pyridinium salt 3a. The crude mixture was purified by silica gel chromatography $\left(10 \% \mathrm{Et}_{2} \mathrm{O} /\right.$ hexanes $)$ to give 9 (run 1: $224 \mathrm{mg}, 77 \%$; run 2: $223 \mathrm{mg}, 76 \%$ ) as a yellow solid (mp 69-71 $\left.{ }^{\circ} \mathrm{C}\right):{ }^{1} \mathrm{H} \mathrm{NMR}(600 \mathrm{MHz}$, $\left.\mathrm{CDCl}_{3}\right) \delta 7.82-7.75(\mathrm{~m}, 4 \mathrm{H}), 7.61-7.56(\mathrm{~m}, 1 \mathrm{H}), 7.50-7.45(\mathrm{~m}, 2 \mathrm{H}), 7.44-7.39(\mathrm{~m}, 2 \mathrm{H}), 3.82$ (q, $J=7.2 \mathrm{~Hz}, 1 \mathrm{H}), 3.69$ (s, 3H), 1.55 (q, $J=7.2 \mathrm{~Hz}, 3 \mathrm{H}) ;{ }^{13} \mathrm{C}$ NMR $\left(101 \mathrm{MHz}, \mathrm{CDCl}_{3}\right) \delta 196.3$, $174.4,145.2,137.6,136.5,132.5,130.6,130.0,128.3,127.6,52.3,45.5,18.5$. The spectral data matches that previously reported in the literature..$^{5}$<smiles>COC(=O)C(C)c1ccc(N(c2ccccc2)c2ccccc2)cc1</smiles>

Methyl 2-(4-(diphenylamino)phenyl)propanoate (10). Prepared via General Procedure A using pyridinium salt 3a. The crude mixture was purified by silica gel chromatography $(1 \rightarrow 10 \%$ EtOAc/hexanes) to give 10 (run 1: $193 \mathrm{mg}, 59 \%$; run 2: $200 \mathrm{mg}, 61 \%$ ) as a yellow oil: ${ }^{1} \mathrm{H}$ NMR $\left(400 \mathrm{MHz}, \mathrm{CDCl}_{3}\right) \delta 7.28-7.23(\mathrm{~m}, 4 \mathrm{H}), 7.20-7.15(\mathrm{~m}, 2 \mathrm{H}), 7.13-7.08(\mathrm{~m}, 4 \mathrm{H}), 7.07-6.99$ (m, 4H), $3.71(\mathrm{~m}, 4 \mathrm{H}), 1.51(\mathrm{~d}, J=7.2 \mathrm{~Hz}, 3 \mathrm{H}) ;{ }^{13} \mathrm{C}$ NMR (101 MHz, $\left.\mathrm{CDCl}_{3}\right) \delta 175.4,147.8$, 146.9, 134.6, 129.3, 128.3, 124.4, 124.0, 122.9, 52.2, 44.9, 18.7; FTIR (neat) 3033, 2924, 1737 , 1589, 1491, 1278, 1165, 754, $696 \mathrm{~cm}^{-1}$; HRMS (ESI+) $[\mathrm{M}+\mathrm{H}]^{+}$calculated for $\mathrm{C}_{22} \mathrm{H}_{22} \mathrm{NO}_{2}$ : $332.1650,332.1647$.<smiles>COC(=O)C(C)c1cnc(F)c(C)c1</smiles>

Methyl 2-(6-fluoro-5-methylpyridin-3-yl)propanoate (11). Prepared via General Procedure A using pyridinium salt 3a. The crude mixture was purified by silica gel chromatography $(2 \rightarrow 40 \%$ EtOAc/hexanes) to give 11 (run 1: $124 \mathrm{mg}, 63 \%$; run 2: $130 \mathrm{mg}, 66 \%$ ) as a yellow oil: ${ }^{1} \mathrm{H}$ NMR 
$\left(600 \mathrm{MHz}, \mathrm{CDCl}_{3}\right) \delta 7.92-7.91(\mathrm{~m}, 1 \mathrm{H}), 7.56-7.54(\mathrm{~m}, 1 \mathrm{H}), 3.71(\mathrm{q}, J=7.2 \mathrm{~Hz}, 1 \mathrm{H}), 3.67(\mathrm{~s}$, $3 \mathrm{H}), 2.27(\mathrm{dt}, J=1.4,0.8 \mathrm{~Hz}, 3 \mathrm{H}), 1.49(\mathrm{~d}, J=7.2 \mathrm{~Hz}, 3 \mathrm{H}) ;{ }^{13} \mathrm{C} \mathrm{NMR}\left(151 \mathrm{MHz}, \mathrm{CDCl}_{3}\right) \delta 174.3$, $161.7\left(\mathrm{~d}, J_{\mathrm{C}-\mathrm{F}}=238.6 \mathrm{~Hz}\right), 143.8\left(\mathrm{~d}, J_{\mathrm{C}-\mathrm{F}}=15.1 \mathrm{~Hz}\right), 140.6\left(\mathrm{~d}, J_{\mathrm{C}-\mathrm{F}}=6.04 \mathrm{~Hz}\right), 133.8\left(\mathrm{~d}, J_{\mathrm{C}-\mathrm{F}}=4.53\right.$ $\mathrm{Hz}), 119.7\left(\mathrm{~d}, J_{\mathrm{C}-\mathrm{F}}=31.71 \mathrm{~Hz}\right), 52.4,42.2,18.7,14.6\left(\mathrm{~d}, J_{\mathrm{C}-\mathrm{F}}=1.51 \mathrm{~Hz}\right) ;{ }^{19} \mathrm{~F}$ NMR $(565 \mathrm{MHz}$, $\left.\mathrm{CDCl}_{3}\right) \delta$-74.6; FTIR (neat) 2929, 1738, 1419, 1054, $749 \mathrm{~cm}^{-1}$; HRMS (ESI+) $[\mathrm{M}+\mathrm{H}]^{+}$calculated for $\mathrm{C}_{10} \mathrm{H}_{13} \mathrm{FNO}_{2}$ : 198.0930, found 198.0925.

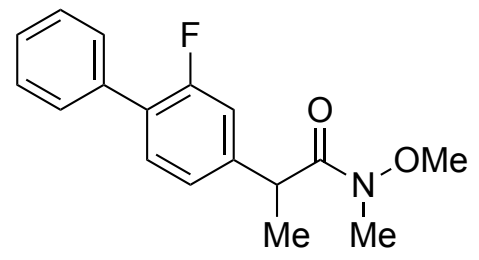

2-(2-Fluoro-[1,1'-biphenyl]-4-yl)- $N$-methoxy- $N$-methylpropanamide (12). Prepared via General Procedure A using pyridinium salt 3d. The crude mixture was purified by silica gel chromatography (25\% $\mathrm{Et}_{2} \mathrm{O} /$ hexanes) to give 12 (run 1: $242 \mathrm{mg}, 87 \%$ ) as a yellow oil: ${ }^{1} \mathrm{H}$ NMR $\left(600 \mathrm{MHz}, \mathrm{CDCl}_{3}\right) \delta 7.53(\mathrm{dt}, J=8.1,1.5 \mathrm{~Hz}, 2 \mathrm{H}), 7.46-7.41(\mathrm{~m}, 2 \mathrm{H}), 7.39-7.33(\mathrm{~m}, 2 \mathrm{H}), 7.18$ $-7.11(\mathrm{~m}, 2 \mathrm{H}), 4.19$ (br s, 1H), 3.55 (s, 3H), 3.20 (s, 3H), 1.49 (d, J=7.1 Hz, 3H); ${ }^{13} \mathrm{C}$ NMR (101

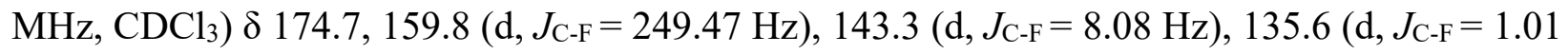
$\mathrm{Hz}), 130.8\left(\mathrm{~d}, J_{\mathrm{C}-\mathrm{F}}=4.04 \mathrm{~Hz}\right), 129.0\left(\mathrm{~d}, J_{\mathrm{C}-\mathrm{F}}=3.03 \mathrm{~Hz}\right), 128.5,127.7,127.5\left(\mathrm{~d}, J_{\mathrm{C}-\mathrm{F}}=13.13 \mathrm{~Hz}\right)$, $123.7\left(\mathrm{~d}, J_{\mathrm{C}-\mathrm{F}}=3.03 \mathrm{~Hz}\right), 115.4\left(\mathrm{~d}, J_{\mathrm{C}-\mathrm{F}}=23.23 \mathrm{~Hz}\right), 61.4,41.3,31.5\left(\mathrm{~d}, J_{\mathrm{C}-\mathrm{F}}=212.1 \mathrm{~Hz}\right), 19.5 ;{ }^{19} \mathrm{~F}$ NMR (565 MHz, $\left.\mathrm{CDCl}_{3}\right) \delta-117.8$; FTIR (neat) 2973, 1664, 1484, 1268, 991, 767, $699 \mathrm{~cm}^{-1}$; HRMS (ESI+) $[\mathrm{M}+\mathrm{H}]^{+}$calculated for $\mathrm{C}_{17} \mathrm{H}_{19} \mathrm{FNO}_{2}$ : 288.1400, found 288.1396.

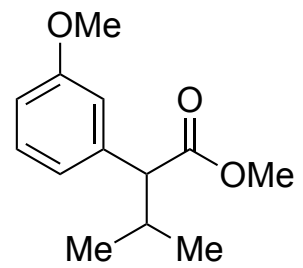

Methyl 2-(3-methoxyphenyl)-3-methylbutanoate (13). Prepared via General Procedure A using pyridinium salt 3e, except that butyronitrile was used in place of $\mathrm{MeCN}$ and the reaction mixture was heated at $80{ }^{\circ} \mathrm{C}$. The crude mixture was purified by silica gel chromatography (1\% EtOAc/Toluene) to give 13 (run 1: $93.1 \mathrm{mg}, 42 \%$; run 2: $99.8 \mathrm{mg}, 45 \%$ ) as a yellow oil: ${ }^{1} \mathrm{H}$ NMR $\left(600 \mathrm{MHz}, \mathrm{CDCl}_{3}\right) \delta 7.22(\mathrm{t}, J=7.9 \mathrm{~Hz}, 1 \mathrm{H}), 6.92-6.88(\mathrm{~m}, 2 \mathrm{H}), 6.80(\mathrm{dd}, J=8.1,2.6 \mathrm{~Hz}, 1 \mathrm{H})$, $3.80(\mathrm{~s}, 3 \mathrm{H}), 3.65(\mathrm{~s}, 3 \mathrm{H}), 3.12(\mathrm{~d}, J=10.6 \mathrm{~Hz}, 1 \mathrm{H}), 2.32(\mathrm{dp}, J=10.6,6.6 \mathrm{~Hz}, 1 \mathrm{H}), 1.02(\mathrm{~d}, J=$ 
$6.5 \mathrm{~Hz}, 3 \mathrm{H}), 0.71(\mathrm{~d}, J=6.7 \mathrm{~Hz}, 3 \mathrm{H}) ;{ }^{13} \mathrm{C} \mathrm{NMR}\left(151 \mathrm{MHz}, \mathrm{CDCl}_{3}\right) \delta 174.4,159.8,140.0,129.5$, 121.1, 114.3, 112.7, 60.2, 55.3, 51.9, 32.1, 21.6, 20.6; FTIR (neat) 2926, 1735, 1289, $668 \mathrm{~cm}^{-1}$; HRMS (ESI+) $[\mathrm{M}+\mathrm{H}]^{+}$calculated for $\mathrm{C}_{13} \mathrm{H}_{19} \mathrm{O}_{3}: 223.1334$, found 223.1330 .

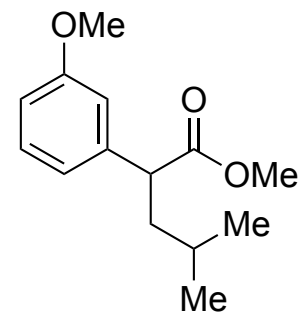

Methyl 2-(3-methoxyphenyl)-4-methylpentanoate (14). Prepared via General Procedure A using pyridinium salt 3f, except that butyronitrile was used in place of $\mathrm{MeCN}$ and the reaction mixture was heated at $80^{\circ} \mathrm{C}$. The crude mixture was purified by silica gel chromatography $(0 \rightarrow 5 \%$ EtOAc/Toluene) to give 14 (run 1: $984 \mathrm{mg}, 42 \%$ ) as a yellow oil: ${ }^{1} \mathrm{H} \mathrm{NMR}\left(600 \mathrm{MHz}, \mathrm{CDCl}_{3}\right) \delta$ $7.23(\mathrm{t}, J=7.9 \mathrm{~Hz}, 1 \mathrm{H}), 6.91-6.77(\mathrm{~m}, 3 \mathrm{H}), 3.80(\mathrm{~s}, 3 \mathrm{H}), 3.65(\mathrm{~s}, 3 \mathrm{H}), 3.63(\mathrm{t}, J=7.9 \mathrm{~Hz}, 1 \mathrm{H})$, $1.94(\mathrm{dt}, J=13.6,7.7 \mathrm{~Hz}, 1 \mathrm{H}), 1.67(\mathrm{dt}, J=13.9,7.1 \mathrm{~Hz}, 1 \mathrm{H}), 1.45(\mathrm{dq}, J=13.5,6.7 \mathrm{~Hz}, 1 \mathrm{H})$, $0.92-0.87(\mathrm{~m}, 6 \mathrm{H}) ;{ }^{13} \mathrm{C} \mathrm{NMR}\left(151 \mathrm{MHz}, \mathrm{CDCl}_{3}\right) \delta 174.7,159.9,141.0,129.7,120.5,113.9$, 112.6, 55.3, 52.1, 49.7, 42.6, 26.0, 22.8, 22.4; FTIR (neat) 2956, 1737, 1259, 1165, 1050, $776 \mathrm{~cm}^{-}$ 1; HRMS (ESI+) $[\mathrm{M}+\mathrm{H}]^{+}$calculated for $\mathrm{C}_{14} \mathrm{H}_{21} \mathrm{O}_{3}: 237.1490$, found 237.1485 .<smiles>COC(=O)C(Cc1ccccc1)c1ccc(OC)nc1</smiles>

Methyl 2-(6-methoxypyridin-3-yl)-3-phenylpropanoate (15). Prepared via General Procedure A using pyridinium salt $3 \mathrm{~g}$. The crude mixture was purified by silica gel chromatography $(5 \rightarrow 20 \%$ EtOAc/hexanes) to give 15 (run 1: $176 \mathrm{mg}, 65 \%$; run 2: $190 \mathrm{mg}, 70 \%$ ) as a dark yellow oil: ${ }^{1} \mathrm{H}$ $\operatorname{NMR}\left(600 \mathrm{MHz}, \mathrm{CDCl}_{3}\right) \delta 7.99(\mathrm{~d}, J=2.5 \mathrm{~Hz}, 1 \mathrm{H}), 7.57$ (dd, $\left.J=8.6,2.5 \mathrm{~Hz}, 1 \mathrm{H}\right), 7.23$ (dd, $J=$ 8.1, 6.7 Hz, 2H), 7.18 (t, $J=7.3 \mathrm{~Hz}, 1 \mathrm{H}), 7.11-7.06(\mathrm{~m}, 2 \mathrm{H}), 6.70(\mathrm{~d}, J=8.6 \mathrm{~Hz}, 1 \mathrm{H}), 3.91$ (s, $3 \mathrm{H}), 3.80(\mathrm{t}, J=7.8 \mathrm{~Hz}, 1 \mathrm{H}), 3.62(\mathrm{~s}, 3 \mathrm{H}), 3.38$ (dd, $J=13.8,8.2 \mathrm{~Hz}, 1 \mathrm{H}), 2.99$ (dd, $J=13.8,7.5$ $\mathrm{Hz}, 1 \mathrm{H}) ;{ }^{13} \mathrm{C} \mathrm{NMR}\left(151 \mathrm{MHz}, \mathrm{CDCl}_{3}\right) \delta 173.7,163.7,146.5,138.6,138.1,129.0,128.6,127.0$, 126.7, 111.0, 53.6, 52.3, 50.3, 39.8; FTIR (neat) 3059, 2950, 1734, 1617, 1248, 1030, 762, 700 $\mathrm{cm}^{-1}$; HRMS (ESI+) $[\mathrm{M}+\mathrm{H}]^{+}$calculated for $\mathrm{C}_{16} \mathrm{H}_{18} \mathrm{NO}_{3}: 272.1286$, found 272.1291 . 


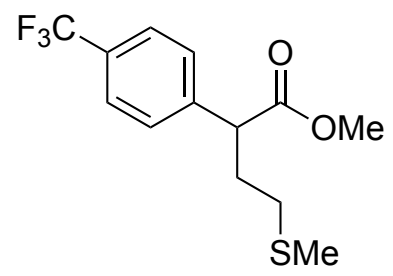

Methyl 4-(methylthio)-2-(4-(trifluoromethyl)phenyl)butanoate (16). Prepared via General Procedure A using pyridinium salt $\mathbf{3 h}$. The crude mixture was purified by silica gel chromatography (2\% EtOAc/hexanes) to give 16 (run 1: $189 \mathrm{mg}, 64 \%$; run 2: $210 \mathrm{mg}, 71 \%$ ) as a yellow oil: ${ }^{1} \mathrm{H}$ NMR $\left(600 \mathrm{MHz}, \mathrm{CDCl}_{3}\right) \delta 7.62-7.57(\mathrm{~m}, 2 \mathrm{H}), 7.43(\mathrm{~d}, J=8.0 \mathrm{~Hz}, 2 \mathrm{H}), 3.87(\mathrm{t}, J$ $=7.2 \mathrm{~Hz}, 1 \mathrm{H}), 3.68(\mathrm{~s}, 3 \mathrm{H}), 2.49-2.34(\mathrm{~m}, 3 \mathrm{H}), 2.10-2.01(\mathrm{~m}, 4 \mathrm{H}) ;{ }^{13} \mathrm{C} \mathrm{NMR}\left(151 \mathrm{MHz}, \mathrm{CDCl}_{3}\right)$ $\delta 173.5,142.5,129.9\left(\mathrm{q}, J_{\mathrm{C}-\mathrm{F}}=31.7 \mathrm{~Hz}\right), 128.6,125.8\left(\mathrm{q}, J_{\mathrm{C}-\mathrm{F}}=4.5 \mathrm{~Hz}\right), 124.1\left(\mathrm{q}, J_{\mathrm{C}-\mathrm{F}}=271.8 \mathrm{~Hz}\right)$, 52.4, 49.9, 32.4, 31.8, 15.4; ${ }^{19} \mathrm{~F} \mathrm{NMR}\left(565 \mathrm{MHz}, \mathrm{CDCl}_{3}\right) \delta$-62.6; FTIR (neat) 2954, 2361, 1736, 1327, 1167, $837 \mathrm{~cm}^{-1}$; HRMS $(\mathrm{ESI}+)[\mathrm{M}+\mathrm{H}]^{+}$calculated for $\mathrm{C}_{13} \mathrm{H}_{16} \mathrm{~F}_{3} \mathrm{O}_{2} \mathrm{~S}: 293.0823$, found 293.0814.

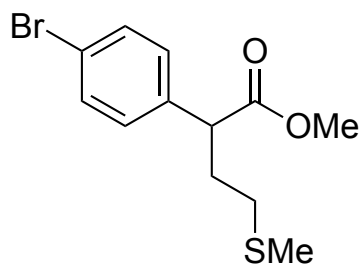

Methyl 2-(4-bromophenyl)-4-(methylthio)butanoate (17). Prepared via General Procedure A using pyridinium salt $\mathbf{3 h}$. The crude mixture was purified by silica gel chromatography $(5 \rightarrow 40 \%$ $\mathrm{Et}_{2} \mathrm{O} /$ hexanes) to give 17 (run 1: $233 \mathrm{mg}, 77 \%$; run 2: $224 \mathrm{mg}, 74 \%$ ) as a pale yellow oil: ${ }^{1} \mathrm{H}$ NMR $\left(600 \mathrm{MHz}, \mathrm{CDCl}_{3}\right) \delta 7.47-7.42(\mathrm{~m}, 2 \mathrm{H}), 7.20-7.14(\mathrm{~m}, 2 \mathrm{H}), 3.75(\mathrm{t}, J=7.3 \mathrm{~Hz}, 1 \mathrm{H}), 3.65(\mathrm{~s}$, $3 \mathrm{H}), 2.46-2.30(\mathrm{~m}, 3 \mathrm{H}), 2.06(\mathrm{~s}, 3 \mathrm{H}), 2.03-1.96(\mathrm{~m}, 1 \mathrm{H}) ;{ }^{13} \mathrm{C} \mathrm{NMR}\left(101 \mathrm{MHz}, \mathrm{CDCl}_{3}\right) \delta 173.7$, 137.4, 132.0, 129.9, 121.6, 52.4, 49.4, 32.3, 31.8, 15.4; FTIR (neat) 2950, 1735, 1488, 1167, 1011, $822 \mathrm{~cm}^{-1}$; HRMS (ESI+) $[\mathrm{M}+\mathrm{H}]^{+}$calculated for $\mathrm{C}_{12} \mathrm{H}_{16} \mathrm{BrO}_{2} \mathrm{~S}: 303.0054$, found 303.0049 .<smiles>COC(=O)C(Cc1c[nH]c2ccccc12)c1cccc(OC)c1</smiles>

Methyl 3-(1H-indol-3-yl)-2-(3-methoxyphenyl)propanoate (18). Prepared via General Procedure A using pyridinium salt 3i. The crude mixture was purified by silica gel chromatography 
( $5 \rightarrow 25 \%$ EtOAc/hexanes) to give 18 (run 1: $189 \mathrm{mg}, 59 \%$; run 2: $190 \mathrm{mg}, 60 \%$ ) as a dark orange oil: ${ }^{1} \mathrm{H}$ NMR (600 MHz, $\left.\mathrm{CDCl}_{3}\right) \delta 7.93(\mathrm{~s}, 1 \mathrm{H}), 7.60(\mathrm{~d}, J=7.9,0.9 \mathrm{~Hz}, 1 \mathrm{H}), 7.33$ (dt, $J=8.1,0.9$ $\mathrm{Hz}, 1 \mathrm{H}), 7.24(\mathrm{~d}, J=8.0 \mathrm{~Hz}, 1 \mathrm{H}), 7.21-7.17(\mathrm{~m}, 1 \mathrm{H}), 7.14-7.11(\mathrm{~m}, 1 \mathrm{H}), 6.96(\mathrm{dt}, J=7.6,1.2$ $\mathrm{Hz}, 1 \mathrm{H}), 6.93(\mathrm{t}, J=2.1 \mathrm{~Hz}, 1 \mathrm{H}), 6.88(\mathrm{~d}, J=2.3 \mathrm{~Hz}, 1 \mathrm{H}), 6.82$ (ddd, $J=8.3,2.6,0.9 \mathrm{~Hz}, 1 \mathrm{H})$, $3.98(\mathrm{dd}, J=9.0,6.3 \mathrm{~Hz}, 1 \mathrm{H}), 3.79$ (s, $3 \mathrm{H}), 3.61$ (s, $3 \mathrm{H}), 3.60-3.57$ (m, 1H), 3.18 (ddd, $J=14.6$, 6.3, $0.9 \mathrm{~Hz}, 1 \mathrm{H}) ;{ }^{13} \mathrm{C} \mathrm{NMR}\left(151 \mathrm{MHz}, \mathrm{CDCl}_{3}\right) \delta 174.3,159.9,140.9,136.2,129.7,127.5,122.5$, 122.1, 120.5, 119.5, 118.8, 113.8, 113.5, 112.9, 111.2, 55.4, 52.6, 52.1, 29.5; FTIR (neat) 3417, 1733, 1457, 1260, 1163, $743 \mathrm{~cm}^{-1}$; HRMS (ESI+) $[\mathrm{M}+\mathrm{H}]^{+}$calculated for $\mathrm{C}_{19} \mathrm{H}_{20} \mathrm{NO}_{3}: 310.1443$, found 310.1433 .<smiles>COC(=O)C(Cc1ccc(O)cc1)c1cccc(OC)c1</smiles>

Methyl 3-(4-hydroxyphenyl)-2-(3-methoxyphenyl)propanoate (19). Prepared via General Procedure A using pyridinium salt $\mathbf{3 j}$. The crude mixture was purified by silica gel chromatography ( $5 \rightarrow 50 \%$ EtOAc/hexanes) to give 19 (run 1: $251 \mathrm{mg}, 87 \%$; run 2: $238 \mathrm{mg}, 82 \%$ ) as a yellow/orange oil: ${ }^{1} \mathrm{H} \mathrm{NMR}\left(600 \mathrm{MHz}, \mathrm{CDCl}_{3}\right) \delta 7.22(\mathrm{t}, J=7.9 \mathrm{~Hz}, 1 \mathrm{H}), 7.01-6.95(\mathrm{~m}, 2 \mathrm{H})$, $6.89-6.78(\mathrm{~m}, 3 \mathrm{H}), 6.72-6.68(\mathrm{~m}, 2 \mathrm{H}), 4.71(\mathrm{~s}, 1 \mathrm{H}), 3.79(\mathrm{~s}, 3 \mathrm{H}), 3.77(\mathrm{dd}, J=8.9,6.6 \mathrm{~Hz}, 1 \mathrm{H})$, $3.61(\mathrm{~s}, 3 \mathrm{H}), 3.32(\mathrm{dd}, J=13.9,8.9 \mathrm{~Hz}, 1 \mathrm{H}), 2.95(\mathrm{dd}, J=13.8,6.6 \mathrm{~Hz}, 1 \mathrm{H}) ;{ }^{13} \mathrm{C} \mathrm{NMR}(151 \mathrm{MHz}$, $\left.\mathrm{CDCl}_{3}\right) \delta 174.1,159.9,154.3,140.2,131.2,130.2,129.7,120.5,115.4,113.8,113.0,55.4,54.0$, 52.2, 39.0; FTIR (neat) 3410, 2952, 1734, 1516, 1263, $695 \mathrm{~cm}^{-1}$; HRMS (ESI+) $[\mathrm{M}+\mathrm{H}]^{+}$calculated for $\mathrm{C}_{17} \mathrm{H}_{19} \mathrm{O}_{4}$ : 287.1283, found 287.1274.<smiles>COC(=O)C(Cc1ccc(O)c(O)c1)c1cccc(OC)c1</smiles>

Methyl 3-(3,4-dihydroxyphenyl)-2-(3-methoxyphenyl)propanoate (20). Prepared via General Procedure A using pyridinium salt 3k. The crude mixture was purified by silica gel chromatography ( $10 \rightarrow 80 \%$ EtOAc/hexanes) to give 20 (run 1: $115 \mathrm{mg}, 41 \%$; run 2: $307 \mathrm{mg}, 43 \%$ ) 
as a dark yellow oil: ${ }^{1} \mathrm{H} \mathrm{NMR}\left(400 \mathrm{MHz}, \mathrm{CDCl}_{3}\right) \delta 7.22(\mathrm{t}, J=7.9 \mathrm{~Hz}, 1 \mathrm{H}), 6.90-6.78(\mathrm{~m}, 3 \mathrm{H})$, $6.74-6.55(\mathrm{~m}, 3 \mathrm{H}), 5.17(\mathrm{~d}, J=1.3 \mathrm{~Hz}, 1 \mathrm{H}), 5.09$ (d, $J=1.2 \mathrm{~Hz}, 1 \mathrm{H}), 3.79$ (s, 3H), $3.78-3.73$ (m, 1H), $3.61(\mathrm{~s}, 3 \mathrm{H}), 3.28(\mathrm{dd}, J=13.8,9.1 \mathrm{~Hz}, 1 \mathrm{H}), 2.90(\mathrm{dd}, J=13.8,6.4 \mathrm{~Hz}, 1 \mathrm{H}) ;{ }^{13} \mathrm{C} \mathrm{NMR}$ $\left(101 \mathrm{MHz}, \mathrm{CDCl}_{3}\right) \delta 174.0,159.8,143.4,142.1,140.2,132.2,129.8,121.6,120.4,116.1,115.4$, 113.7, 112.9, 55.4, 53.9, 52.3, 39.2; FTIR (neat) 3396, 2953, 1711, 1601, 1520, 1437, 1261, 1163, $781 \mathrm{~cm}^{-1}$; HRMS (ESI+) $[\mathrm{M}+\mathrm{H}]^{+}$calculated for $\mathrm{C}_{17} \mathrm{H}_{19} \mathrm{O}_{5}: 303.0054$, found 303.1233 .

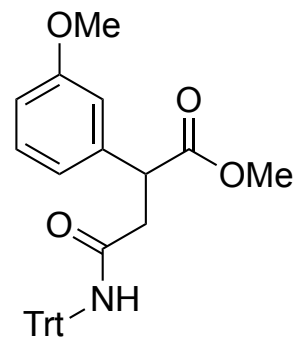

Methyl 2-(3-methoxyphenyl)-4-oxo-4-(tritylamino)butanoate (21). Prepared via General Procedure A using pyridinium salt 31. The crude mixture was purified by silica gel chromatography $(1 \rightarrow 20 \%$ EtOAc/hexanes) to give 21 (run 1: $244 \mathrm{mg}, 51 \%$ ) as a light yellow solid (mp 140-142 $\left.{ }^{\circ} \mathrm{C}\right):{ }^{1} \mathrm{H}$ NMR $\left(600 \mathrm{MHz}, \mathrm{CDCl}_{3}\right) \delta 7.27(\mathrm{~d}, J=1.9 \mathrm{~Hz}, 1 \mathrm{H}), 7.26-7.21(\mathrm{~m}, 8 \mathrm{H}), 7.14-7.09$ (m, $6 \mathrm{H}), 6.88-6.80(\mathrm{~m}, 3 \mathrm{H}), 6.67(\mathrm{~s}, 1 \mathrm{H}), 4.14(\mathrm{dd}, J=9.3,6.0 \mathrm{~Hz}, 1 \mathrm{H}), 3.78(\mathrm{~s}, 3 \mathrm{H}), 3.64(\mathrm{~s}, 3 \mathrm{H})$, $3.09(\mathrm{dd}, J=15.0,9.3 \mathrm{~Hz}, 1 \mathrm{H}), 2.63(\mathrm{dd}, J=14.9,6.0 \mathrm{~Hz}, 1 \mathrm{H}) ;{ }^{13} \mathrm{C} \mathrm{NMR}\left(151 \mathrm{MHz}, \mathrm{CDCl}_{3}\right) \delta$ 174.0, 169.3 , 160.1 144.6, 139.7, 130.0, 128.8, 128.8, 127.1, 120.2, 113.7, 113.4, 70.7, 55.4, 52.5, 47.6, 41.5; FTIR (neat) 3319, 3057, 1734, 1491, 1262, 1037, $699 \mathrm{~cm}^{-1}$; HRMS (ESI+) [M+H] calculated for $\mathrm{C}_{31} \mathrm{H}_{30} \mathrm{NO}_{4}: 480.2175$, found 480.2152 .

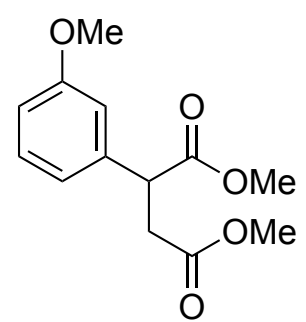

Dimethyl 2-(3-methoxyphenyl)succinate (22). Prepared via General Procedure A, but on half the normal scale, using pyridinium salt $\mathbf{3 m}(0.5 \mathrm{mmol})$. The crude mixture was purified by silica gel chromatography (10\% $\mathrm{Et}_{2} \mathrm{O} /$ hexanes) to give 22 (run 1: $87 \mathrm{mg}, 68 \%$; run 2: $74 \mathrm{mg}, 57 \%$ ) as a yellow oil: ${ }^{1} \mathrm{H}$ NMR $\left(600 \mathrm{MHz}, \mathrm{CDCl}_{3}\right) \delta 7.25$ - $7.22(\mathrm{~m}, 1 \mathrm{H}), 6.86(\mathrm{dt}, J=7.6,1.2 \mathrm{~Hz}, 1 \mathrm{H}), 6.82$ (dd, $J=4.4,1.9 \mathrm{~Hz}, 2 \mathrm{H}), 4.06$ (dd, $J=10.2,5.1 \mathrm{~Hz}, 1 \mathrm{H}), 3.79$ (s, 3H), 3.68 (d, $J=2.7 \mathrm{~Hz}, 6 \mathrm{H})$, $3.20(\mathrm{dd}, J=17.0,10.2 \mathrm{~Hz}, 1 \mathrm{H}), 2.66(\mathrm{dd}, J=17.0,5.1 \mathrm{~Hz}, 1 \mathrm{H}) ;{ }^{13} \mathrm{C} \mathrm{NMR}\left(101 \mathrm{MHz}, \mathrm{CDCl}_{3}\right) \delta$ 
$173.5,172.2,160.0,139.2,130.1,120.1,113.6,113.2,55.4,52.6,52.1,47.2,37.7$. The spectral data matches that previously reported in the literature. ${ }^{6}$

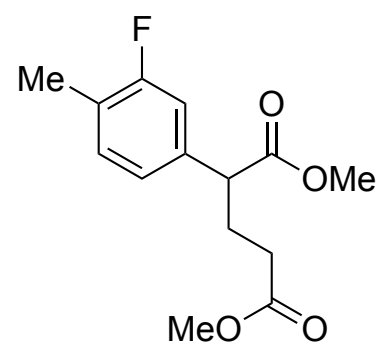

Dimethyl 2-(3-fluoro-4-methylphenyl)pentanedioate (23). Prepared via General Procedure A using pyridinium salt $\mathbf{3 n}$. The crude mixture was purified by silica gel chromatography (5\% EtOAc/hexanes) to give 23 (run 1: $223 \mathrm{mg}, 83 \%$; run 2: $210 \mathrm{mg}, 78 \%$ ) as a yellow oil: ${ }^{1} \mathrm{H}$ NMR $\left(400 \mathrm{MHz}, \mathrm{CDCl}_{3}\right) \delta 7.14-7.10(\mathrm{~m}, 1 \mathrm{H}), 6.96-6.93(\mathrm{~m}, 2 \mathrm{H}), 3.66(\mathrm{~d}, J=2.9 \mathrm{~Hz}, 6 \mathrm{H}), 3.58(\mathrm{t}, J$ $=7.6 \mathrm{~Hz}, 1 \mathrm{H}), 2.39-2.29(\mathrm{~m}, 1 \mathrm{H}), 2.27(\mathrm{~d}, J=6.8 \mathrm{~Hz}, 2 \mathrm{H}), 2.24(\mathrm{~s}, 3 \mathrm{H}), 2.13-2.03(\mathrm{~m}, 1 \mathrm{H})$; ${ }^{13} \mathrm{C} \mathrm{NMR}\left(101 \mathrm{MHz}, \mathrm{CDCl}_{3}\right) \delta 173.7,173.3,161.4\left(\mathrm{~d}, J_{\mathrm{C}-\mathrm{F}}=245.4 \mathrm{~Hz}\right), 137.8\left(\mathrm{~d}, J_{\mathrm{C}-\mathrm{F}}=7.1 \mathrm{~Hz}\right)$, $131.8\left(\mathrm{~d}, J_{\mathrm{C}-\mathrm{F}}=5.1 \mathrm{~Hz}\right), 124.2\left(\mathrm{~d}, J_{\mathrm{C}-\mathrm{F}}=17.2 \mathrm{~Hz}\right), 123.5\left(\mathrm{~d}, J_{\mathrm{C}-\mathrm{F}}=4.0 \mathrm{~Hz}\right), 114.6\left(\mathrm{~d}, J_{\mathrm{C}-\mathrm{F}}=23.2\right.$ $\mathrm{Hz}), 52.4,51.8,49.9\left(\mathrm{~d}, J_{\mathrm{C}-\mathrm{F}}=2.0 \mathrm{~Hz}\right), 31.6,28.3,14.4\left(\mathrm{~d}, J_{\mathrm{C}-\mathrm{F}}=4.0 \mathrm{~Hz}\right) ;{ }^{19} \mathrm{~F} \mathrm{NMR}(376 \mathrm{MHz}$, $\left.\mathrm{CDCl}_{3}\right) \delta-116.74$; FTIR (neat) 2953, 1738, 1436, $1253 \mathrm{~cm}^{-1}$; HRMS (ESI+) $[\mathrm{M}+\mathrm{H}]^{+}$calculated for $\mathrm{C}_{14} \mathrm{H}_{18} \mathrm{FO}_{4}: 269.1189$, found 269.1182 .

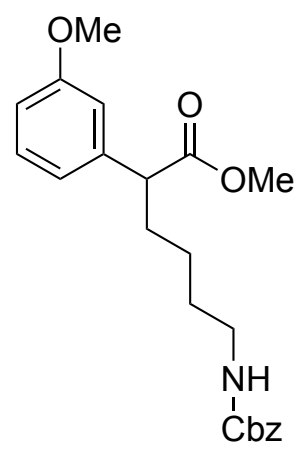

Methyl 6-(((benzyloxy)carbonyl)amino)-2-(3-methoxyphenyl)hexanoate (24). Prepared via General Procedure A using pyridinium salt 3o. The crude mixture was purified by silica gel chromatography $\left(20 \rightarrow 100 \% \mathrm{MeCN} / \mathrm{H}_{2} \mathrm{O}\right)$ to give 24 (run 1: $222 \mathrm{mg}, 57 \%$ ) as a brown oil: ${ }^{1} \mathrm{H}$ NMR $\left(400 \mathrm{MHz}, \mathrm{CDCl}_{3}\right) \delta 7.39-7.28(\mathrm{~m}, 5 \mathrm{H}), 7.23(\mathrm{t}, J=7.9 \mathrm{~Hz}, 1 \mathrm{H}), 6.88-6.77(\mathrm{~m}, 3 \mathrm{H}), 5.08(\mathrm{~s}$, 2H), $4.79(\mathrm{~m}, 1 \mathrm{H}), 3.79(\mathrm{~s}, 3 \mathrm{H}), 3.64(\mathrm{~s}, 3 \mathrm{H}), 3.49$ (t, $J=7.7 \mathrm{~Hz}, 1 \mathrm{H}), 3.15$ (q, $J=6.8 \mathrm{~Hz}, 2 \mathrm{H})$, $2.12-1.98(\mathrm{~m}, 1 \mathrm{H}), 1.83-1.69(\mathrm{~m}, 1 \mathrm{H}), 1.50(\mathrm{td}, J=9.4,8.8,4.9 \mathrm{~Hz}, 2 \mathrm{H}), 1.26(\mathrm{tt}, J=6.8,4.4$ $\mathrm{Hz}, 2 \mathrm{H}) ;{ }^{13} \mathrm{C} \mathrm{NMR}\left(101 \mathrm{MHz}, \mathrm{CDCl}_{3}\right) \delta 174.3,159.8,156.4,140.5,136.7,129.7,128.6,128.20$, 
128.18 120.3, 113.7, 112.6, 66.7, 55.3, 52.1, 51.5, 40.9, 33.1, 29.8, 24.8; FTIR (neat) 3350, 2947, $1729,1528,1256,1161,1047,776,694 \mathrm{~cm}^{-1}$; HRMS (ESI+) $[\mathrm{M}+\mathrm{H}]^{+}$calculated for $\mathrm{C}_{22} \mathrm{H}_{28} \mathrm{NO}_{5}$ : 386.1967 , found 386.1965 .<smiles>COC(=O)C(CCCNC(=N)NS(=O)(=O)c1c(C)c(C)c2c(c1C)OC(C)(C)O2)c1cccc(OC)c1</smiles>

Methyl 2-(3-methoxyphenyl)-5-(3-((2,2,4,6,7-pentamethyl-2,3-dihydrobenzofuran-5yl)sulfonyl)guanidino)pentanoate (25). Prepared via General Procedure A using pyridinium salt 3p. The crude mixture was purified by silica gel chromatography $(10 \rightarrow 100 \%$ EtOAc/hexanes) to give 25 (run 1: $265 \mathrm{mg}, 63 \%$ ) as a white solid (mp 60-62 $\left.{ }^{\circ} \mathrm{C}\right):{ }^{1} \mathrm{H}$ NMR $\left(400 \mathrm{MHz}, \mathrm{CDCl}_{3}\right) \delta 7.24$ $-7.17(\mathrm{~m}, 1 \mathrm{H}), 6.84-6.76(\mathrm{~m}, 3 \mathrm{H}), 5.69(\mathrm{~d}, J=99.7 \mathrm{~Hz}, 2 \mathrm{H}), 3.79(\mathrm{~s}, 3 \mathrm{H}), 3.64(\mathrm{~s}, 3 \mathrm{H}), 3.48(\mathrm{t}$, $J=7.7 \mathrm{~Hz}, 1 \mathrm{H}), 3.12$ (q, $J=6.5 \mathrm{~Hz}, 2 \mathrm{H}), 2.94$ (s, 2H), 2.55 (s, 3H), 2.49 (s, 3H), 2.08 (s, 3H), 2.04 $(\mathrm{m}, J=8.0,4.2 \mathrm{~Hz}, 1 \mathrm{H}), 1.82-1.70(\mathrm{~m}, 1 \mathrm{H}), 1.59(\mathrm{~s}, 1 \mathrm{H}), 1.46(\mathrm{~m}, 8 \mathrm{H}) ;{ }^{13} \mathrm{C}$ NMR $(101 \mathrm{MHz}$, $\left.\mathrm{CDCl}_{3}\right) \delta 174.2,159.9,158.9,155.8,140.1,138.6,132.8,132.5,129.9,124.7,120.3,117.6,113.7$, 112.8, 86.5, 55.4, 52.3, 51.2, 43.3, 41.3, 30.5, 28.8, 27.3, 19.4, 18.0, 12.6; FTIR (neat) 3445, 3339, $1734,1558,1258,668 \mathrm{~cm}^{-1}$; HRMS (ESI+) $[\mathrm{M}+\mathrm{H}]^{+}$calculated for $\mathrm{C}_{27} \mathrm{H}_{38} \mathrm{~N}_{3} \mathrm{O}_{6} \mathrm{~S}: 532.2481$, found 532.2481 .

\section{Sequential One-pot Cross-Coupling of Alanine Methyl Ester}

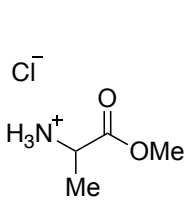

$4 a$

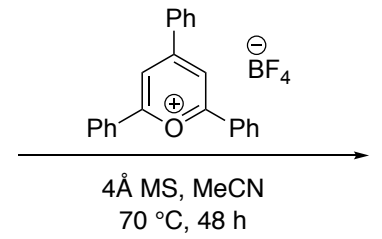

$70{ }^{\circ} \mathrm{C}, 48 \mathrm{~h}$

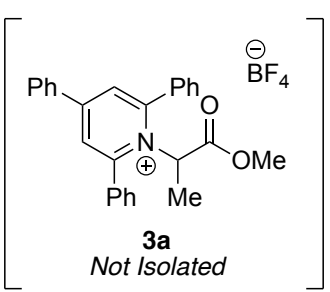

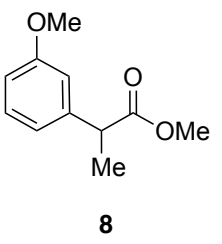

Product 8 was formed via a procedure adapted from General Procedure A. To an ovendried, 10-mL round-bottomed flask was added alanine methyl ester hydrochloride salt (139 mg, $1.0 \mathrm{mmol}, 1.0$ equiv), 2,4,6-triphenylpyrillium tetrafluoroborate (396 mg, $1.0 \mathrm{mmol}, 1.0$ equiv), 
and powdered, activated $4 \AA$ molecular sieves $(500 \mathrm{mg}, 500 \mathrm{mg} / \mathrm{mmol})$. The flask was fitted with a septum and acetonitrile $(6.0 \mathrm{~mL})$ was added. The resulting mixture was stirred at $70{ }^{\circ} \mathrm{C}$ for $48 \mathrm{~h}$. The mixture was allowed to cool to room temperature then $\mathrm{NiCl}_{2} \cdot \mathrm{DME}(11 \mathrm{mg}, 0.050 \mathrm{mmol}, 5$ mol \%), 4,4'-dimethyl-2,2'-dipyridyl (4,4'-dmbpy, $11 \mathrm{mg}, 0.060 \mathrm{mmol}, 6 \mathrm{~mol} \%), \mathrm{K}_{2} \mathrm{CO}_{3}(387$ $\mathrm{mg}, 2.8 \mathrm{mmol}, 2.8$ equiv), and aryl boroxine ( $0.80 \mathrm{mmol}, 0.80$ equiv) were added. The flask was again fitted with a septum, and the mixture was stirred at $70{ }^{\circ} \mathrm{C}$ for $24 \mathrm{~h}$. The mixture was allowed to cool to room temperature, and then filtered through a small pad of Celite. The filter cake was washed with diethyl ether $(4 \times 25 \mathrm{~mL})$. Then the filtrate was concentrated. The crude mixture was purified by silica gel chromatography ( $5 \%$ ether/hexanes) to give $\mathbf{8}$ (70 $\mathrm{mg}, 36 \%$ ).

\section{Preparation of Pyridium Salts}

\section{Optimization of Pyridinium Salt Synthesis}

In our initial work with alkyl amines with unactived alkyl groups, ${ }^{7}$ the pyridinium salts could be readily synthesized by heating the amine and 2,4,6-triphenylpyrylium in refluxing EtOH. However, when this method was applied to $\alpha$-amino esters, poor to moderate yields were obtained. Higher yields were achieved when the amino ester and pyrylium salt were mixed at room temperature in $\mathrm{CH}_{2} \mathrm{Cl}_{2}$ in the presence of $\mathrm{Et}_{3} \mathrm{~N}$, followed by acidification with AcOH. ${ }^{8}$ In some cases, particularly for more hindered substrates, diketone $\mathbf{S - 1}$, a byproduct of hydrolysis of the iminium ion intermediate, was observed. The use of $4 \AA ̊$ molecular sieves decreased formation of diketone $\mathbf{S - 1}$, and in some cases (alanine and tryptophan esters and Weinreb amide) the pyridinium salts could be used without purification via chromatography or recrystallization. Small amounts of this diketone $(<10 \%)$ did not appear to affect the subsequent cross-coupling; however, significant quantities did diminish the yield. 


\section{Investigation of Desiccant on Pyridinium Salt Formation}

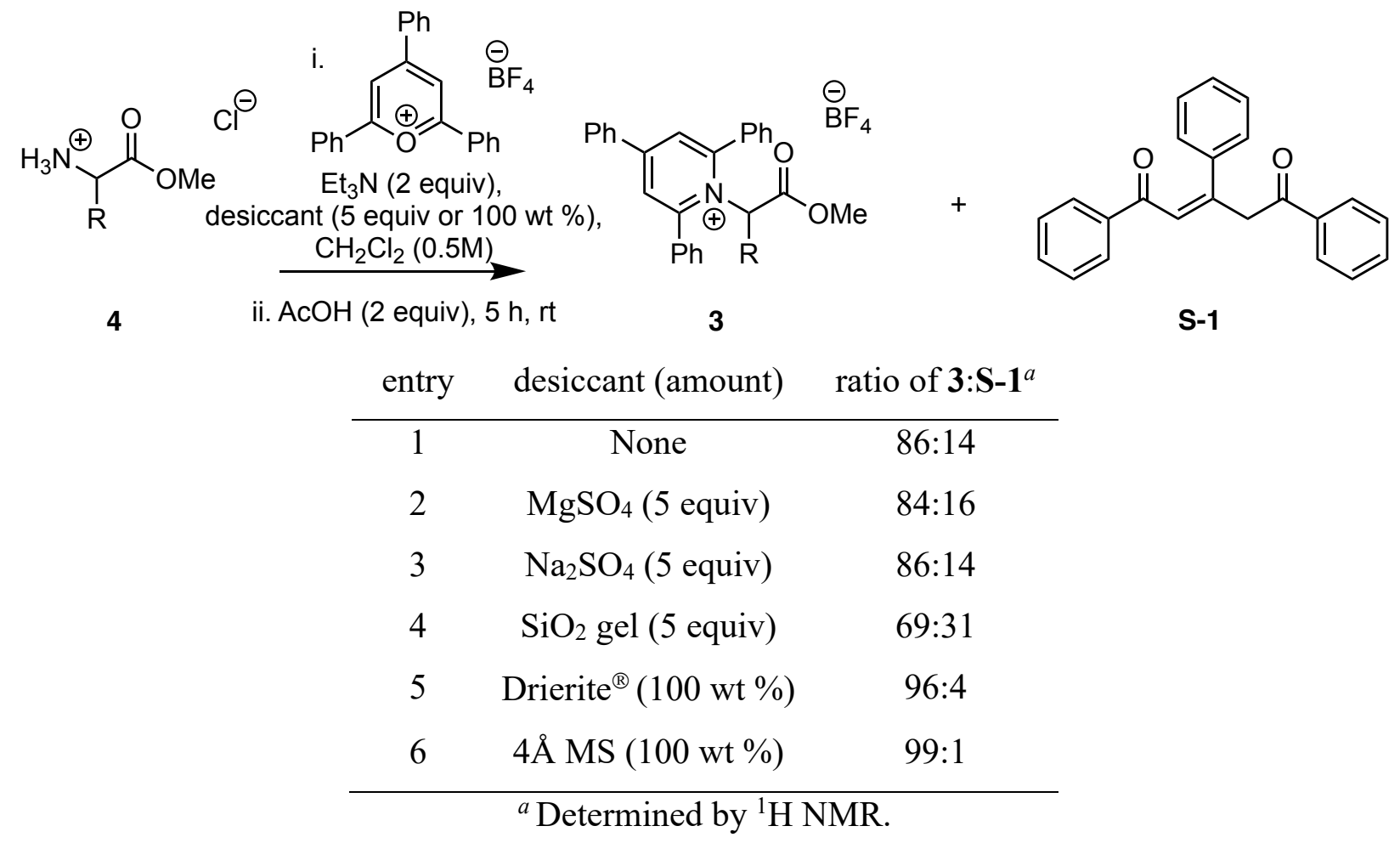

Investigation of the Effect of Diketone Byproduct S-1 on the Cross-Coupling<smiles>[R]C(C(=O)OC)N1C(P)=CC(c2ccccc2)=CC1Pc1ccccc1</smiles>

3

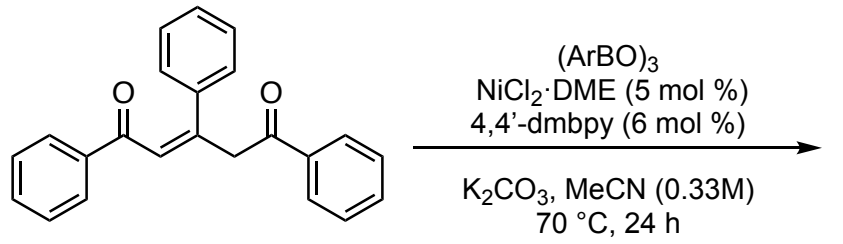

S-1

\begin{tabular}{|c|c|c|c|}
\hline entry & $\mathrm{R}$ & pyridinium salt (3): diketone $(\mathbf{S}-\mathbf{1})$ & yield $^{a}$ \\
\hline 1 & & $20: 1$ & 75 \\
\hline 2 & & $>20: 1^{b}$ & 77 \\
\hline 3 & & $6: 1$ & 22 \\
\hline 4 & & $>20: 1^{b}$ & 60 \\
\hline
\end{tabular}

${ }^{a}$ Determined by ${ }^{1} \mathrm{H}$ NMR using 1,3,5-trimethoxybenzene as internal standard. ${ }^{b}$ Pyridinium salt purified by silica gel chromatography. 


\section{Limitations in Pyridinium Synthesis}

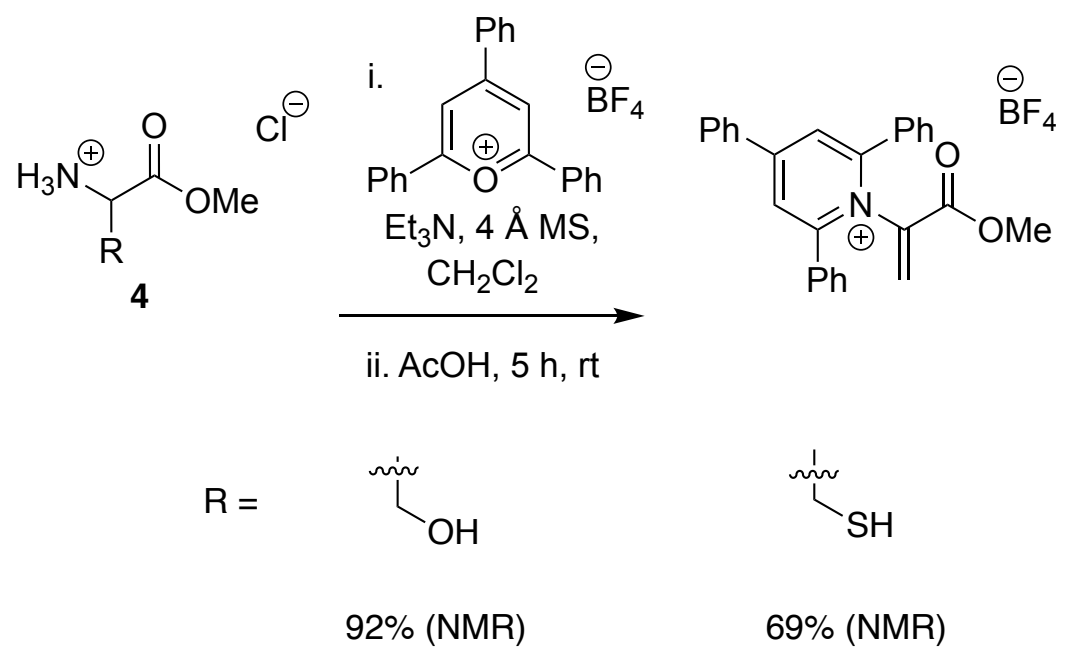

Under the conditions typically used to form the pyridinium salt, serine and cysteine methyl esters formed a known dehydroalanine elimination product ${ }^{9}$ instead of the desired pyridinium salt.<smiles>COC(=O)C([18O])Cc1cnc[nH]1</smiles>

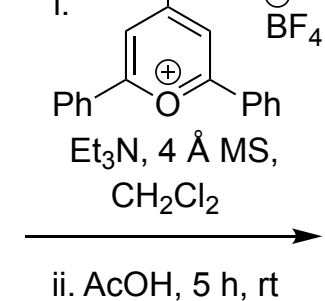
ii. $\mathrm{AcOH}, 5 \mathrm{~h}, \mathrm{rt}$<smiles></smiles>

$0 \%(\mathrm{NMR})$<smiles>O=C(/C=C(/CC(=O)c1ccccc1)c1ccccc1)c1ccccc1</smiles>

$16 \%(N M R)$<smiles>COC(=O)Cn1cnc(CC([NH3+])C(=O)OC)c1</smiles>

Unprotected histidine did not form the pyridinium salt after numerous tries. Protecting histidine was tried but then the methyl ester could not be formed. 

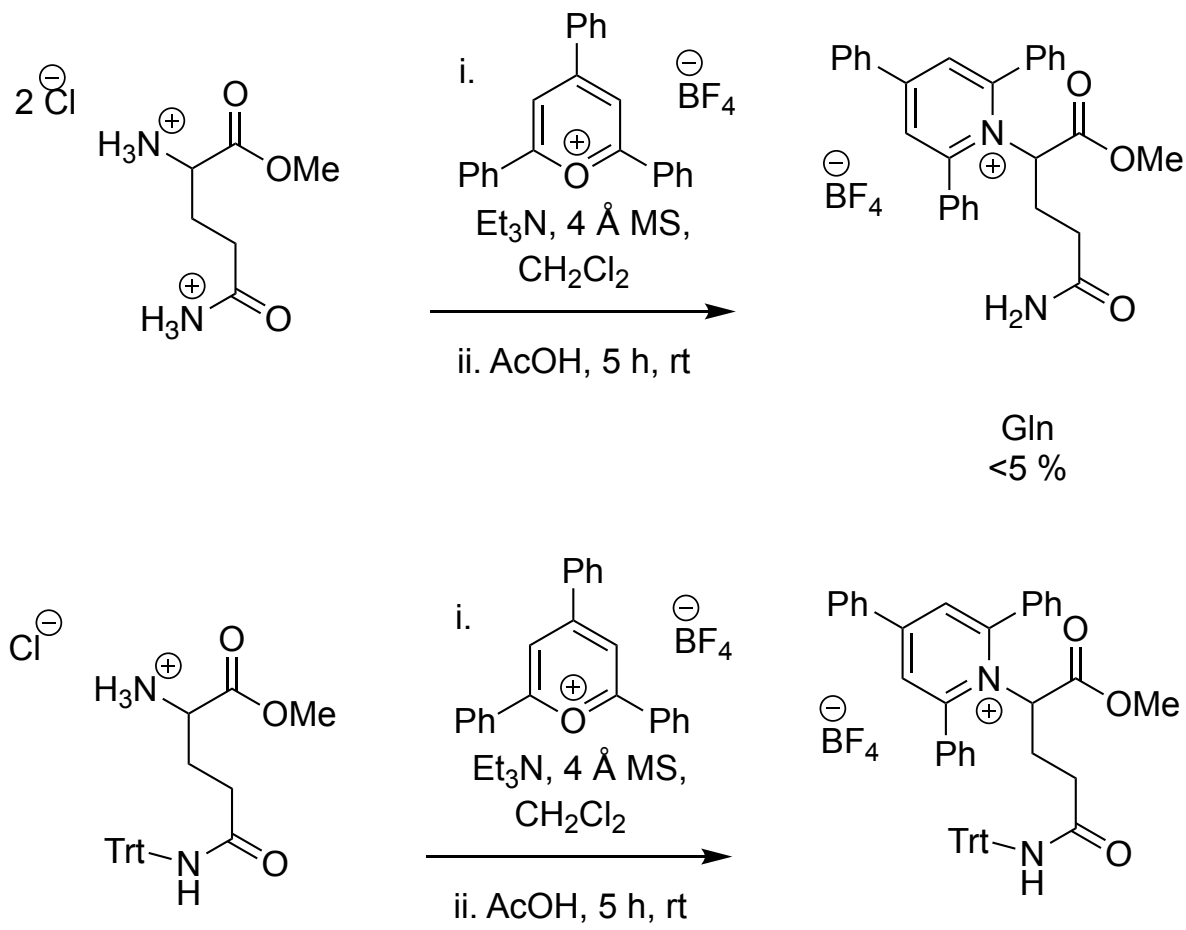<smiles></smiles>

$$
\begin{aligned}
& \text { Gln } \\
& <5 \%
\end{aligned}
$$

Unprotected and protected glutamine formed pyridinium salt in a yield of less than $5 \%$ and was not used in the cross-coupling scope.

\section{General Procedure B: Conversion of Amino Esters to Pyridinium Salts}<smiles>[R]C([NH3+])C(=O)OC</smiles>

Amino acid methyl ester (1.0 equiv), 2,4,6-triphenylpyrylium tetrafluoroborate (1.0 equiv), and crushed $4 \AA \mathrm{MS}(0.5 \mathrm{~g}$ per a mmol) were added to a round-bottomed flask. The flask was fitted with a septum and parafilmed. A vent needle was added, and $\mathrm{CH}_{2} \mathrm{Cl}_{2}(0.5 \mathrm{M})$ and then $\mathrm{Et}_{3} \mathrm{~N}$ (2.0 equiv) were added. The vent needle was removed, and the mixture was stirred for $20 \mathrm{~min}$ at room temperature. The vent needle was replaced, and $\mathrm{AcOH}$ (2.0 equiv) was added. The vent needle was removed, and the mixture was stirred for $5 \mathrm{~h}$ at room temperature. The mixture was filtered through a short pad of celite. The flask and celite were then rinsed with $\mathrm{CH}_{2} \mathrm{Cl}_{2}(25 \mathrm{~mL})$. The filtrate was then washed with $\mathrm{HCl}(1 \mathrm{M}, 4 \times 60 \mathrm{~mL})$, sat. $\mathrm{NaHCO}_{3}(4 \times 60 \mathrm{~mL})$, and sat. $\mathrm{NaCl}(1$ 
$\times 60 \mathrm{~mL})$. The organic layer was dried $\left(\mathrm{MgSO}_{4}\right)$, filtered, and concentrated. The resulting solid was dried under high vacuum. If diketone byproduct was present in more than $10 \%$, the product was purified by silica gel chromatography.<smiles></smiles>

1-(2-Methoxy-2-oxoethyl)-2,4,6-triphenylpyridin-1-ium tetrafluoroborate (3c). Prepared via General Procedure B on a $10 \mathrm{mmol}$ scale with glycine methyl ester. The crude material was purified via silica gel chromatography $\left(5 \rightarrow 15 \%\right.$ acetone $\left./ \mathrm{CH}_{2} \mathrm{Cl}_{2}\right)$ to give pyridinium salt $\mathbf{3 c}$ (4.24 g, 91\%) as a dark red solid (mp 164-166 $\left.{ }^{\circ} \mathrm{C}\right):{ }^{1} \mathrm{H}$ NMR (400 MHz, $\left.\mathrm{CDCl}_{3}\right) \delta 7.96(\mathrm{~s}, 2 \mathrm{H}), 7.84-$ $7.78(\mathrm{~m}, 3 \mathrm{H}), 7.74-7.51(\mathrm{~m}, 12 \mathrm{H}), 5.11(\mathrm{~s}, 2 \mathrm{H}), 3.54(\mathrm{~s}, 3 \mathrm{H}) ;{ }^{13} \mathrm{C} \mathrm{NMR}\left(101 \mathrm{MHz}, \mathrm{CDCl}_{3}\right) \delta$ 167.5, 157.3, 157.0, 133.9, 132.6, 132.1, 131.5, 129.9, 129.5, 129.0, 128.3, 126.3, 56.4, 53.4; ${ }^{19} \mathrm{~F}$ $\operatorname{NMR}\left(565 \mathrm{MHz}, \mathrm{CDCl}_{3}\right) \delta-153.40$ (minor, ${ }^{11} \mathrm{BF}_{4}$ ), -153.45 (major, ${ }^{10} \mathrm{BF}_{4}$ ); FTIR (neat) 3064, 1751, 1624, 1234, 1058, $702 \mathrm{~cm}^{-1}$; HRMS (ESI+) $\left[\mathrm{M}-\mathrm{BF}_{4}\right]^{+}$calculated for $\mathrm{C}_{26} \mathrm{H}_{22} \mathrm{NO}_{2}: 380.1645$, found 380.1637 .<smiles>COC(=O)C(c1ccccc1)n1c(-c2ccccc2)cc(-c2ccccc2)cc1=O</smiles>

\section{1-(1-Methoxy-1-oxopropan-2-yl)-2,4,6-triphenylpyridin-1-ium tetrafluoroborate (3a).}

Prepared via General Procedure B on a $10 \mathrm{mmol}$ scale with alanine methyl ester to give 3a (4.42 g, 92\%) as a light yellow solid: ${ }^{1} \mathrm{H}$ NMR $\left(400 \mathrm{MHz}, \mathrm{CDCl}_{3}\right) \delta 7.93(\mathrm{~s}, 2 \mathrm{H}), 7.85-7.48(\mathrm{~m}, 15 \mathrm{H})$, $5.56(\mathrm{q}, J=7.2 \mathrm{~Hz}, 1 \mathrm{H}), 3.67$ (s, 3H), $1.50(\mathrm{~d}, J=7.2 \mathrm{~Hz}, 3 \mathrm{H}) ;{ }^{13} \mathrm{C} \mathrm{NMR}\left(101 \mathrm{MHz}, \mathrm{CDCl}_{3}\right) \delta$ 169.0, 157.0, 156.8, 134.0, 132.7, 132.3, 131.5, 129.7, 129.2, 128.5, 127.9, 64.6, 53.8, 17.3 (1 carbon missing due to signal broadening as stated in the prior synthesis $\left.{ }^{10}\right) ;{ }^{19} \mathrm{~F}$ NMR $(376 \mathrm{MHz}$, $\left.\mathrm{CDCl}_{3}\right) \delta-153.18$ (minor, ${ }^{11} \mathrm{BF}_{4}$ ), -153.23 (major, ${ }^{10} \mathrm{BF}_{4}$ ). The spectral data matches that previously reported in the literature. ${ }^{10}$<smiles></smiles>

\section{1-(1-(Methoxy(methyl)amino)-1-oxopropan-2-yl)-2,4,6-triphenylpyridin-1-ium}

tetrafluoroborate (3d). Prepared via General Procedure B on a $5 \mathrm{mmol}$ scale with a Weinreb 
amide derived from alanine to give pyridinium salt 3d (1.26 g, 49\%) as a pale yellow solid (mp 125-127 $\left.{ }^{\circ} \mathrm{C}\right):{ }^{1} \mathrm{H}$ NMR $\left(400 \mathrm{MHz}, \mathrm{CDCl}_{3}\right) \delta 7.99$ (s, 2H), 7.87 (s, 2H), $7.81-7.79$ (m, 2H), 7.61$7.51(\mathrm{~m}, 11 \mathrm{H}), 5.84(\mathrm{q}, J=7.2 \mathrm{~Hz}, 1 \mathrm{H}), 3.09(\mathrm{~s}, 3 \mathrm{H}), 3.02(\mathrm{~s}, 3 \mathrm{H}), 1.34(\mathrm{~d}, J=7.2 \mathrm{~Hz}, 3 \mathrm{H}) ;{ }^{13} \mathrm{C}$ $\operatorname{NMR}\left(101 \mathrm{MHz}, \mathrm{CDCl}_{3}\right) \delta 157.7,155.9,134.4,134.1,132.2,131.0,129.8,129.6,129.2,128.9$, 128.4, 127.9, 67.9, 60.8, 32.9, 17.9; ${ }^{19} \mathrm{~F}$ NMR $\left(376 \mathrm{MHz}, \mathrm{CDCl}_{3}\right) \delta-153.47$ (minor, ${ }^{11} \mathrm{BF}_{4}$ ), 153.52 (major, ${ }^{10} \mathrm{BF}_{4}$ ); FTIR (neat) 2924, 1674, 1620, 1056, $704 \mathrm{~cm}^{-1}$; HRMS (ESI+) [M-BF $]^{+}$ calculated for $\mathrm{C}_{28} \mathrm{H}_{27} \mathrm{~N}_{2} \mathrm{O}_{2}: 423.2067$, found 423.2067 .

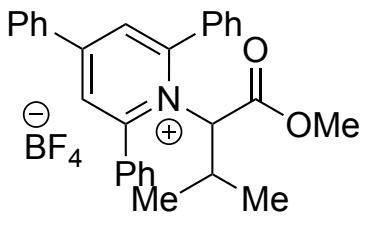

1-(1-Methoxy-3-methyl-1-oxobutan-2-yl)-2,4,6-triphenylpyridin-1-ium tetrafluoroborate (3e). Prepared via General Procedure B on a $5 \mathrm{mmol}$ scale with valine methyl ester. The crude material was purified via silica gel chromatography $\left(5 \rightarrow 15 \%\right.$ acetone $\left./ \mathrm{CH}_{2} \mathrm{Cl}_{2}\right)$ to give pyridinium salt 3e (1.47 g, 62\%) as a yellow/green solid (mp 115-121 $\left.{ }^{\circ} \mathrm{C}\right):{ }^{1} \mathrm{H} \mathrm{NMR}\left(400 \mathrm{MHz}, \mathrm{CDCl}_{3}\right) \delta 8.03$ (s, 2H), $7.94-7.88(\mathrm{~m}, 2 \mathrm{H}), 7.75-7.68(\mathrm{~m}, 2 \mathrm{H}), 7.66-7.51(\mathrm{~m}, 11 \mathrm{H}), 5.16(\mathrm{~d}, J=10.2 \mathrm{~Hz}, 1 \mathrm{H})$, $3.77(\mathrm{~s}, 3 \mathrm{H}), 2.10(\mathrm{dp}, J=10.2,6.5 \mathrm{~Hz}, 1 \mathrm{H}), 0.76(\mathrm{~d}, J=8 \mathrm{~Hz}, 6 \mathrm{H}) ;{ }^{13} \mathrm{C}$ NMR $\left(101 \mathrm{MHz}, \mathrm{CDCl}_{3}\right)$ $\delta 167.1,157.2,133.3,133.0,132.1,131.9,130.0,129.7,129.5,129.1,128.8,128.0,73.7,53.9$, 30.1, 22.5, 19.4; ${ }^{19} \mathrm{~F}$ NMR (376 MHz, $\left.\mathrm{CDCl}_{3}\right) \delta-153.20$ (minor, ${ }^{11} \mathrm{BF}_{4}$ ), -153.25 (major, ${ }^{10} \mathrm{BF}_{4}$ ); FTIR (neat) 3066, 1754, 1619, 1283, 1057, $705 \mathrm{~cm}^{-1}$; HRMS (ESI+) [M-BF $]^{+}$calculated for $\mathrm{C}_{29} \mathrm{H}_{28} \mathrm{NO}_{2}: 422.2115$, found 422.2109 .

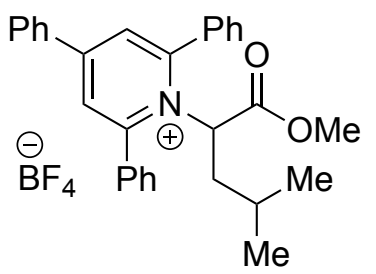

1-(1-Methoxy-4-methyl-1-oxopentan-2-yl)-2,4,6-triphenylpyridin-1-ium tetrafluoroborate (3f). Prepared via General Procedure B on a $10 \mathrm{mmol}$ scale with leucine methyl ester. The crude material was purified via silica chromatography $\left(5 \rightarrow 15 \%\right.$ acetone/ $\left.\mathrm{CH}_{2} \mathrm{Cl}_{2}\right)$ to give pyridinium salt 3f $(2.32 \mathrm{~g}, 44 \%)$ as a light yellow solid: ${ }^{1} \mathrm{H}$ NMR $\left(600 \mathrm{MHz}, \mathrm{CDCl}_{3}\right) \delta 7.91(\mathrm{~s}, 2 \mathrm{H}), 7.85-7.79$ (m, 2H), $7.78-7.69(\mathrm{~m}, 2 \mathrm{H}), 7.62-7.46(\mathrm{~m}, 11 \mathrm{H}), 5.47$ (dd, $J=8.6,3.6 \mathrm{~Hz}, 1 \mathrm{H}), 3.74(\mathrm{~s}, 3 \mathrm{H})$, 1.77 (ddd, $J=14.1,8.4,3.6 \mathrm{~Hz}, 1 \mathrm{H}), 1.56$ (ddd, $J=14.3,8.6,5.8 \mathrm{~Hz}, 1 \mathrm{H}), 1.33$ (ddd, $J=12.7$, 
8.2, $6.3 \mathrm{~Hz}, 1 \mathrm{H}), 0.58(\mathrm{~d}, J=6.6 \mathrm{~Hz}, 3 \mathrm{H}), 0.43(\mathrm{~d}, J=6.6 \mathrm{~Hz}, 3 \mathrm{H}) ;{ }^{13} \mathrm{C} \mathrm{NMR}\left(101 \mathrm{MHz}, \mathrm{CDCl}_{3}\right)$ $\delta 169.1,156.9,156.8,133.9,132.7,132.4,131.6,129.7,129.5,129.2,128.6,128.1,67.5,53.9$, 40.5, 26.3, 22.5, 20.7; ${ }^{19} \mathrm{~F}$ NMR (376 MHz, $\mathrm{CDCl}_{3}$ ) $\delta-153.04$ (minor, ${ }^{11} \mathrm{BF}_{4}$ ), -153.09 (major, ${ }^{10} \mathrm{BF}_{4}$ ). The spectral data matches that previously reported in the literature. ${ }^{10}$<smiles></smiles>

\section{1-(1-Methoxy-1-oxo-3-phenylpropan-2-yl)-2,4,6-triphenylpyridin-1-ium tetrafluoroborate}

(3g). Prepared via General Procedure B on a $10 \mathrm{mmol}$ scale with phenylalanine methyl ester. The crude material was purified via silica gel chromatography $\left(5 \rightarrow 15 \%\right.$ acetone $\left./ \mathrm{CH}_{2} \mathrm{Cl}_{2}\right)$ to give pyridinium salt $3 \mathrm{~g}$ (3.23 g, 58\%) as a yellow solid: ${ }^{1} \mathrm{H} \mathrm{NMR}\left(600 \mathrm{MHz}, \mathrm{CDCl}_{3}\right) \delta 7.93(\mathrm{~s}, 2 \mathrm{H})$, $7.86-7.78$ (m, 3H), $7.69-7.38$ (m, 12H), 7.09 (ddd, $J=14.2,7.8,6.1 \mathrm{~Hz}, 3 \mathrm{H}), 6.80-6.74(\mathrm{~m}$, 2H), $5.64(\mathrm{dd}, J=8.0,4.1 \mathrm{~Hz}, 1 \mathrm{H}), 3.69$ (s, 3H), 3.47 (dd, $J=14.5,4.1 \mathrm{~Hz}, 1 \mathrm{H}), 2.93$ (dd, $J=$ $14.5,8.1 \mathrm{~Hz}, 1 \mathrm{H}) ;{ }^{13} \mathrm{C}$ NMR $\left(101 \mathrm{MHz}, \mathrm{CDCl}_{3}\right) \delta 167.9,157.1,157.0,136.3,133.8,132.5,132.3$, 131.7, 129.8, 129.6, 129.2, 129.1, 128.7, 128.6, 127.9, 127.3, 70.3, 53.9, 37.8; ${ }^{19} \mathrm{~F}$ NMR (376 $\left.\mathrm{MHz}, \mathrm{CDCl}_{3}\right) \delta-152.85$ (minor, ${ }^{11} \mathrm{BF}_{4}$ ), -152.91 (major, ${ }^{10} \mathrm{BF}_{4}$ ). The spectral data matches that previously reported in the literature. ${ }^{10}$

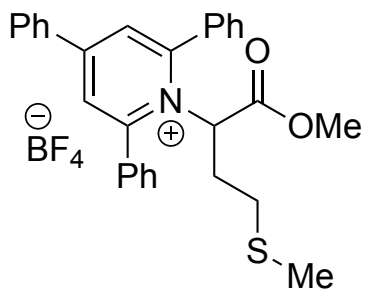

\section{1-(1-Methoxy-4-(methylthio)-1-oxobutan-2-yl)-2,4,6-triphenylpyridin-1-ium}

tetrafluoroborate (3h). Prepared via General Procedure B on a $5 \mathrm{mmol}$ scale with methionine methyl ester. The crude material was purified via silica gel chromatography $(5 \rightarrow 15 \%$ acetone $\left./ \mathrm{CH}_{2} \mathrm{Cl}_{2}\right)$ to give pyridinium salt $\mathbf{3 h}(1.86 \mathrm{~g}, 69 \%)$ as a light yellow solid: ${ }^{1} \mathrm{H}$ NMR (400 $\left.\mathrm{MHz}, \mathrm{CDCl}_{3}\right) \delta 7.93(\mathrm{~d}, J=3.7 \mathrm{~Hz}, 2 \mathrm{H}), 7.83(\mathrm{dt}, J=6.7,1.4 \mathrm{~Hz}, 3 \mathrm{H}), 7.70-7.47(\mathrm{~m}, 12 \mathrm{H}), 6.04$ $(\mathrm{dt}, J=9.4,2.5 \mathrm{~Hz}, 1 \mathrm{H}), 3.73(\mathrm{~s}, 3 \mathrm{H}), 2.43-2.20(\mathrm{~m}, 3 \mathrm{H}), 1.87(\mathrm{~m}, 4 \mathrm{H}) ;{ }^{13} \mathrm{C}$ NMR $(101 \mathrm{MHz}$, $\left.\mathrm{CDCl}_{3}\right) \delta 169.2,157.2,134.2,132.8,132.3,131.5,129.8,129.2,128.7,66.7,53.8,31.7,30.6,14.8$ ( 3 carbons missing due to signal broadening as stated in the literature $\left.{ }^{10}\right) ;{ }^{19} \mathrm{~F}$ NMR $(376 \mathrm{MHz}$, 
$\left.\mathrm{CDCl}_{3}\right) \delta-152.85$ (minor, ${ }^{11} \mathrm{BF}_{4}$ ), -152.91 (major, ${ }^{10} \mathrm{BF}_{4}$ ). The spectral data matches that previously reported in the literature. ${ }^{10}$

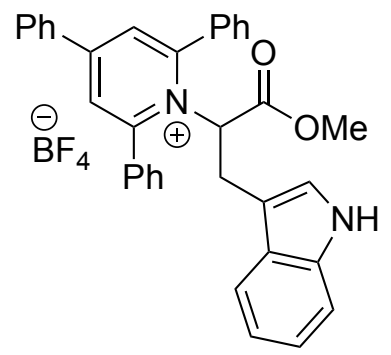

\section{1-(3-(1H-Indol-3-yl)-1-methoxy-1-oxopropan-2-yl)-2,4,6-triphenylpyridin-1-ium}

tetrafluoroborate (3i). Prepared via General Procedure B on a $10 \mathrm{mmol}$ scale with tryptophan methyl ester to give pyridinium salt $3 \mathbf{i}(1.46 \mathrm{~g}, 95 \%)$ as an orange solid (mp $\left.113-116{ }^{\circ} \mathrm{C}\right):{ }^{1} \mathrm{H} \mathrm{NMR}$ $\left(400 \mathrm{MHz}, \mathrm{CDCl}_{3}\right) \delta 9.51(\mathrm{~s}, 1 \mathrm{H}), 8.23-8.17(\mathrm{~m}, 1 \mathrm{H}), 7.92-7.80(\mathrm{~m}, 5 \mathrm{H}), 7.61-7.36(\mathrm{~m}, 12 \mathrm{H})$, 7.07 (ddd, $J=8.1,5.8,2.3 \mathrm{~Hz}, 1 \mathrm{H}), 6.86-6.73(\mathrm{~m}, 3 \mathrm{H}), 5.74$ (dd, $J=10.3,4.4 \mathrm{~Hz}, 1 \mathrm{H}), 3.79$ (s, $3 \mathrm{H}), 3.32(\mathrm{dd}, J=15.7,4.4 \mathrm{~Hz}, 1 \mathrm{H}), 3.14(\mathrm{dd}, J=15.7,10.4 \mathrm{~Hz}, 1 \mathrm{H}) ;{ }^{13} \mathrm{C} \mathrm{NMR}\left(101 \mathrm{MHz}, \mathrm{CDCl}_{3}\right)$ $\delta 168.4,157.5,157.0,136.2,133.2,132.9,131.9,131.5,129.9,129.2,128.8,127.3,126.4,124.0$, 122.0, 119.4, 117.3, 116.8, 112.4, 106.1, 69.6, 54.0, 27.0; ${ }^{19} \mathrm{~F}$ NMR $\left(376 \mathrm{MHz}, \mathrm{CDCl}_{3}\right) \delta-151.94$ (minor, ${ }^{11} \mathrm{BF}_{4}$ ), -152.00 (major, ${ }^{10} \mathrm{BF}_{4}$ ); FTIR (neat) 3392, 3059, 1753, 1618,1058, $702 \mathrm{~cm}^{-1}$; HRMS (ESI+) $\left[\mathrm{M}-\mathrm{BF}_{4}\right]^{+}$calculated for $\mathrm{C}_{35} \mathrm{H}_{29} \mathrm{~N}_{2} \mathrm{O}_{2}: 509.2224$, found 509.2224.

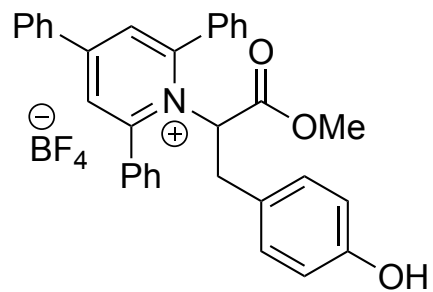

\section{1-(3-(4-Hydroxyphenyl)-1-methoxy-1-oxopropan-2-yl)-2,4,6-triphenylpyridin-1-ium}

tetrafluoroborate (3j). Prepared via General Procedure B on a $5 \mathrm{mmol}$ scale with tyrosine methyl ester. The crude material was purified via silica gel chromatography $\left(5 \rightarrow 15 \%\right.$ acetone $\left./ \mathrm{CH}_{2} \mathrm{Cl}_{2}\right)$ to give pyridinium salt $\mathbf{3 j}(1.40 \mathrm{~g}, 50 \%)$ as a yellow solid (mp $\left.143-147^{\circ} \mathrm{C}\right):{ }^{1} \mathrm{H}$ NMR (400 MHz, $\left.\mathrm{CDCl}_{3}\right) \delta 7.88(\mathrm{~s}, 2 \mathrm{H}), 7.88-7.77(\mathrm{~m}, 2 \mathrm{H}), 7.75-7.41(\mathrm{~m}, 13 \mathrm{H}), 6.80(\mathrm{~s}, 1 \mathrm{H}), 6.63-6.56(\mathrm{~m}$, 2H), $6.42-6.36(\mathrm{~m}, 2 \mathrm{H}), 5.57(\mathrm{t}, J=6.7 \mathrm{~Hz}, 1 \mathrm{H}), 3.69(\mathrm{~s}, 3 \mathrm{H}), 3.11(\mathrm{dd}, J=14.8,7.1 \mathrm{~Hz}, 1 \mathrm{H})$, $2.88(\mathrm{dd}, J=14.8,6.3 \mathrm{~Hz}, 1 \mathrm{H}) ;{ }^{13} \mathrm{C} \mathrm{NMR}\left(101 \mathrm{MHz}, \mathrm{CDCl}_{3}\right) \delta 167.9,156.9,156.3,133.3,132.9$, $132.1,131.9,130.0,129.8,129.7,129.3,128.9,128.6,127.7,125.9,116.2,70.8,53.9,36.6 ;{ }^{19} \mathrm{~F}$ NMR (376 MHz, $\left.\mathrm{CDCl}_{3}\right) \delta-151.84$ (minor, ${ }^{11} \mathrm{BF}_{4}$ ), -151.89 (major, ${ }^{10} \mathrm{BF}_{4}$ ); FTIR (neat) 3445, 
$3064,2956,1754,1618,1227,1059,703 \mathrm{~cm}^{-1}$; HRMS $(\mathrm{ESI}+)\left[\mathrm{M}-\mathrm{BF}_{4}\right]^{+}$calculated for $\mathrm{C}_{33} \mathrm{H}_{28} \mathrm{NO}_{3}: 486.2064$, found 486.2077 .

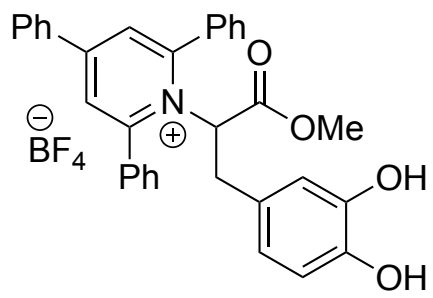

\section{1-(3-(3,4-Dihydroxyphenyl)-1-methoxy-1-oxopropan-2-yl)-2,4,6-triphenylpyridin-1-ium}

tetrafluoroborate (3k). Prepared via General Procedure B on a $10 \mathrm{mmol}$ scale with levadopa methyl ester. The crude material was purified via silica gel chromatography $(5 \rightarrow 15 \%$ acetone $\left./ \mathrm{CH}_{2} \mathrm{Cl}_{2}\right)$ to give pyridinium salt $3 \mathbf{k}(1.48 \mathrm{~g}, 28 \%)$ as a dark yellow solid (mp 138-140 $\left.{ }^{\circ} \mathrm{C}\right)$ : ${ }^{1} \mathrm{H}$ NMR (400 MHz, $\left.\mathrm{CDCl}_{3}\right) \delta 7.93-7.80(\mathrm{~m}, 5 \mathrm{H}), 7.60-7.43(\mathrm{~m}, 11 \mathrm{H}), 7.13(\mathrm{~s}, 1 \mathrm{H}), 6.65$ (d, $J$ $=8.1 \mathrm{~Hz}, 1 \mathrm{H}), 6.38(\mathrm{~d}, J=2.1 \mathrm{~Hz}, 1 \mathrm{H}), 5.98(\mathrm{dd}, J=8.2,2.2 \mathrm{~Hz}, 2 \mathrm{H}), 5.58(\mathrm{dd}, J=8.7,5.7 \mathrm{~Hz}$, 1H), 3.74 (s, 3H), $3.01-2.93$ (m, 2H); ${ }^{13} \mathrm{C}$ NMR (101 MHz, $\left.\mathrm{CDCl}_{3}\right) \delta$ 168.1, 157.2, 144.5, 144.4, 133.2, 133.0, 132.0, 131.9, 130.0, 129.4, 128.8, 125.8, 120.8, 115.6, 115.1, 71.0, 54.1, 36.1 (3 carbons missing due to signal broadening); ${ }^{19} \mathrm{~F} \mathrm{NMR}\left(376 \mathrm{MHz}, \mathrm{CDCl}_{3}\right) \delta-150.70$ (minor, ${ }^{11} \mathrm{BF}_{4}$ ), -150.75 (major, ${ }^{10} \mathrm{BF}_{4}$ ); FTIR (neat) 3433, 2924, 1752, 1619, 1270, 1061, 765, $703 \mathrm{~cm}^{-1}$; HRMS $(\mathrm{ESI}+)\left[\mathrm{M}-\mathrm{BF}_{4}\right]^{+}$calculated for $\mathrm{C}_{33} \mathrm{H}_{28} \mathrm{NO}_{4}:$ 502.2013, found 502.2016.

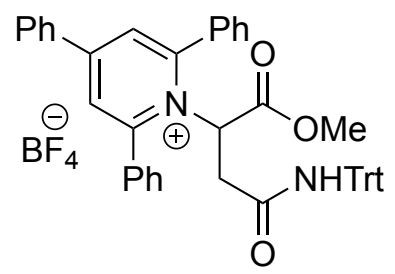

\section{1-(1,5-Dimethoxy-1,5-dioxopentan-2-yl)-2,4,6-triphenylpyridin-1-ium}

tetrafluoroborate

(3l). Prepared via General Procedure B on a $5 \mathrm{mmol}$ scale with trityl protected asparagine methyl ester. The crude material was purified via silica gel chromatography $\left(5 \rightarrow 15 \%\right.$ acetone $\left./ \mathrm{CH}_{2} \mathrm{Cl}_{2}\right)$ to give pyridinium salt $3 \mathrm{l}(0.878 \mathrm{~g}, 23 \%)$ as a light orange solid $\left(\mathrm{mp} 165-166{ }^{\circ} \mathrm{C}\right):{ }^{1} \mathrm{H}$ NMR $(400$ $\left.\mathrm{MHz}, \mathrm{CDCl}_{3}\right) \delta 8.28-7.80(\mathrm{~m}, 7 \mathrm{H}), 7.73-7.45(\mathrm{~m}, 11 \mathrm{H}), 7.43-7.34(\mathrm{~m}, 1 \mathrm{H}), 7.32-7.27(\mathrm{~m}$, 4H), $7.25-7.20$ (m, 2H), $7.17-7.07$ (m, 7H), 6.22 (dd, $J=9.3,2.0 \mathrm{~Hz}, 1 \mathrm{H}), 3.58$ (dd, $J=17.3$, $2.1 \mathrm{~Hz}, 1 \mathrm{H}), 3.53(\mathrm{~s}, 3 \mathrm{H}), 2.97(\mathrm{dd}, J=17.2,9.3 \mathrm{~Hz}, 1 \mathrm{H}) ;{ }^{13} \mathrm{C} \mathrm{NMR}\left(101 \mathrm{MHz}, \mathrm{CDCl}_{3}\right) \delta 167.6$, 166.4, 156.7, 144.5, 133.6, 132.7, 131.7, 129.9, 129.3, 128.8, 128.4, 128.2, 127.9, 127.2, 126.9, 126.5, 70.7, 64.1, 53.6, 38.3 ( 1 carbon missing due to signal broadening); ${ }^{19} \mathrm{~F}$ NMR (376 MHz, 
$\left.\mathrm{CDCl}_{3}\right) \delta-151.89$ (minor, ${ }^{11} \mathrm{BF}_{4}$ ), -151.95 (major, ${ }^{10} \mathrm{BF}_{4}$ ); FTIR (neat) 3374, 3061, 2954, 1754, $1620,1493,1059,765,701 \mathrm{~cm}^{-1}$; HRMS (ESI+) $\left[\mathrm{M}-\mathrm{BF}_{4}\right]^{+}$calculated for $\mathrm{C}_{47} \mathrm{H}_{39} \mathrm{~N}_{2} \mathrm{O}_{3}: 679.2955$, found 679.2977 .

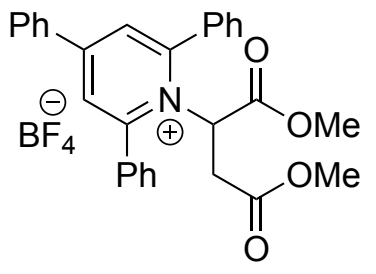

\section{1-(1,4-Dimethoxy-1,4-dioxobutan-2-yl)-2,4,6-triphenylpyridin-1-ium tetrafluoroborate}

(3m). Prepared via General Procedure B on a $10 \mathrm{mmol}$ scale with aspartic acid methyl ester. The crude material was purified via silica gel chromatography $\left(5 \rightarrow 15 \%\right.$ acetone $\left./ \mathrm{CH}_{2} \mathrm{Cl}_{2}\right)$ to give pyridinium salt $3 \mathrm{~m}(1.21 \mathrm{~g}, 22 \%)$ as a orange solid (mp 90-92 $\left.{ }^{\circ} \mathrm{C}\right):{ }^{1} \mathrm{H}$ NMR $\left(400 \mathrm{MHz}, \mathrm{CDCl}_{3}\right) \delta$ $7.97(\mathrm{~s}, 3 \mathrm{H}), 7.87-7.73(\mathrm{~m}, 3 \mathrm{H}), 7.72-7.42(\mathrm{~m}, 11 \mathrm{H}), 6.26(\mathrm{dd}, J=9.8,1.8 \mathrm{~Hz}, 1 \mathrm{H}), 3.63(\mathrm{~s}$, $3 \mathrm{H}), 3.54(\mathrm{~s}, 3 \mathrm{H}), 3.44(\mathrm{dd}, J=17.5,1.8 \mathrm{~Hz}, 1 \mathrm{H}), 2.53(\mathrm{dd}, J=17.5,9.8 \mathrm{~Hz}, 1 \mathrm{H}) ;{ }^{13} \mathrm{C} \mathrm{NMR}(101$ $\left.\mathrm{MHz}_{\mathrm{CDCl}}\right) \delta 169.8,168.0,157.6,134.1,132.5,131.7,129.8,129.4,128.7,64.1,54.0,52.6$, 36.0 (4 carbons missing due to signal broadening); ${ }^{19} \mathrm{~F} \mathrm{NMR}\left(376 \mathrm{MHz}, \mathrm{CDCl}_{3}\right) \delta-152.83$ (minor, ${ }^{11} \mathrm{BF}_{4}$ ), -152.89 (major, ${ }^{10} \mathrm{BF}_{4}$ ); FTIR (neat) 3065, 2956, 1741, 1622, 1058, 765, $704 \mathrm{~cm}^{-1}$; HRMS (ESI+) $\left[\mathrm{M}-\mathrm{BF}_{4}\right]^{+}$calculated for $\mathrm{C}_{29} \mathrm{H}_{26} \mathrm{NO}_{4}: 452.1856$, found 452.1872 .

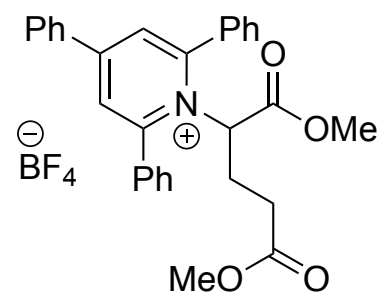

\section{1-(1,5-Dimethoxy-1,5-dioxopentan-2-yl)-2,4,6-triphenylpyridin-1-ium tetrafluoroborate}

(3n). Prepared via General Procedure B on a $10 \mathrm{mmol}$ scale with glutamic acid methyl ester. The crude material was purified via silica gel chromatography $\left(5 \rightarrow 15 \%\right.$ acetone $\left./ \mathrm{CH}_{2} \mathrm{Cl}_{2}\right)$ to give pyridinium salt 3n (2.32 g, 42\%) as a light yellow solid (mp 169-172 $\left.{ }^{\circ} \mathrm{C}\right)$ : ${ }^{1} \mathrm{H}$ NMR (600 MHz, $\left.\mathrm{CDCl}_{3}\right) \delta 7.93(\mathrm{~s}, 2 \mathrm{H}), 7.85-7.80(\mathrm{~m}, 2 \mathrm{H}), 7.75(\mathrm{~s}, 2 \mathrm{H}), 7.63-7.46(\mathrm{~m}, 11 \mathrm{H}), 5.62(\mathrm{dd}, J=7.1$, $5.3 \mathrm{~Hz}, 1 \mathrm{H}), 3.71(\mathrm{~s}, 3 \mathrm{H}), 3.47(\mathrm{~s}, 3 \mathrm{H}), 2.30-2.03(\mathrm{~m}, 4 \mathrm{H}) ;{ }^{13} \mathrm{C} \mathrm{NMR}\left(101 \mathrm{MHz}, \mathrm{CDCl}_{3}\right) \delta 172.1$, 168.2, 157.2, 133.8, 132.5, 132.4, 131.7, 129.8, 129.3, 129.0, 128.6, 67.6, 54.0, 51.8, 30.7, 27.0 (2 carbons missing due to signal broadening); ${ }^{19} \mathrm{~F}$ NMR (565 MHz, $\left.\mathrm{CDCl}_{3}\right) \delta-153.10$ (minor, ${ }^{11} \mathrm{BF}_{4}$ ), 
-153.15 (major, ${ }^{10} \mathrm{BF}_{4}$ ); FTIR (neat) 2955, 1737, 1620, 1055, 768, $705 \mathrm{~cm}^{-1}$; HRMS (ESI+) [M$\left.\mathrm{BF}_{4}\right]^{+}$calculated for $\mathrm{C}_{30} \mathrm{H}_{28} \mathrm{NO}_{4}: 466.2013$, found 466.2010.

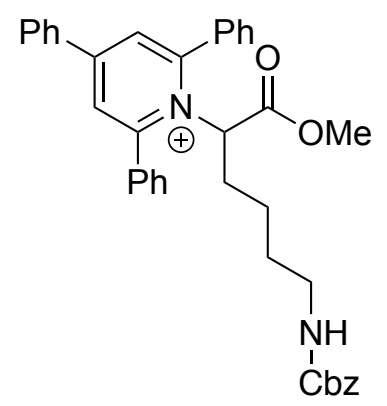

\section{1-(6-(((Benzyloxy)carbonyl)amino)-1-methoxy-1-oxohexan-2-yl)-2,4,6-triphenylpyridin-1-}

ium tetrafluoroborate (3o). Prepared via General Procedure B on a $10 \mathrm{mmol} \mathrm{scale}$ with lysine methyl ester. The crude material was purified via silica gel chromatography $(5 \rightarrow 15 \%$ acetone $\left./ \mathrm{CH}_{2} \mathrm{Cl}_{2}\right)$ to give pyridinium salt $30\left(5.25 \mathrm{~g}, 79 \%\right.$ ) as a light yellow solid (mp 94-97 ${ }^{\circ} \mathrm{C}$ ): ${ }^{1} \mathrm{H}$ NMR (400 MHz, $\left.\mathrm{CDCl}_{3}\right) \delta 7.94$ (s, 2H), 7.83 (d, $\left.J=6.6 \mathrm{~Hz}, 3 \mathrm{H}\right), 7.62-7.50$ (m, 13H), $7.38-$ $7.28(\mathrm{~m}, 5 \mathrm{H}), 5.51(\mathrm{t}, J=5.9 \mathrm{~Hz}, 1 \mathrm{H}), 5.32(\mathrm{dd}, J=7.8,5.3 \mathrm{~Hz}, 1 \mathrm{H}), 5.06(\mathrm{~s}, 2 \mathrm{H}), 3.70(\mathrm{~s}, 3 \mathrm{H})$, $3.05(\mathrm{q}, J=6.3 \mathrm{~Hz}, 2 \mathrm{H}), 1.96-1.00(\mathrm{~m}, 6 \mathrm{H}) ;{ }^{13} \mathrm{C} \mathrm{NMR}\left(101 \mathrm{MHz}, \mathrm{CDCl}_{3}\right) \delta$ 169.0, 157.1, 156.8, 137.1, 134.0, 132.5, 131.6, 129.9, 129.8, 129.4, 129.3, 128.6, 128.5, 128.4, 128.3, 128.1, 127.9, 69.2, 66.2, 53.8, 39.9, 31.1, 28.6, 24.3; ${ }^{19} \mathrm{~F}$ NMR (376 MHz, $\left.\mathrm{CDCl}_{3}\right) \delta-152.61$ (minor, ${ }^{11} \mathrm{BF}_{4}$ ), 152.66 (major, ${ }^{10} \mathrm{BF}_{4}$ ); FTIR (neat) 2952, 1751, 1620, 1247, 1056, $704 \mathrm{~cm}^{-1}$; HRMS (ESI+) [M$\left.\mathrm{BF}_{4}\right]^{+}$calculated for $\mathrm{C}_{38} \mathrm{H}_{37} \mathrm{~N}_{2} \mathrm{O}_{4}: 585.2748$, found 585.2741.

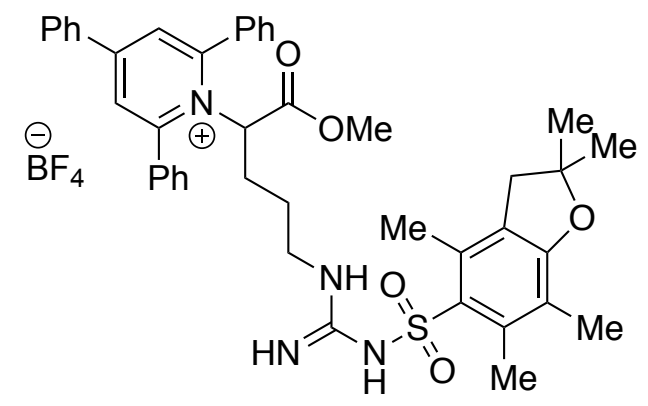

\section{1-(1-Methoxy-1-oxo-5-(3-((2,2,4,6,7-pentamethyl-2,3-dihydrobenzofuran-5-}

yl)sulfonyl)guanidino)pentan-2-yl)-2,4,6-triphenylpyridin-1-ium tetrafluoroborate (3p).

Prepared via General Procedure B on a $7 \mathrm{mmol}$ scale with arginine methyl ester. The crude material was purified via silica gel chromatography $\left(5 \rightarrow 15 \%\right.$ acetone $\left./ \mathrm{CH}_{2} \mathrm{Cl}_{2}\right)$ to give pyridinium salt $\mathbf{3 p}$ $(0.803 \mathrm{~g}, 17 \%)$ as an orange solid (mp $\left.103-106{ }^{\circ} \mathrm{C}\right):{ }^{1} \mathrm{H} \mathrm{NMR}\left(400 \mathrm{MHz}, \mathrm{CDCl}_{3}\right) \delta 8.04-7.48$ (m, 17H), $6.14(\mathrm{~s}, 1 \mathrm{H}), 5.82(\mathrm{~s}, 1 \mathrm{H}), 5.28(\mathrm{dd}, J=7.9,4.6 \mathrm{~Hz}, 1 \mathrm{H}), 3.68(\mathrm{~s}, 3 \mathrm{H}), 2.98(\mathrm{~m}, 4 \mathrm{H})$, 
$2.52(\mathrm{~s}, 3 \mathrm{H}), 2.47(\mathrm{~s}, 3 \mathrm{H}), 2.05(\mathrm{~s}, 3 \mathrm{H}), 1.94(\mathrm{ddt}, J=19.7,10.3,5.0 \mathrm{~Hz}, 1 \mathrm{H}), 1.68$ (qd, $J=9.0$, 7.3, $3.4 \mathrm{~Hz}, 1 \mathrm{H}), 1.44(\mathrm{~s}, 6 \mathrm{H}), 1.32(\mathrm{dt}, J=16.2,6.2 \mathrm{~Hz}, 1 \mathrm{H}), 1.06(\mathrm{~s}, 1 \mathrm{H}) ;{ }^{13} \mathrm{C} \mathrm{NMR}(101 \mathrm{MHz}$, $\left.\mathrm{CDCl}_{3}\right) \delta 168.3,158.5,156.9,156.1,138.3,133.4,132.8,132.2,132.2,132.0,129.9,129.8,129.6$, $128.9,128.8,128.7,128.5,128.4,124.5,117.4,86.3,68.7,54.0,43.3,39.1,29.3,29.3,28.7,27.3$, 19.4, 18.1, 12.6; ${ }^{19} \mathrm{~F}$ NMR (376 $\left.\mathrm{MHz}, \mathrm{CDCl}_{3}\right) \delta-150.53$ (minor, ${ }^{11} \mathrm{BF}_{4}$ ), -150.59 (major, ${ }^{10} \mathrm{BF}_{4}$ ); FTIR (neat) 3458, 3361, 2971, 1751, 1620, 1558, 1261, 1090, 913, $732 \mathrm{~cm}^{-1}$; HRMS (ESI+) [M$\left.\mathrm{BF}_{4}\right]^{+}$calculated for $\mathrm{C}_{43} \mathrm{H}_{47} \mathrm{~N}_{4} \mathrm{O}_{5} \mathrm{~S}: 731.3262$, found 731.3262 .<smiles></smiles>

1-(1-Cyclopropyl-2-methoxy-2-oxoethyl)-2,4,6-triphenylpyridin-1-ium tetrafluoroborate (3q). Prepared via General Procedure B on a $5 \mathrm{mmol}$ scale with cyclopropylglycine methyl ester. The crude material was purified via silica gel chromatography $\left(5 \rightarrow 15 \%\right.$ acetone $\left./ \mathrm{CH}_{2} \mathrm{Cl}_{2}\right)$ to give pyridinium salt 3q $(1.23 \mathrm{~g}, 50 \%)$ as a light yellow solid (mp 105-107 $\left.{ }^{\circ} \mathrm{C}\right):{ }^{1} \mathrm{H}$ NMR $(400 \mathrm{MHz}$, $\left.\mathrm{CDCl}_{3}\right) \delta 7.99(\mathrm{~s}, 2 \mathrm{H}), 7.91-7.87(\mathrm{~m}, 2 \mathrm{H}), 7.71-7.51(\mathrm{~m}, 13 \mathrm{H}), 4.70(\mathrm{dd}, J=8.8 \mathrm{~Hz}, 1 \mathrm{H}), 3.79$ $(\mathrm{s}, 3 \mathrm{H}), 0.68-0.49(\mathrm{~m}, 4 \mathrm{H}) 0.01(\mathrm{~m}, 1 \mathrm{H}) ;{ }^{13} \mathrm{C} \mathrm{NMR}\left(101 \mathrm{MHz}, \mathrm{CDCl}_{3}\right) \delta 168.9,157.3,133.9$, 133.0, 132.5, 131.6, 129.8, 129.4, 128.8, 74.8, 54.1, 13.3, 11.0, 5.4 (3 carbons missing due to signal broadening); ${ }^{19} \mathrm{~F} \mathrm{NMR}\left(376 \mathrm{MHz}, \mathrm{CDCl}_{3}\right) \delta-153.13$ (minor, $\left.{ }^{11} \mathrm{BF}_{4}\right),-153.18$ (major, ${ }^{10} \mathrm{BF}_{4}$ ); FTIR (neat) 3066, 2923, 1751, 1619,1056, 768, $705 \mathrm{~cm}^{-1}$; HRMS (ESI+) $\left[\mathrm{M}-\mathrm{BF}_{4}\right]^{+}$calculated for $\mathrm{C}_{29} \mathrm{H}_{26} \mathrm{NO}_{2}: 420.1958$, found 420.1956 .

\section{Mechanistic Experiments}

Test for Stereospecificity

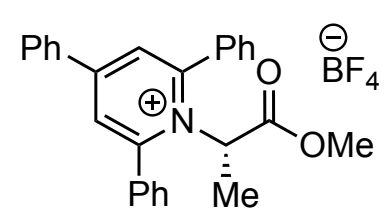

$(S)-3 a$ from L-alanine

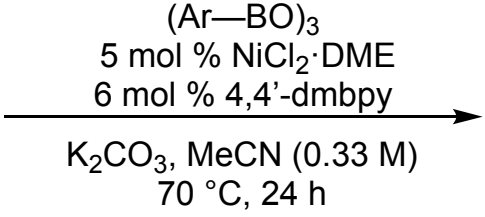

$70^{\circ} \mathrm{C}, 24 \mathrm{~h}$

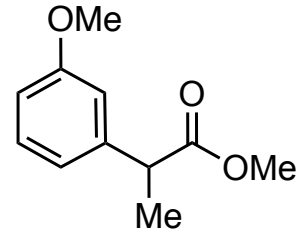

8, $>99 \%(\mathrm{NMR})$ racemic

In a $\mathrm{N}_{2}$-filled glovebox: To an oven-dried 1-dram vial was added $\mathrm{NiCl}_{2} \cdot \mathrm{DME}(1.1 \mathrm{mg}, 0.0050$ 
mmol, 5 mol \%), 4,4'-dimethyl-2,2'-dipyridyl (4,4'-dmbpy, $1.1 \mathrm{mg}, 0.0060 \mathrm{mmol}, 6 \mathrm{~mol} \%$ ), $\mathrm{K}_{2} \mathrm{CO}_{3}$ (38.7 mg, $0.28 \mathrm{mmol}, 2.8$ equiv), 3-(methoxy)phenylboroxine (32 mg, $0.080 \mathrm{mmol}, 0.80$ equiv), and $(S)-3 a(48 \mathrm{mg}, 0.10 \mathrm{mmol}, 1.0$ equiv). Anhydrous acetonitrile $(0.3 \mathrm{~mL}, 0.33 \mathrm{M})$ was added to the vial. The vial was then equipped with a micro stir bar, capped with a Teflon-coated cap, and removed from the glovebox. The resulting reaction mixture was stirred vigorously at 70 ${ }^{\circ} \mathrm{C}$ for $24 \mathrm{~h}$. The mixture was then diluted with $\mathrm{Et}_{2} \mathrm{O}$ (approx. $1.5 \mathrm{~mL}$ ) and filtered through a short plug of silica gel. The filter cake was washed with $\mathrm{Et}_{2} \mathrm{O}(5 \times 1 \mathrm{~mL})$, and the resulting solution was concentrated. The yield of product 8 was determined to be quantitative by ${ }^{1} \mathrm{H}-\mathrm{NMR}$ analysis with 1,3,5-trimethoxybenzene as an internal standard. A small sample of the product was purified via preparatory thin-layer chromatography (10\% ethyl acetate/hexanes), and the enantiomeric excess was determined to be $0 \%$ by chiral HPLC analysis (CHIRALPAK IC, $1.0 \mathrm{~mL} / \mathrm{min}, 1 \%$ IPA/Hexane, $\lambda=210 \mathrm{~nm})$; tR $($ enantiomer $\mathrm{A})=17.13 \mathrm{~min}$, tR $($ enantiomer $\mathrm{B})=19.05 \mathrm{~min}$.

\section{Radical Clock Experiment}

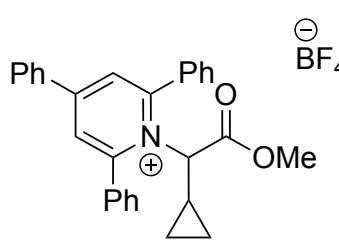

$3 q$

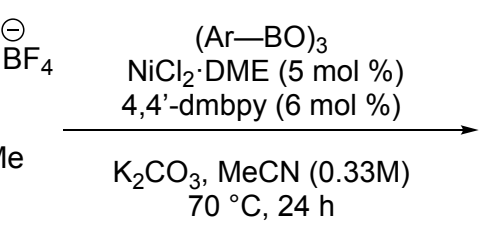

$70^{\circ} \mathrm{C}, 24 \mathrm{~h}$

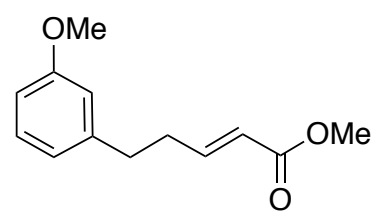

27, 56\% (NMR)

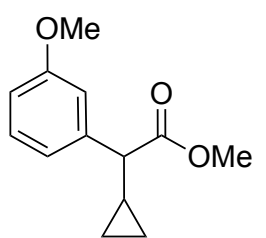

28, $0 \%$

In a $\mathrm{N}_{2}$-filled glovebox: To an oven-dried 1-dram vial was added $\mathrm{NiCl}_{2} \cdot \mathrm{DME}(1.1 \mathrm{mg}, 0.0050$ mmol, 5 mol \%), 4,4'-dimethyl-2,2'-dipyridyl (4,4'-dmbpy, $1.1 \mathrm{mg}, 0.0060 \mathrm{mmol}, 6 \mathrm{~mol} \%$ ), $\mathrm{K}_{2} \mathrm{CO}_{3}$ (38.7 mg, $0.28 \mathrm{mmol}, 2.8$ equiv), 3-(methoxy)phenylboroxine (32 mg, $0.080 \mathrm{mmol}, 0.80$ equiv), and 3q (51 mg, $0.10 \mathrm{mmol}, 1.0$ equiv). Anhydrous acetonitrile ( $0.3 \mathrm{~mL}, 0.33 \mathrm{M})$ was added to the vial. The vial was then equipped with a micro stir bar, capped with a Teflon-coated cap, and removed from the glovebox. The resulting reaction mixture was stirred vigorously at $70{ }^{\circ} \mathrm{C}$ for 24 h. The mixture was then diluted with $\mathrm{Et}_{2} \mathrm{O}$ (approx. $1.5 \mathrm{~mL}$ ) and filtered through a short plug of silica gel. The filter cake was washed with $\mathrm{Et}_{2} \mathrm{O}(5 \times 1 \mathrm{~mL})$, and the resulting solution was concentrated. The yield of the ring-opened product $\mathbf{2 8}^{11}$ was determined to be $56 \%$ by ${ }^{1} \mathrm{H}-\mathrm{NMR}$ analysis with 1,3,5-trimethoxybenzene as an internal standard. No cyclopropyl product 29 was observed. 


\section{Radical Trap Experiment}

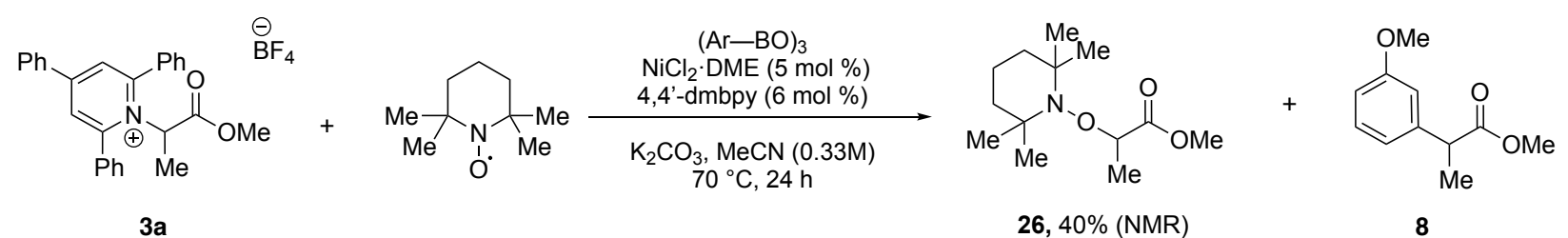

In a $\mathrm{N}_{2}$-filled glovebox: To an oven-dried 1-dram vial was added $\mathrm{NiCl}_{2} \cdot \mathrm{DME}(1.1 \mathrm{mg}, 0.0050$ mmol, 5 mol \%), 4,4'-dimethyl-2,2'-dipyridyl (4,4'-dmbpy, $1.1 \mathrm{mg}, 0.0060 \mathrm{mmol}, 6 \mathrm{~mol} \%$ ), $\mathrm{K}_{2} \mathrm{CO}_{3}$ (38.7 mg, $0.28 \mathrm{mmol}, 2.8$ equiv), 3-(methoxy)phenylboroxine (32 $\mathrm{mg}, 0.080 \mathrm{mmol}, 0.80$ equiv), 2,2,6,6,-tetramethylpiperidine-n-oxyl (TEMPO; $31 \mathrm{mg}, 0.20 \mathrm{mmol}, 2.0$ equiv), and 3a (48 $\mathrm{mg}, 0.10 \mathrm{mmol}, 1.0$ equiv). Anhydrous acetonitrile $(0.3 \mathrm{~mL}, 0.33 \mathrm{M})$ was added to the vial. The vial was then equipped with a micro stir bar, capped with a Teflon-coated cap, and removed from the glovebox. The resulting reaction mixture was stirred vigorously at $70^{\circ} \mathrm{C}$ for $24 \mathrm{~h}$. The mixture was then diluted with $\mathrm{Et}_{2} \mathrm{O}$ (approx. $1.5 \mathrm{~mL}$ ) and filtered through a short plug of silica gel. The filter cake was washed with $\mathrm{Et}_{2} \mathrm{O}(5 \times 1 \mathrm{~mL})$, and the resulting solution was concentrated. The yield of the known TEMPO adduct ${ }^{12}$ (26) was determined to be $40 \%$ by ${ }^{1} \mathrm{H}-\mathrm{NMR}$ analysis with 1,3,5-trimethoxybenzene as an internal standard. No cross-coupled product 8 was observed.

\section{References}

1. Pangborn, A. B.; Giardello, M. A.; Grubbs, R. H.; Rosen, R. K.; Timmers, F. J., Safe and convenient procedure for solvent purification. Organometallics 1996, 15 (5), 1518-1520.

2. Rengasamy, R.; Curtis-Long, M. J.; Seo, W. D.; Jeong, S. H.; Jeong, I. Y.; Park, K. H., New building block for polyhydroxylated piperidine: Total synthesis of 1,6-dideoxynojirimycin. J. Org. Chem. 2008, 73 (7), 2898-2901.

3. Brindisi, M.; Ulivieri, C.; Alfano, G.; Gemma, S.; de Asís Balaguer, F.; Khan, T.; Grillo, A.; Chemi, G.; Menchon, G.; Prota, A. E.; Olieric, N.; Lucena-Agell, D.; Barasoain, I.; Diaz, J. F.; Nebbioso, A.; Conte, M.; Lopresti, L.; Magnano, S.; Amet, R.; Kinsella, P.; Zisterer, D. M.; Ibrahim, O.; O'Sullivan, J.; Morbidelli, L.; Spaccapelo, R.; Baldari, C.; Butini, S.; Novellino, E.; Campiani, G.; Altucci, L.; Steinmetz, M. O.; Brogi, S., Structure-activity relationships, biological evaluation and structural studies of novel pyrrolonaphthoxazepines as antitumor agents. Eur. J. Med. Chem. 2019, 162, 290-320.

4. Gehrtz, P. H.; Hirschbeck, V.; Fleischer, I., A recyclable CO surrogate in regioselective alkoxycarbonylation of alkenes: indirect use of carbon dioxide. Chem. Commun. 2015, 51 (63), 12574-12577.

5. Williams, C. M.; Johnson, J. B.; Rovis, T., Nickel-Catalyzed Reductive Carboxylation of Styrenes Using CO2. J. Am. Chem. Soc. 2008, 130 (45), 14936-14937. 
6. Nishiguchi, I.; Sunderrao, K. P.; Yamamoto, U.; Yamamoto, Y.; Uchida, T.; Maekawa, H., One-pot vicinal and geminal double carboalkoxylation with N-carboalkoxy-imidazole by electroreduction of aromatic vinyl and imine derivatives. Electrochemistry 2006, 74 (8), 680-684.

7. Basch, C. H.; Liao, J.; Xu, J.; Piane, J. J.; Watson, M. P., Harnessing Alkyl Amines as Electrophiles for Nickel-Catalyzed Cross Couplings via C-N Bond Activation. J. Am. Chem. Soc. 2017, 139 (15), 5313-5316.

8. Katritzky, A. R.; Manzo, R. H.; Lloyd, J. M.; Patel, R. C., Mechanism of the Pyrylium/Pyridinium Ring Interconversion. Mild Preparative Conditions for Conversion of Amines into Pyridinium Ions. Angew. Chem., Int. Ed. Engl. 1980, 19 (4), 306-306.

9. Chalker, J. M.; Gunnoo, S. B.; Boutureira, O.; Gerstberger, S. C.; Fernandez-Gonzalez, M.; Bernardes, G. J. L.; Griffin, L.; Hailu, H.; Schofield, C. J.; Davis, B. G., Methods for converting cysteine to dehydroalanine on peptides and proteins. Chem. Sci. 2011, 2 (9), 16661676.

10. Klauck, F. J. R.; James, M. J.; Glorius, F., Deaminative Strategy for the Visible-LightMediated Generation of Alkyl Radicals. Angew. Chem., Int. Ed. 2017, 56 (40), 12336-12339.

11. Nagumo, S.; Miura, T.; Mizukami, M.; Miyoshi, I.; Imai, M.; Kawahara, N.; Akita, H., Intramolecular Friedel-Crafts type reaction of vinyloxiranes linked to an ester group. Tetrahedron 2009, 65 (47), 9884-9896.

12. Fan, J. H.; Wei, W. T.; Zhou, M. B.; Song, R. J.; Li, J. H., Palladium-Catalyzed Oxidative Difunctionalization of Alkenes with alpha-Carbonyl Alkyl Bromides Initiated through a Hecktype Insertion: A Route to Indolin-2-ones. Angew. Chem., Int. Ed. 2014, 53 (26), 6650-6654. 


\section{HPLC Spectra}

Compound 8, racemic and prepared from racemic alanine methyl ester

$\mathrm{mAU}$

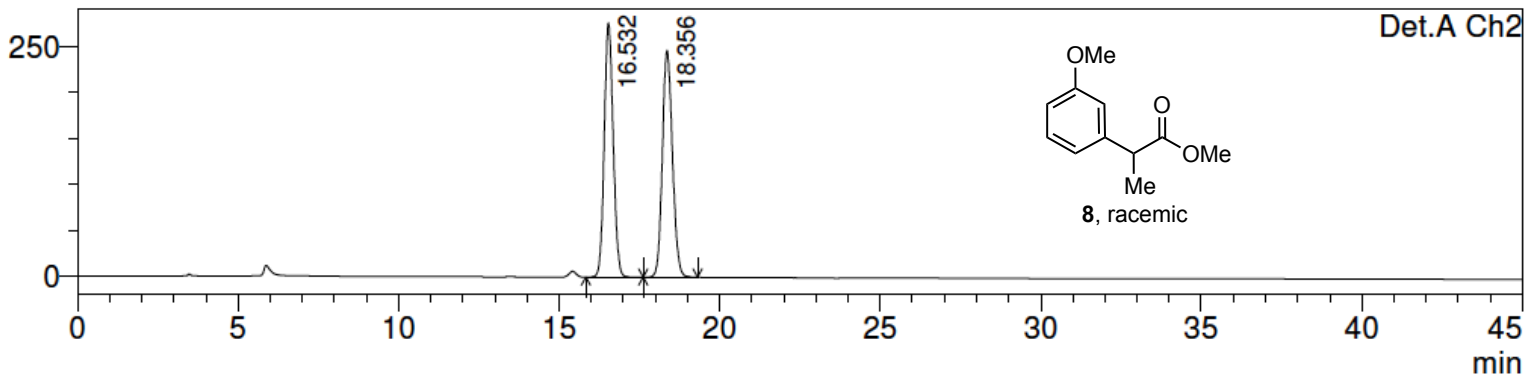

Detector A Ch2 210nm

\begin{tabular}{|r|r|r|r|r|r|}
\hline \multicolumn{1}{|c|}{ Peak\# } & \multicolumn{1}{|c|}{ Ret. Time } & \multicolumn{1}{c|}{ Area } & \multicolumn{1}{c|}{ Height } & \multicolumn{1}{c|}{ Area \% } & \multicolumn{1}{c|}{ Height \% } \\
\hline 1 & 16.532 & 5377219 & 276748 & 49.928 & 52.851 \\
\hline 2 & 18.356 & 5392653 & 246889 & 50.072 & 47.149 \\
\hline Total & & 10769872 & 523637 & 100.000 & 100.000 \\
\hline
\end{tabular}

Compound 8, racemic and prepared from enantiopure alanine methyl ester

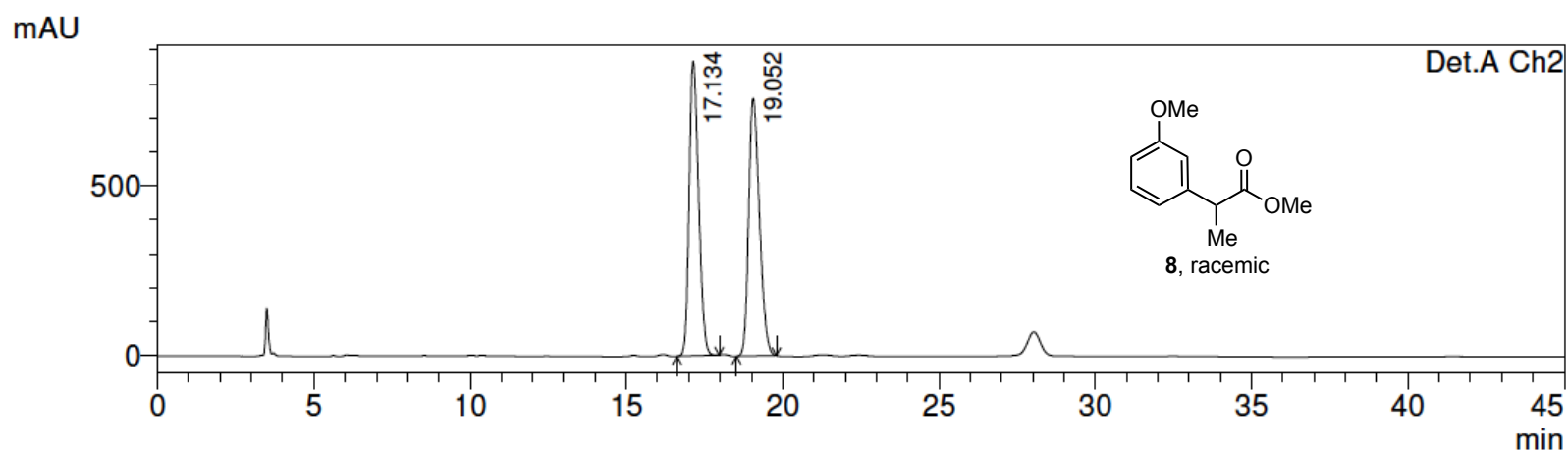

Detector A Ch2 210nm

\begin{tabular}{|r|r|r|r|r|r|}
\hline \multicolumn{1}{|c|}{ Peak\# } & Ret. Time & \multicolumn{1}{|c|}{ Area } & \multicolumn{1}{c|}{ Height } & \multicolumn{1}{c|}{ Area \% } & \multicolumn{1}{c|}{ Height \% } \\
\hline 1 & 17.134 & 18157377 & 867082 & 49.919 & 53.330 \\
\hline 2 & 19.052 & 18216638 & 758784 & 50.081 & 46.670 \\
\hline Total & & 36374015 & 1625866 & 100.000 & 100.000 \\
\hline
\end{tabular}


Compound 7

${ }^{1} \mathrm{H}$ NMR (600 MHz, $\mathrm{CDCl}_{3}$ )

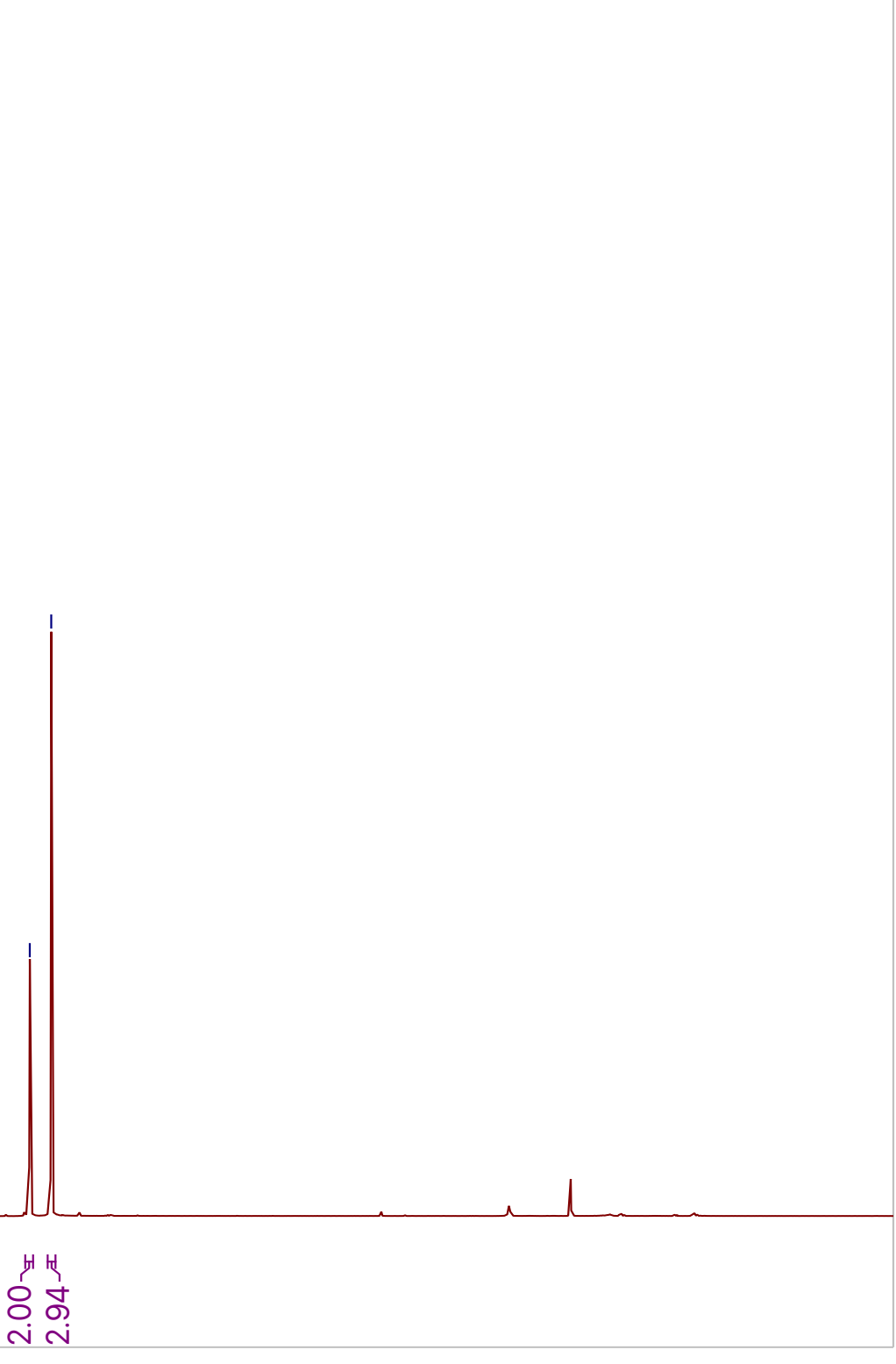




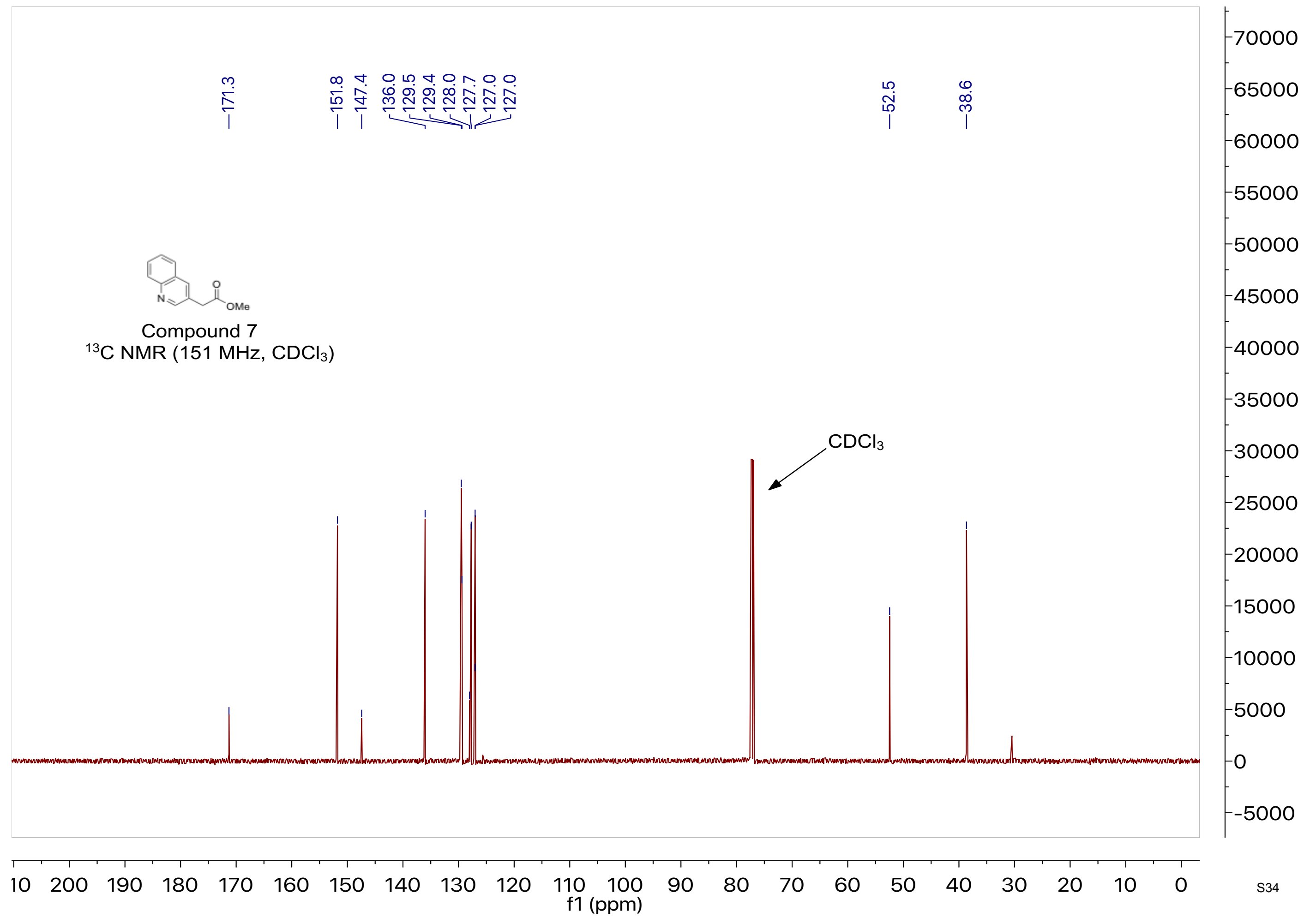




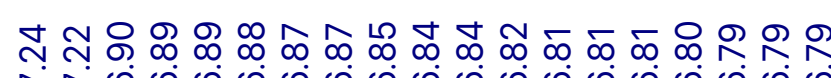

ヘ

作

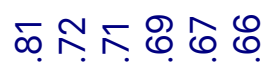

लं $\dot{m} \dot{m} \dot{m} \dot{m}$

急

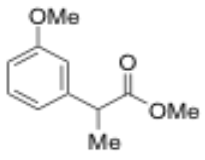

Compound 8

${ }^{1} \mathrm{H}$ NMR (400 MHz, $\left.\mathrm{CDCl}_{3}\right)$
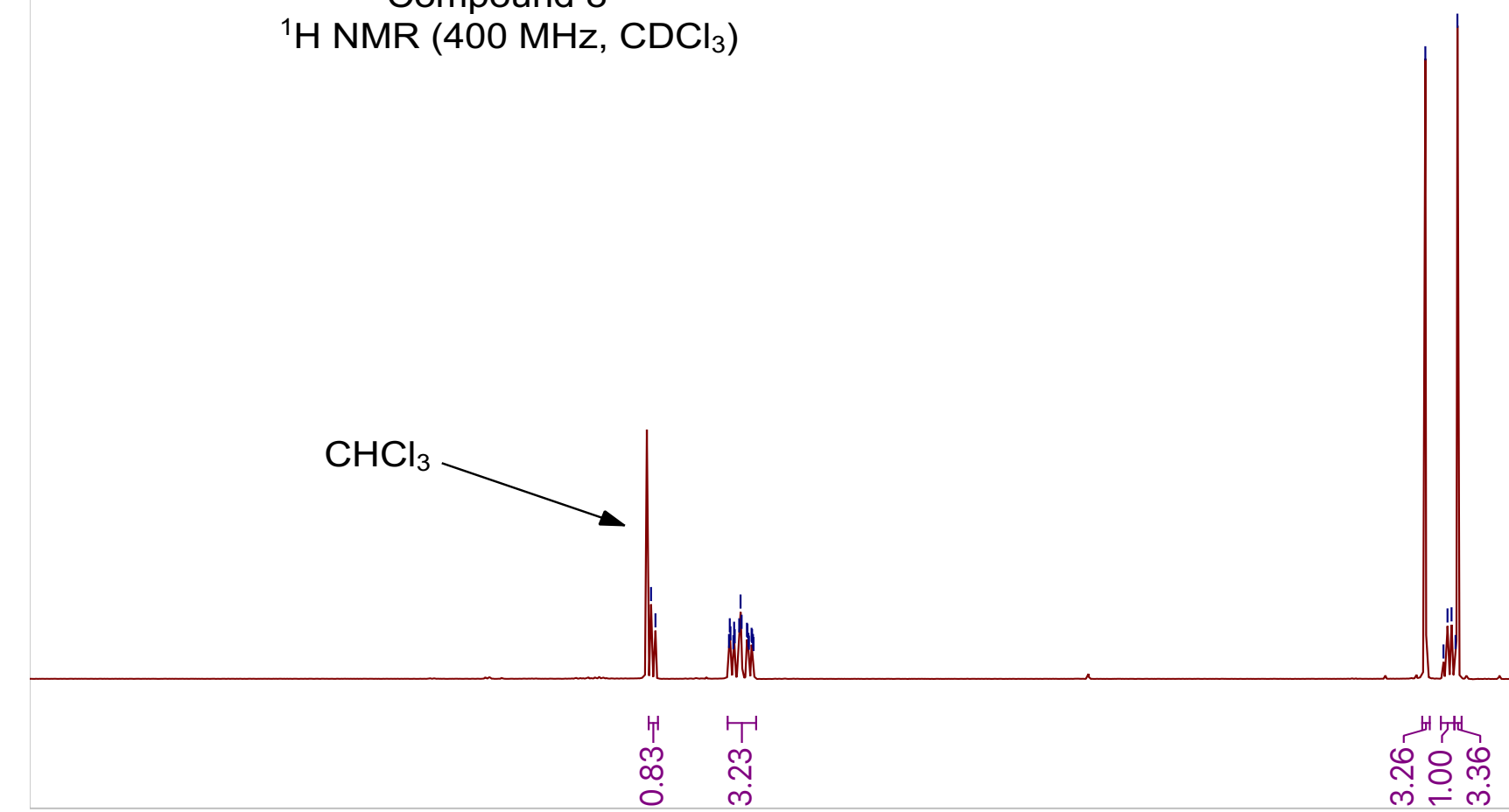


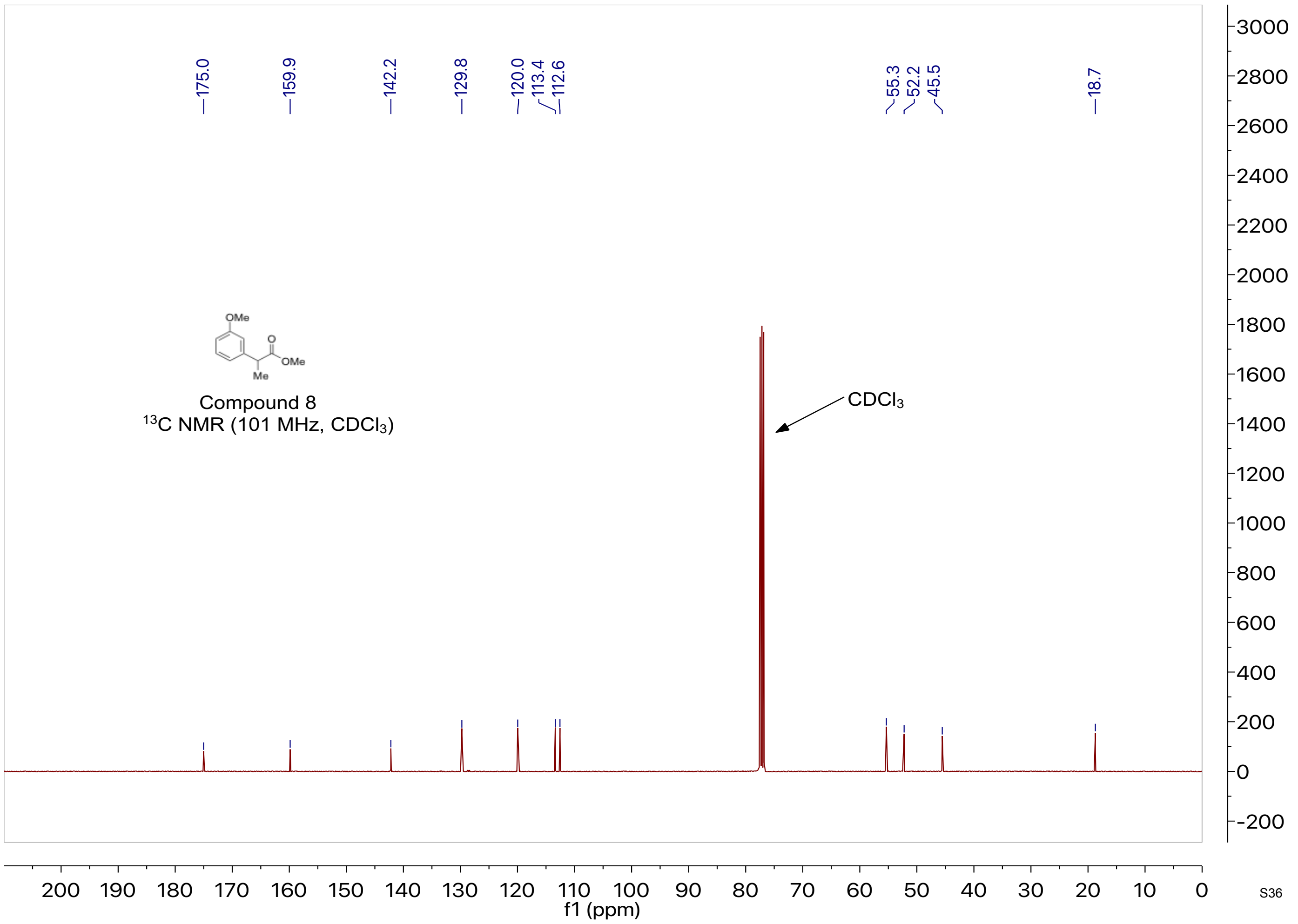




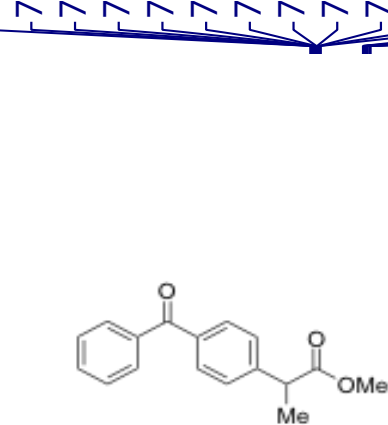

Compound 9

${ }^{1} \mathrm{H}$ NMR $\left(600 \mathrm{MHz}, \mathrm{CDCl}_{3}\right)$ 


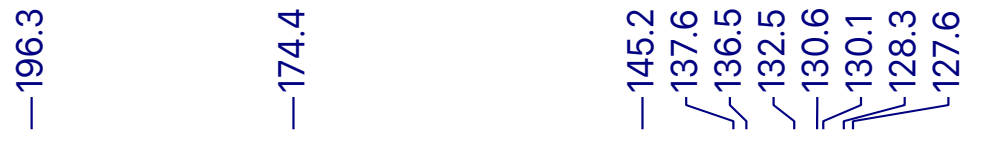

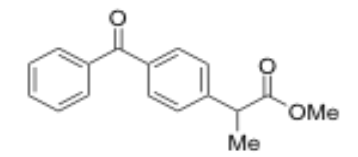

Compound 9

${ }^{13} \mathrm{C}$ NMR $\left(101 \mathrm{MHz}, \mathrm{CDCl}_{3}\right)$

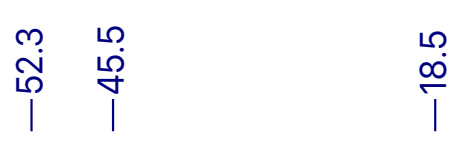



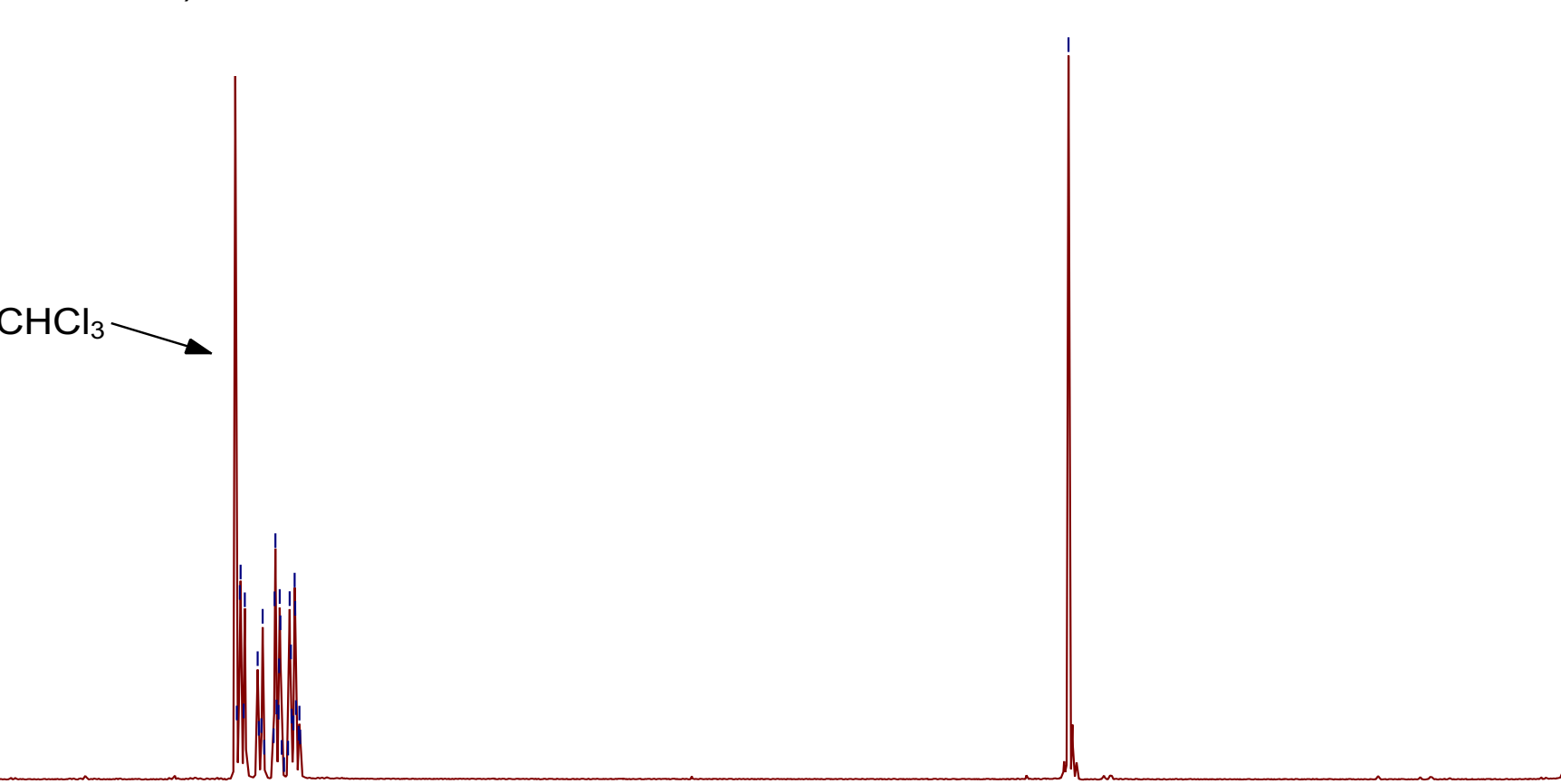

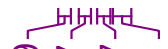

옹ㅇㅇㅇㅛ 

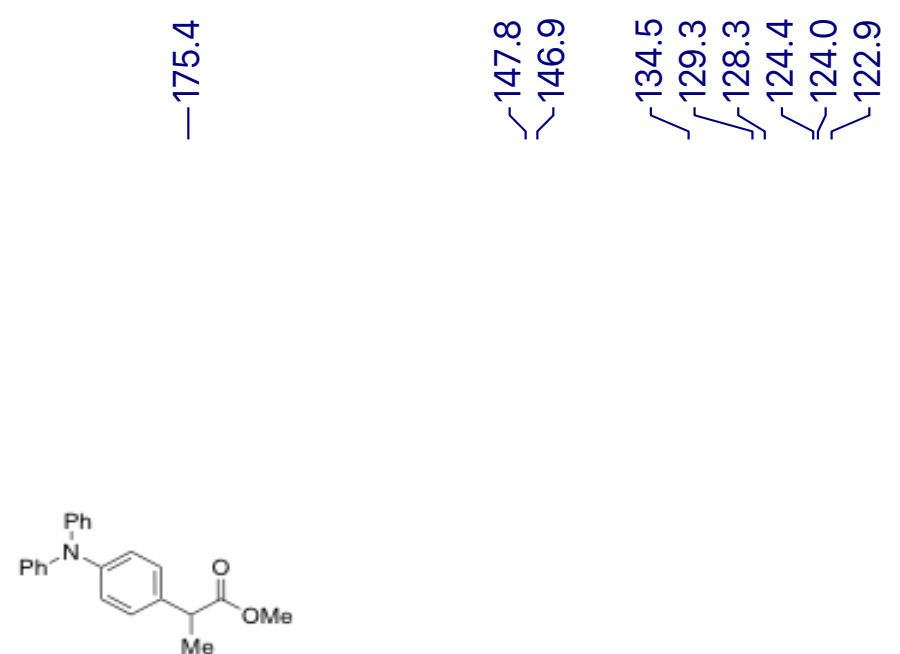

Compound 10

${ }^{13} \mathrm{C}$ NMR $\left(101 \mathrm{MHz}, \mathrm{CDCl}_{3}\right)$

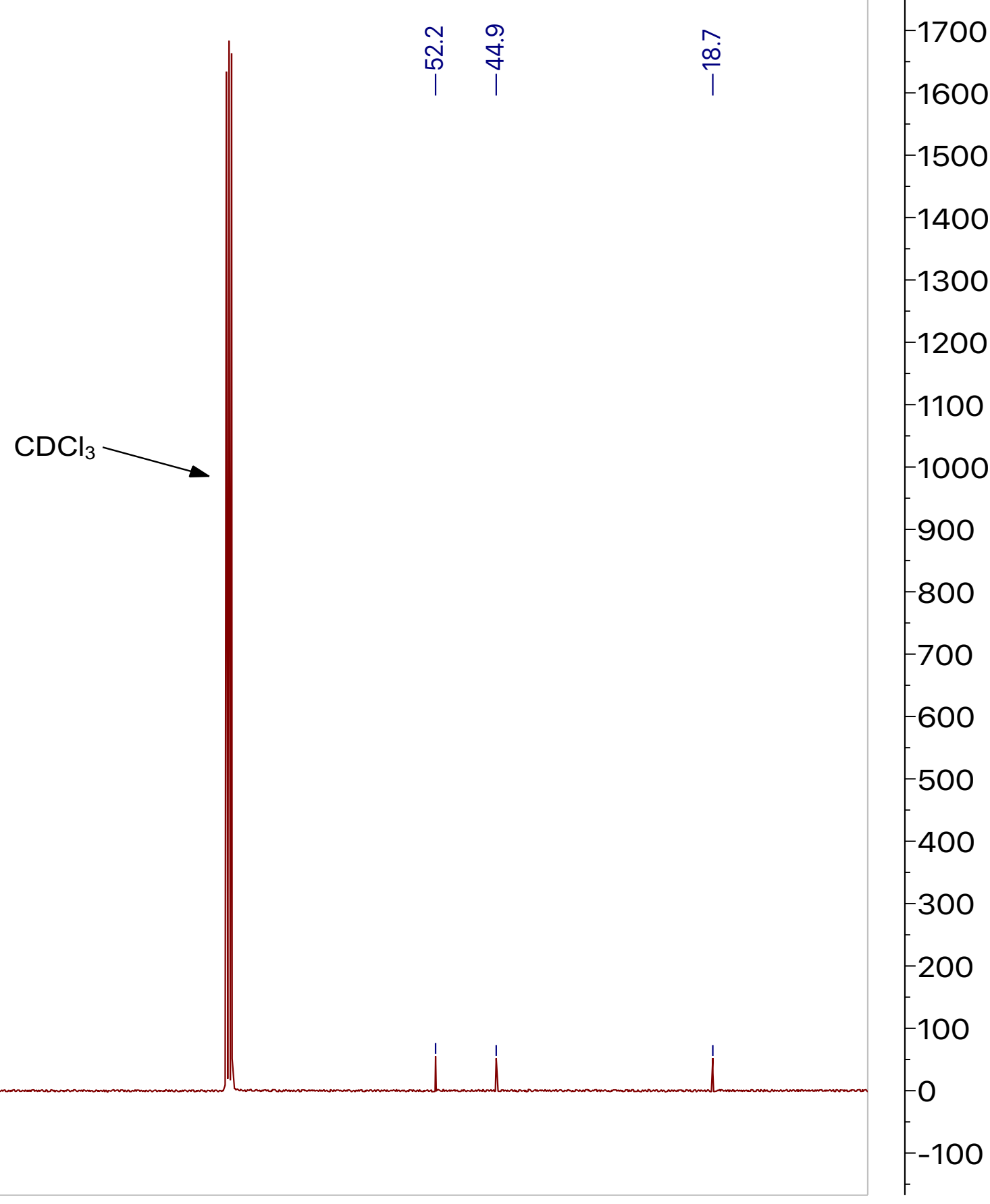




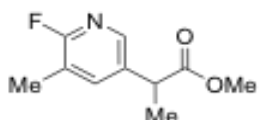

Compound 11

${ }^{1} \mathrm{H}$ NMR $\left(600 \mathrm{MHz}, \mathrm{CDCl}_{3}\right)$

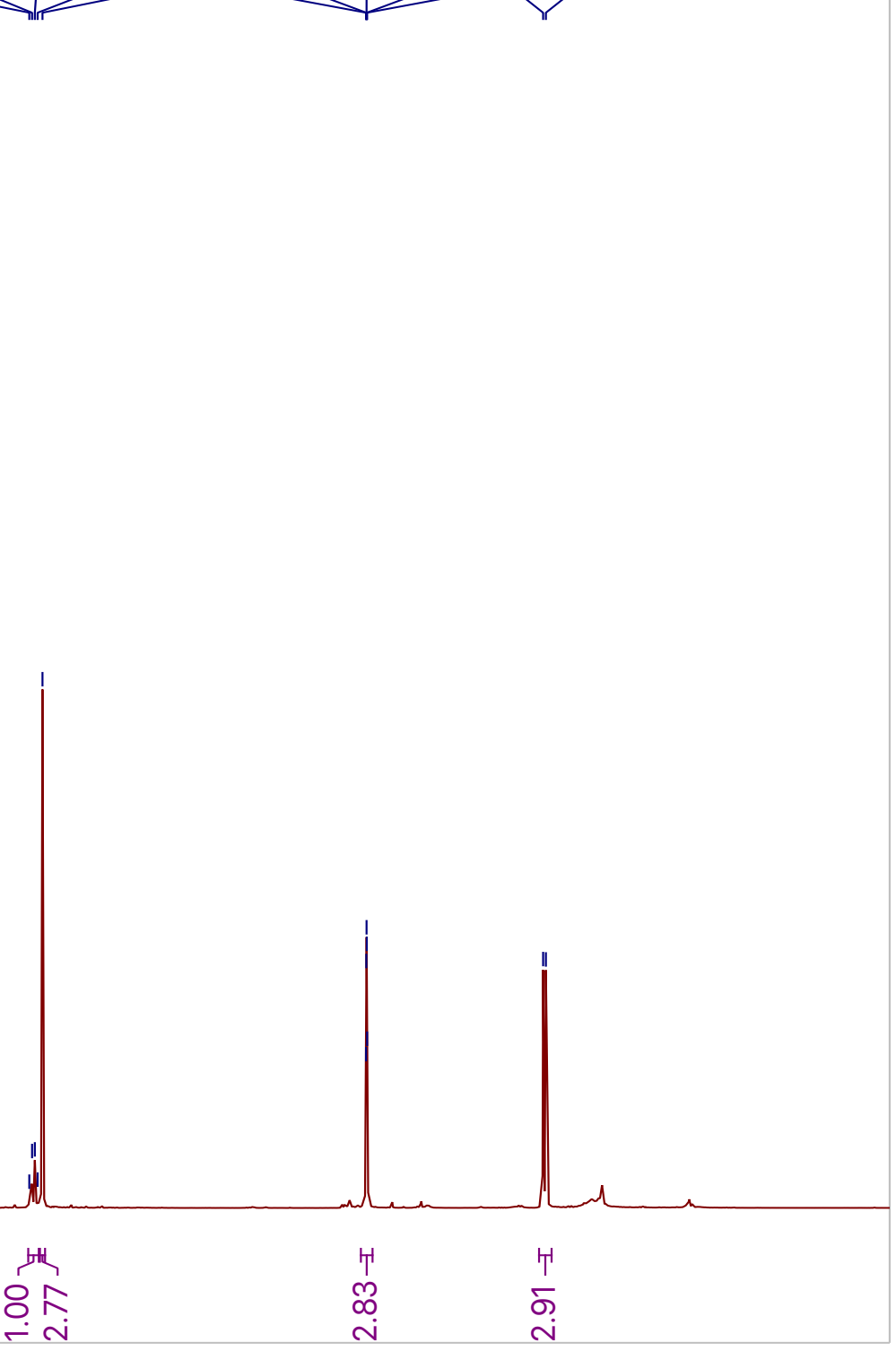




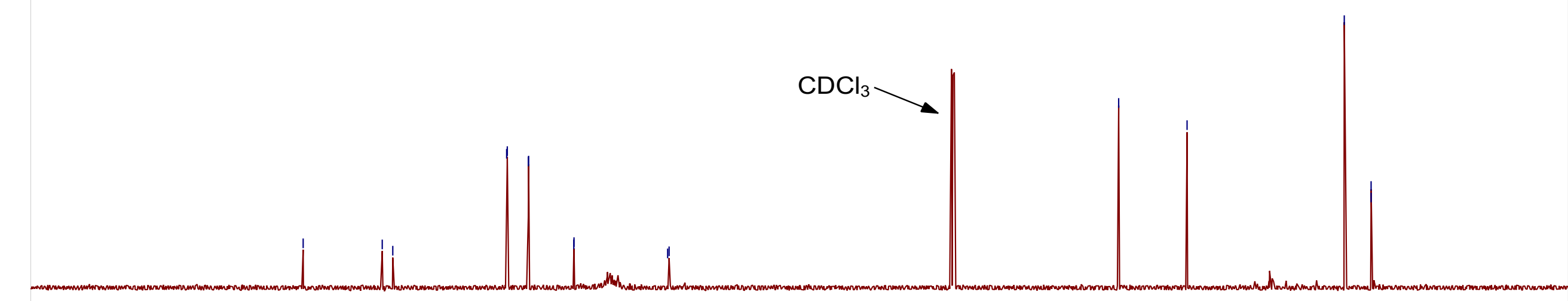


Compound 11 $\left.{ }^{19} \mathrm{~F} \mathrm{NMR} \mathrm{(565} \mathrm{MHz,} \mathrm{CDCl}_{3}\right)$ 


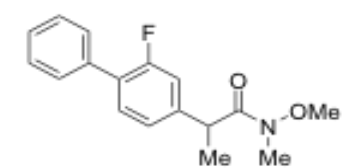

9000

Compound 12

${ }^{1} \mathrm{H}$ NMR $\left(600 \mathrm{MHz}, \mathrm{CDCl}_{3}\right)$

-8000
-7000
-6000
-5000
-4000
-3000
-2000
-1000
-0
-1000




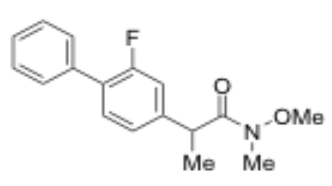

Compound 12 ${ }^{13} \mathrm{C}$ NMR $\left(101 \mathrm{MHz}, \mathrm{CDCl}_{3}\right)$

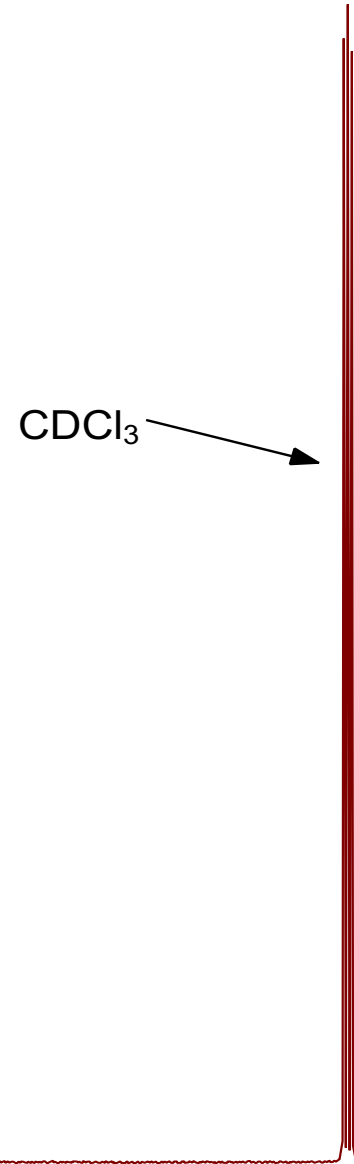

$-1600$

$-1500$

$-1400$

$-1300$

$-1200$

$-1100$

$-1000$

$-900$

$-800$

$-700$

$-600$

$-500$

$-400$

$-300$

$-200$

$-100$

$-0$

$-100$

$-200$ 


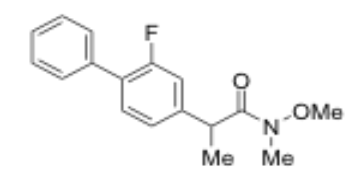

Compound 12

${ }^{19} \mathrm{~F}$ NMR (565 MHz, $\mathrm{CDCl}_{3}$ )

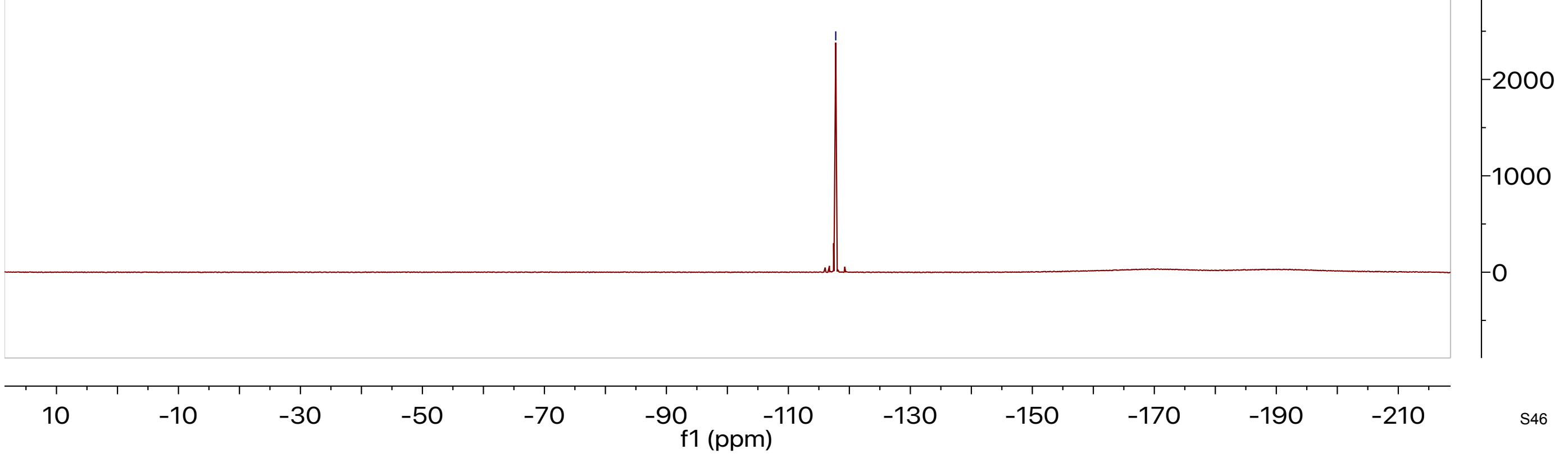




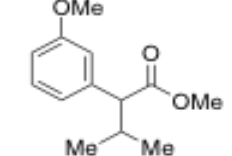

Compound 13

${ }^{1} \mathrm{H}$ NMR (600 MHz, $\mathrm{CDCl}_{3}$ )
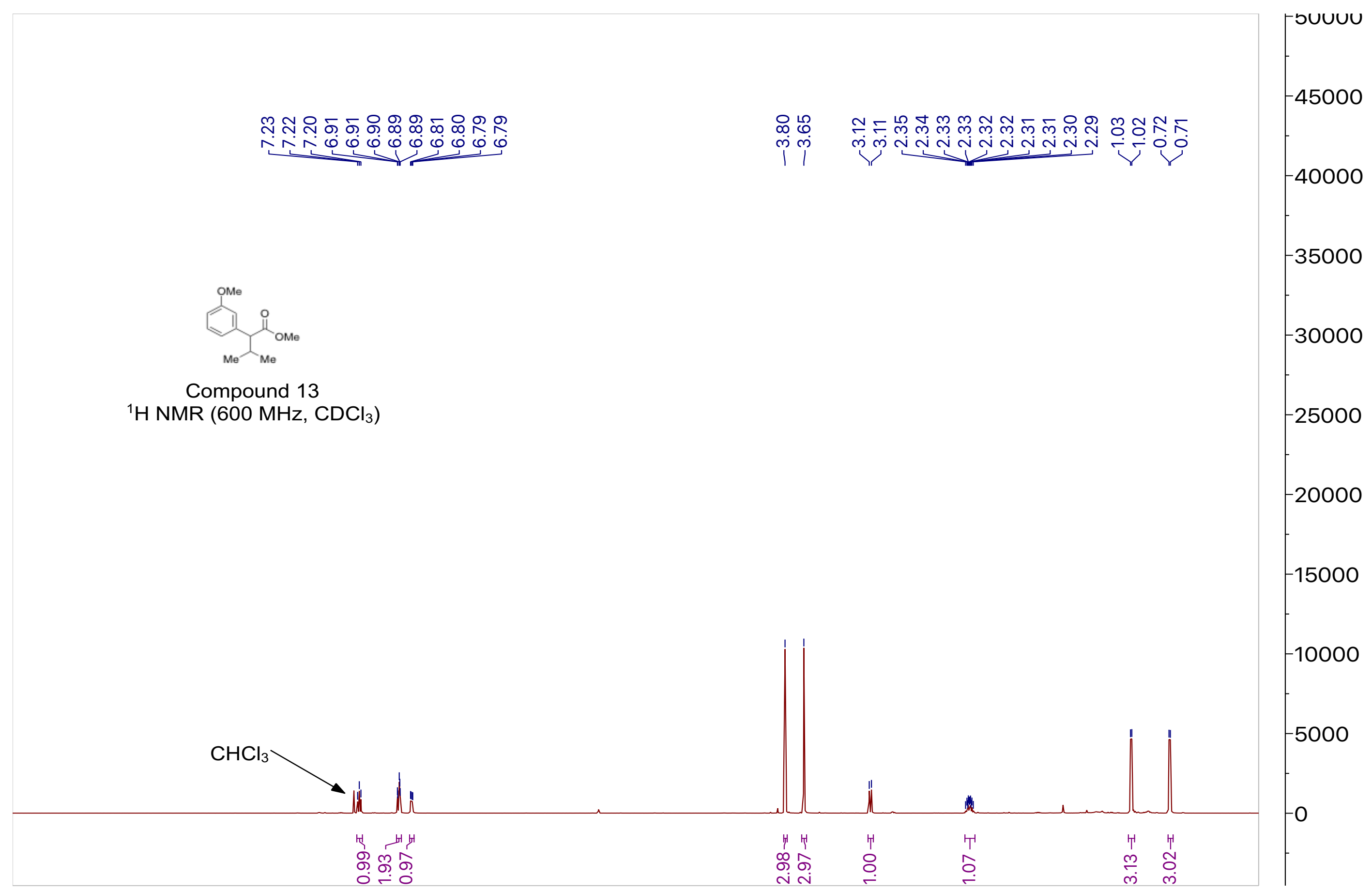


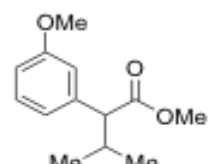

Compound 13

${ }^{13} \mathrm{C}$ NMR $\left(151 \mathrm{MHz}, \mathrm{CDCl}_{3}\right)$

70000

$-60000$

$-50000$

$\mathrm{CDCl}_{3}$

$-40000$

$-30000$

$-20000$

$-10000$

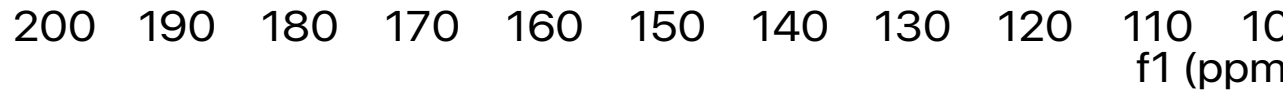




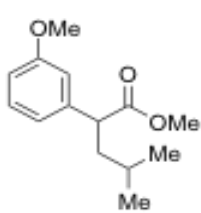

Compound 14

${ }^{1} \mathrm{H} \mathrm{NMR}\left(600 \mathrm{MHz}, \mathrm{CDCl}_{3}\right)$

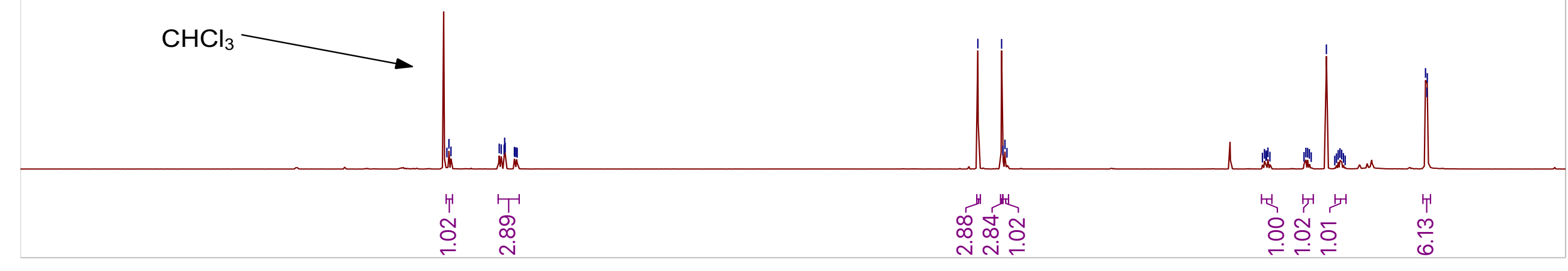

-22000
-21000
-20000
-19000
-18000
-17000
-16000
-15000
-14000
-13000
-12000
-11000
-10000
-9000
-8000
-7000
-6000
-5000
-4000
-3000
-2000
-1000
-0
--1000
--2000




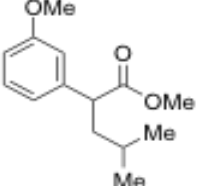

Compound 14

${ }^{13} \mathrm{C}$ NMR (151 MHz, $\mathrm{CDCl}_{3}$ )

5000
50000
45000
40000
35000
30000
25000
20000
5000
10000
5000
5000

$\begin{array}{llllllllllll}0 & 200 & 190 & 180 & 170 & 160 & 150 & 140 & 130 & 120 & 110 & 100\end{array}$

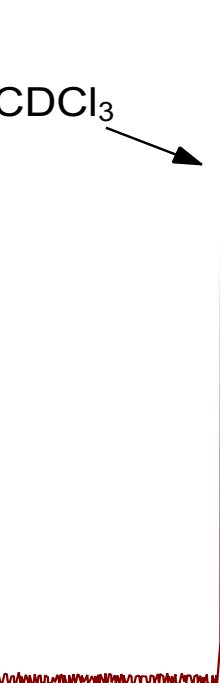




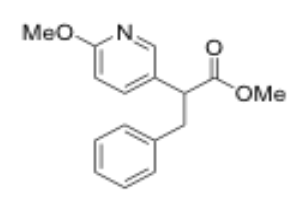

Compound 15

${ }^{1} \mathrm{HNMR}\left(600 \mathrm{MHz}, \mathrm{CDCl}_{3}\right)$

\begin{tabular}{l}
12000 \\
11000 \\
10000 \\
9000 \\
8000 \\
7000 \\
6000 \\
5000 \\
4000 \\
3000 \\
2000 \\
-1000 \\
-1000 \\
\hline
\end{tabular}


Compound 15

${ }^{13} \mathrm{C}$ NMR (151 MHz, $\mathrm{CDCl}_{3}$ )
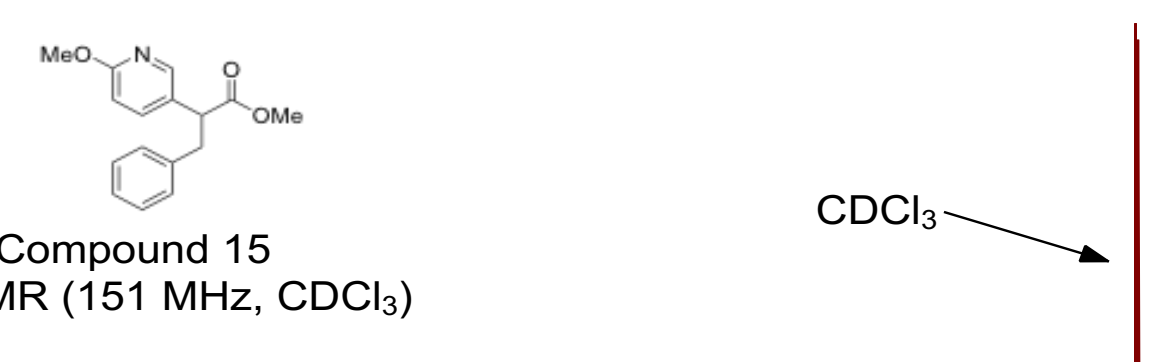


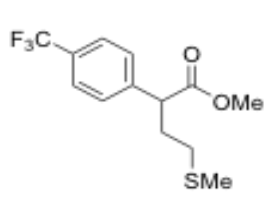

Compound 16 ${ }^{1} \mathrm{H}$ NMR $\left(600 \mathrm{MHz}, \mathrm{CDCl}_{3}\right)$

-22000
-21000
-20000
-19000
-18000
-17000
-16000
-15000
-14000
-13000
-12000
-11000
-10000
-9000
-8000
-7000
-6000
-5000
-4000
-3000
-2000
-1000
-0
--1000
--2000

22000 0000

7000

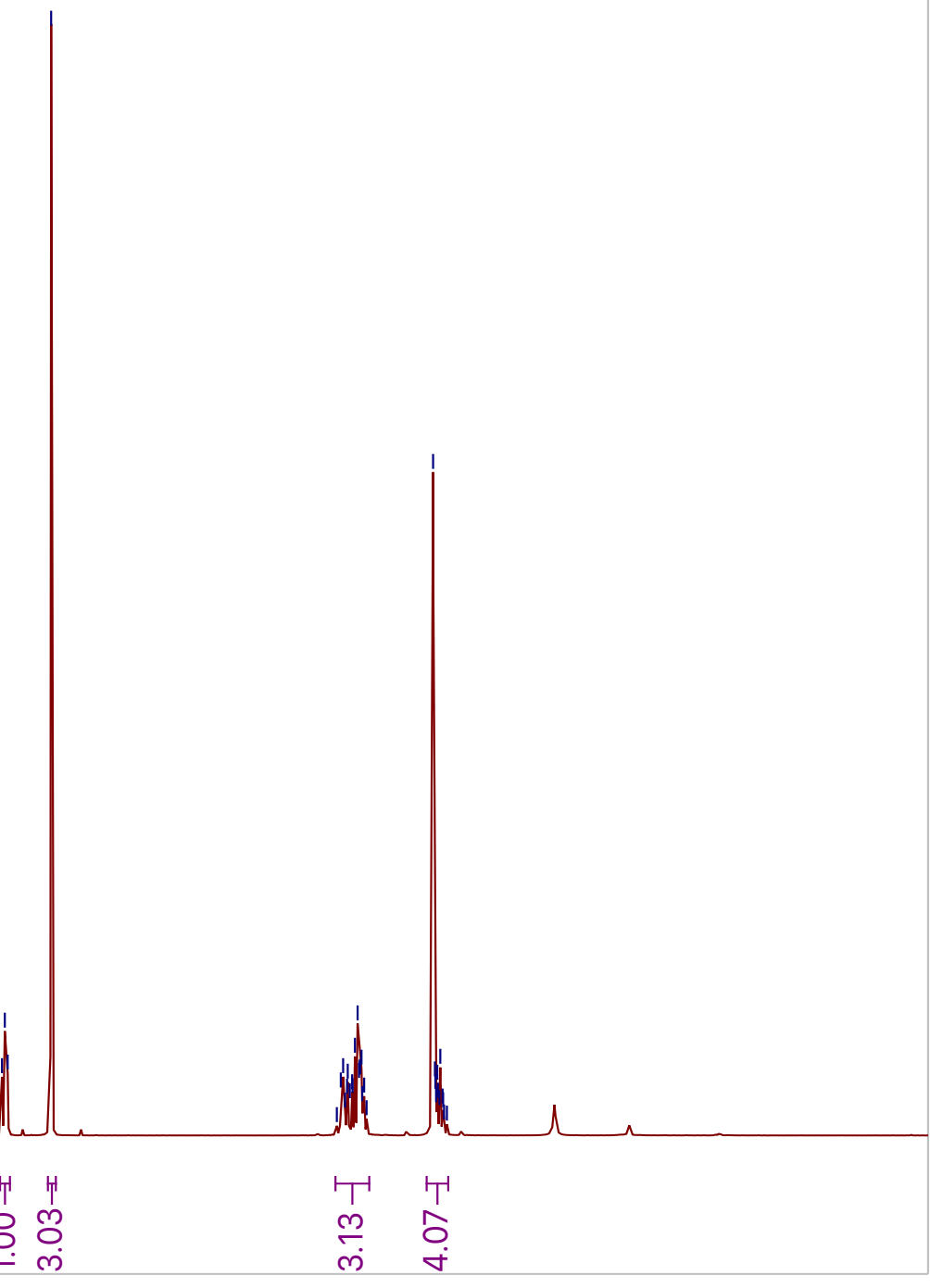

$-2000$ 


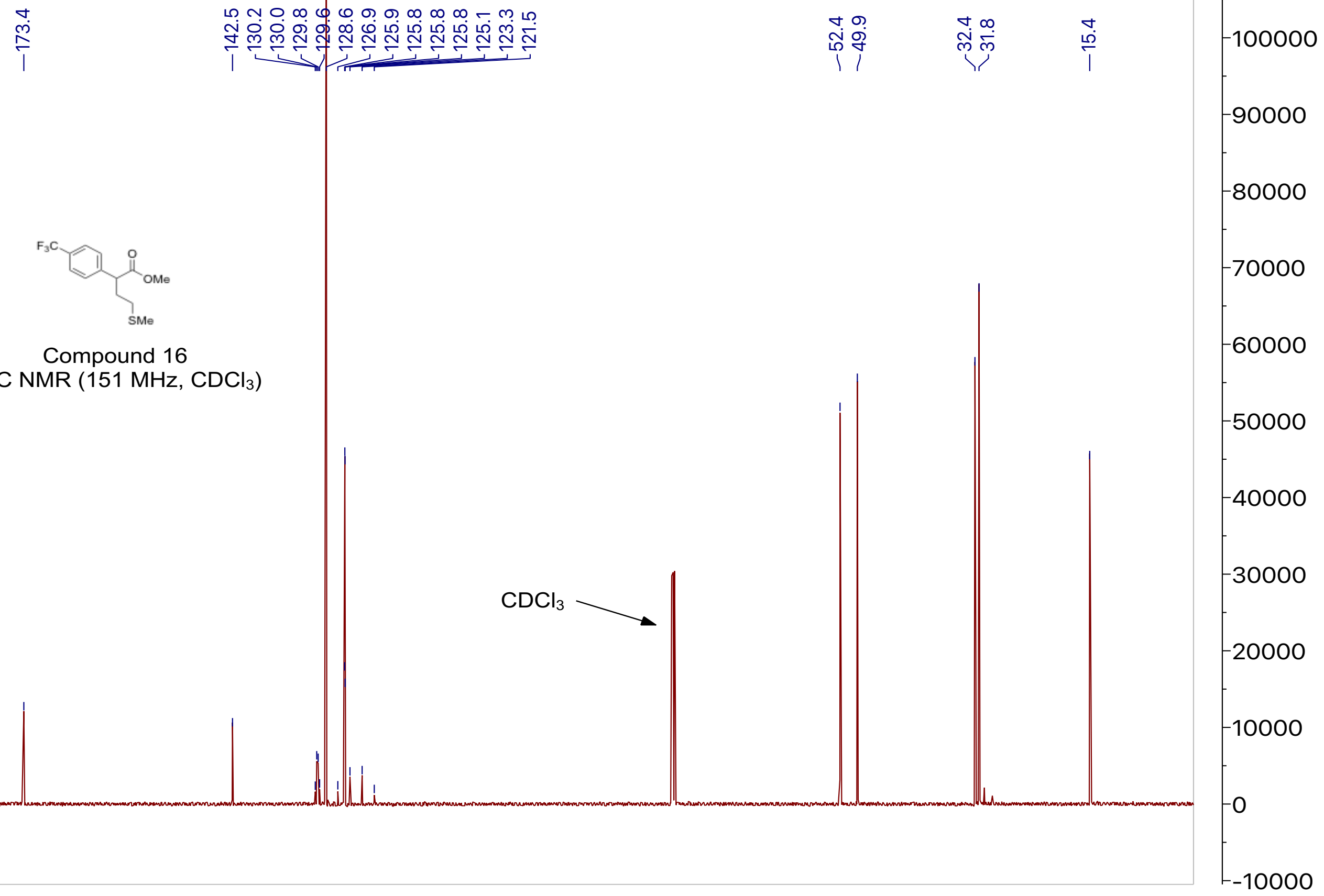

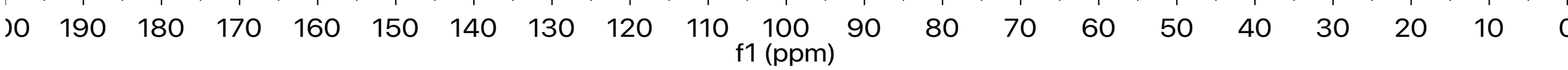




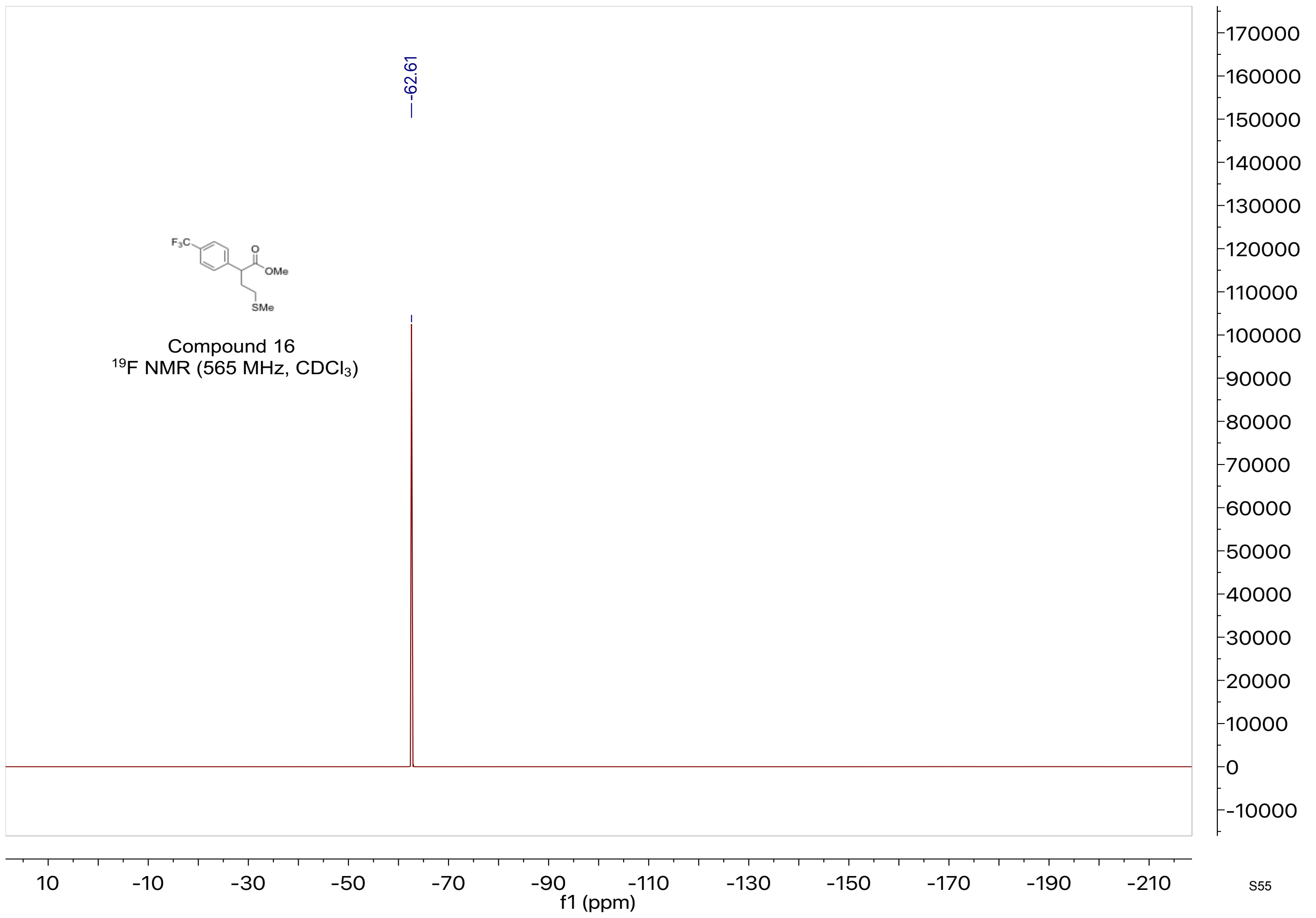




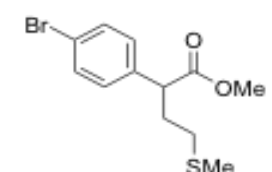

Compound 17

${ }^{1} \mathrm{H}$ NMR $\left(600 \mathrm{MHz}, \mathrm{CDCl}_{3}\right)$

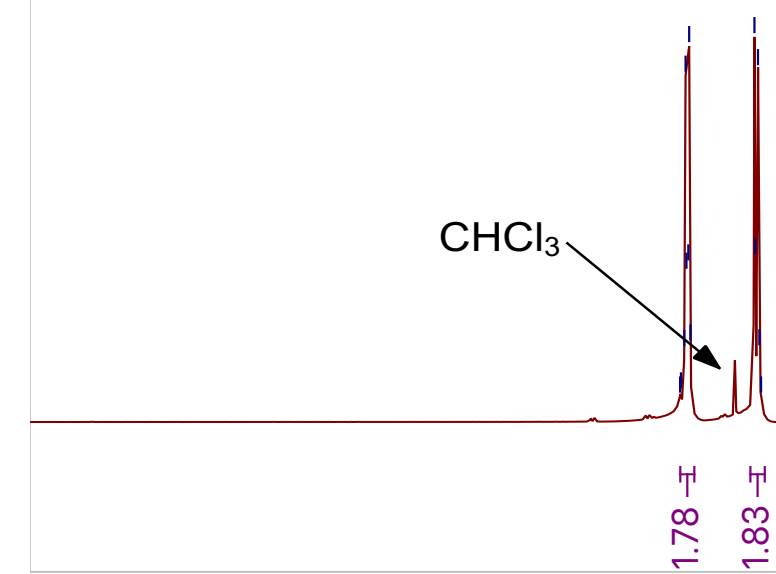

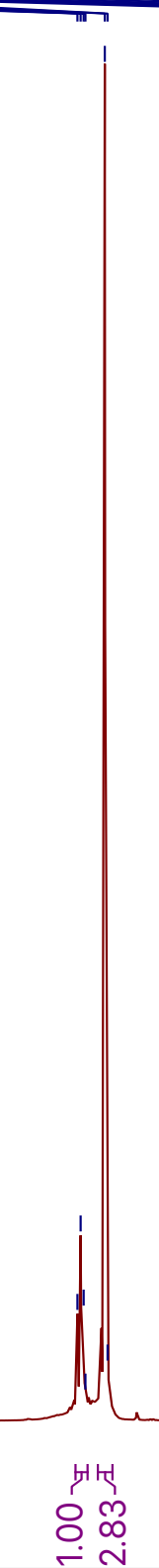

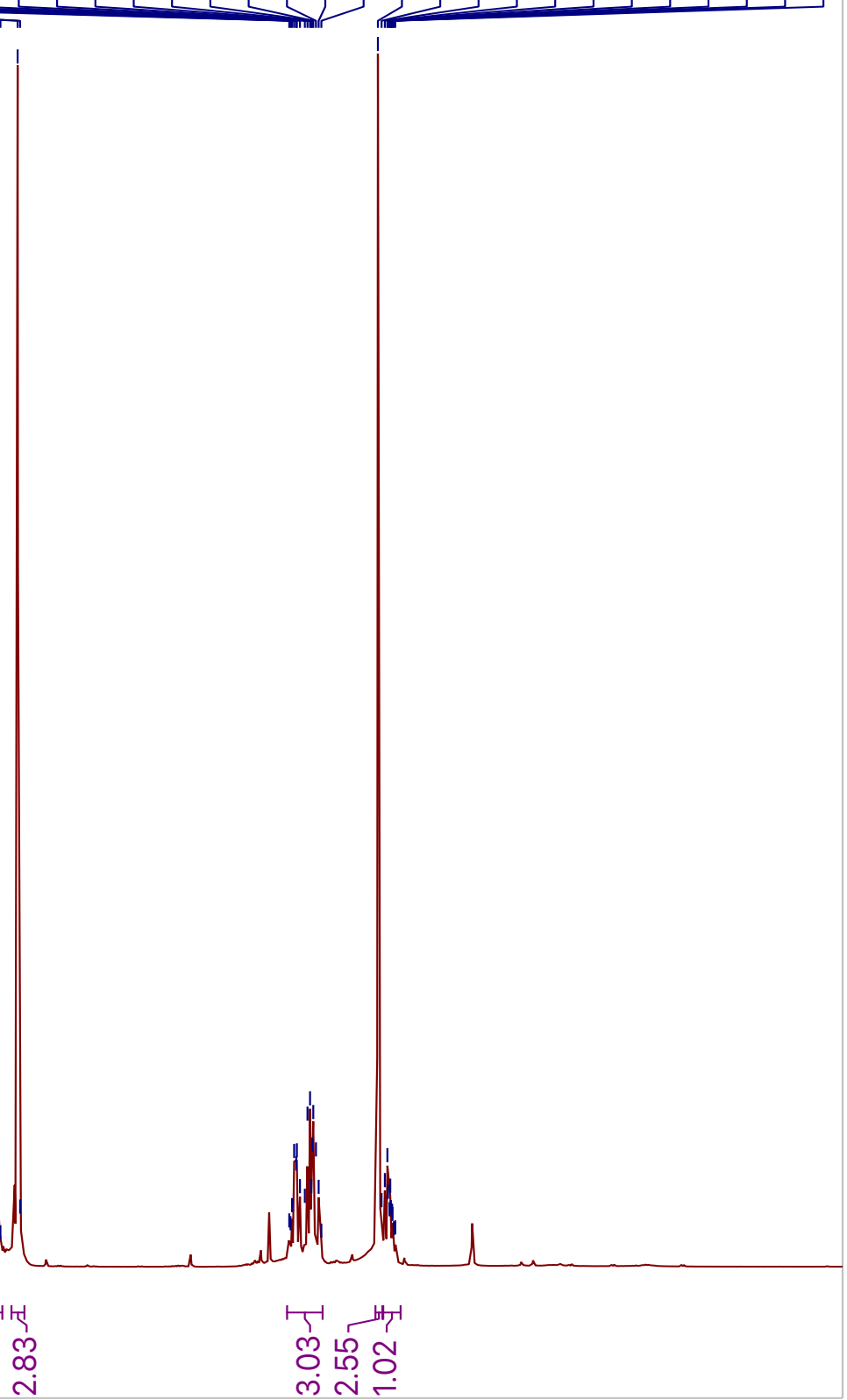

$-16000$

$-15000$

$-14000$

$-13000$

$-12000$

$-11000$

$-10000$

$-9000$

8000

$-7000$

$-6000$

$-5000$

$-4000$

$-3000$

$-2000$

$-1000$

$-0$

$-1000$ 

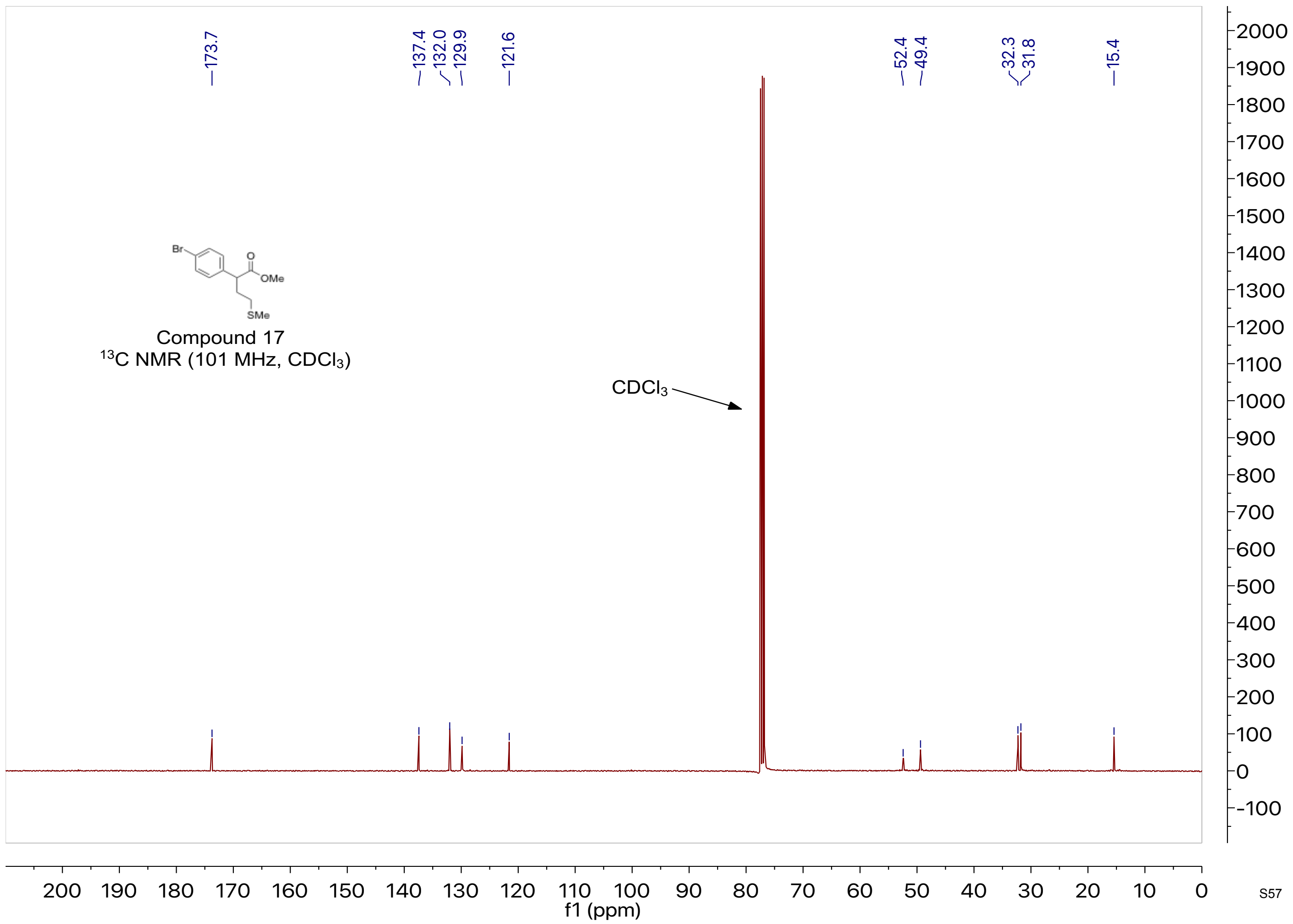

S57 


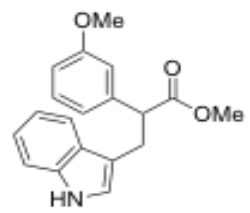

Compound 18

${ }^{1} \mathrm{H}$ NMR $\left(600 \mathrm{MHz}, \mathrm{CDCl}_{3}\right)$

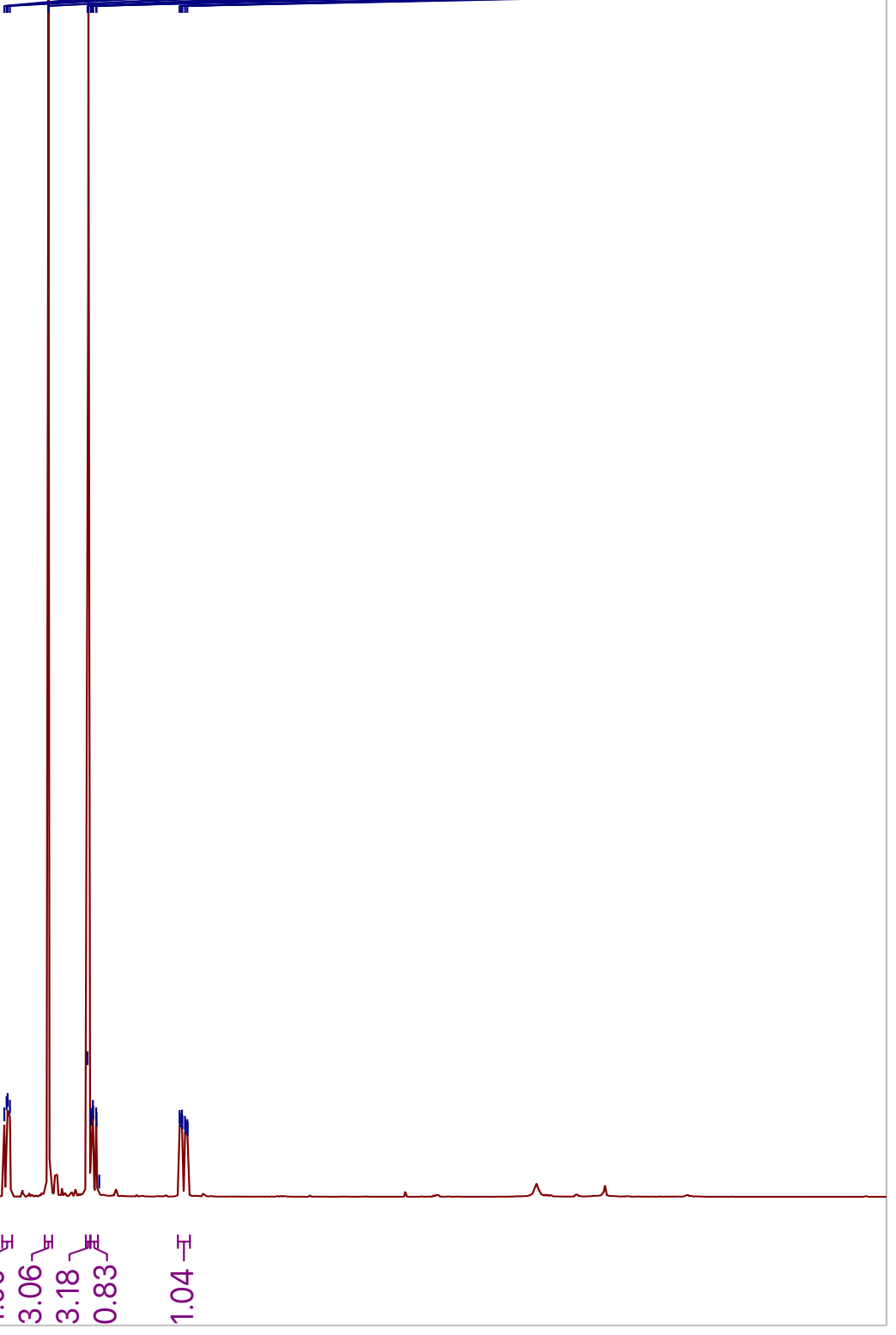

$-9000$

$-8000$

$-7000$

$-6000$

$-5000$

$-4000$

$-3000$

$-2000$

$-1000$

$-0$

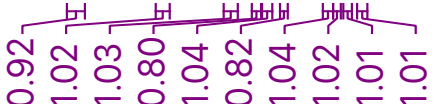

8

$-1000$ 


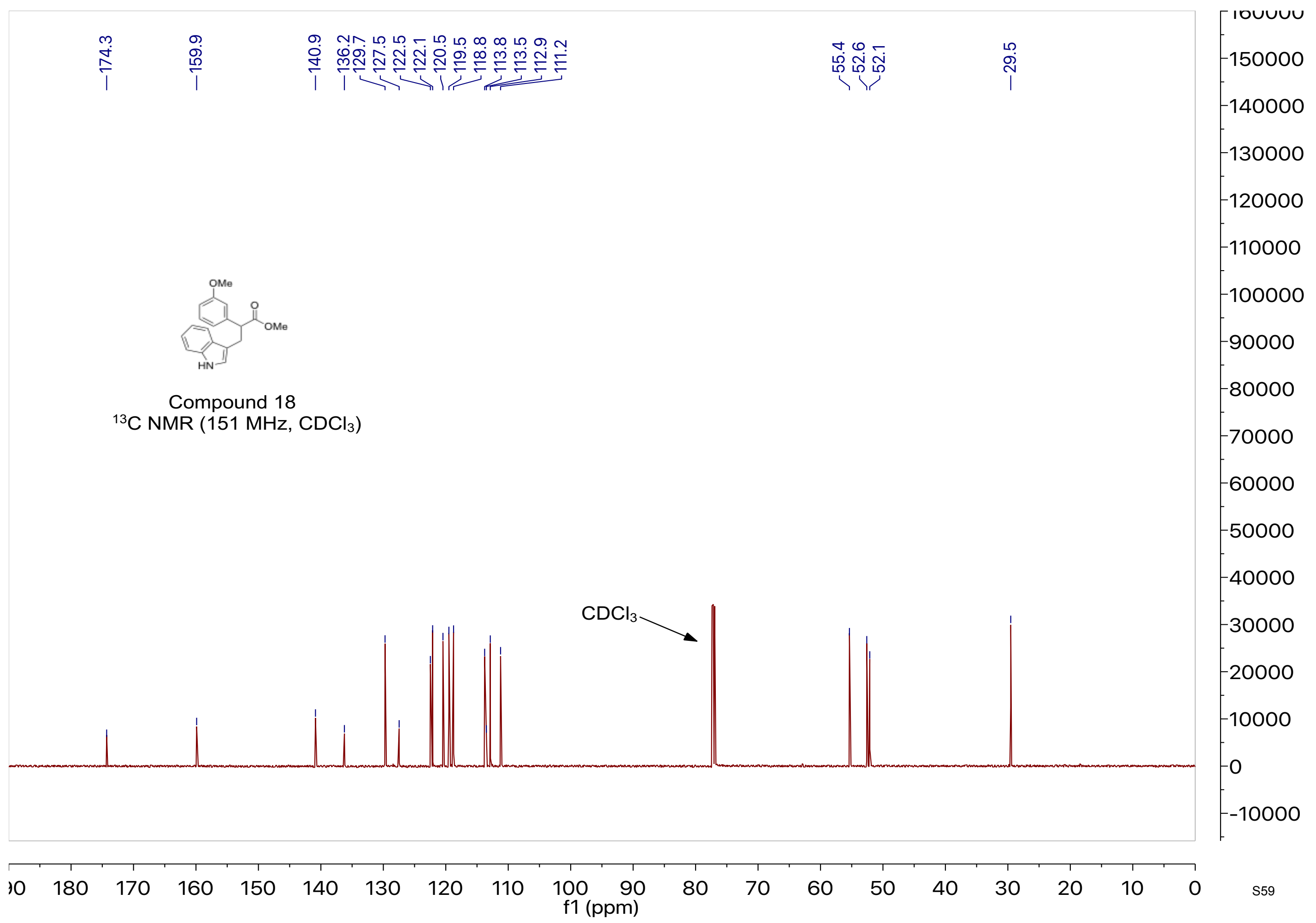




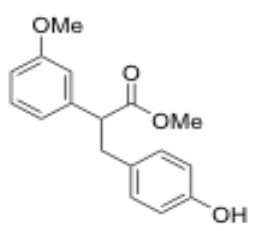

Compound 19

${ }^{1} \mathrm{H}$ NMR $\left(600 \mathrm{MHz}, \mathrm{CDCl}_{3}\right)$

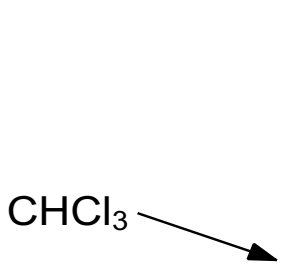




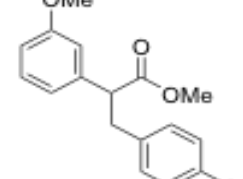

Compound 19

${ }^{13} \mathrm{C}$ NMR $\left(151 \mathrm{MHz}, \mathrm{CDCl}_{3}\right)$

-18000
-16000
-14000
-12000
-10000
-6000
-6000
-4000
-2000
-0
-2000
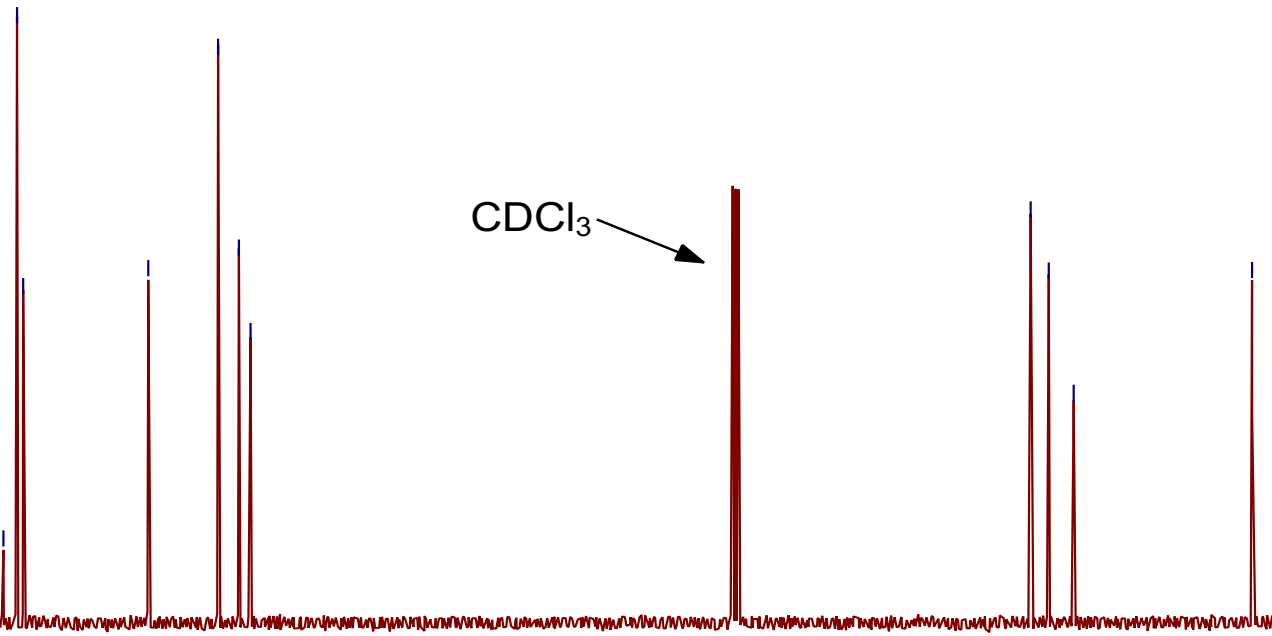

2000

$\begin{array}{lllllllllll}0 & 180 & 170 & 160 & 150 & 140 & 130 & 120 & 110 & 100 & 90\end{array}$

f1 (ppm)

S61 


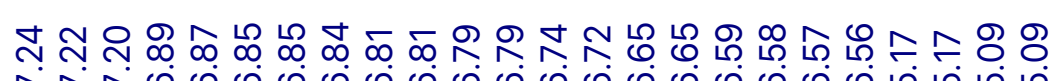
N

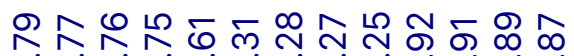

\section{ming}

mmmm

$\mathrm{OMe}$

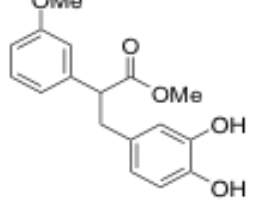

Compound 20

${ }^{1} \mathrm{H}$ NMR (400 MHz, $\mathrm{CDCl}_{3}$ )
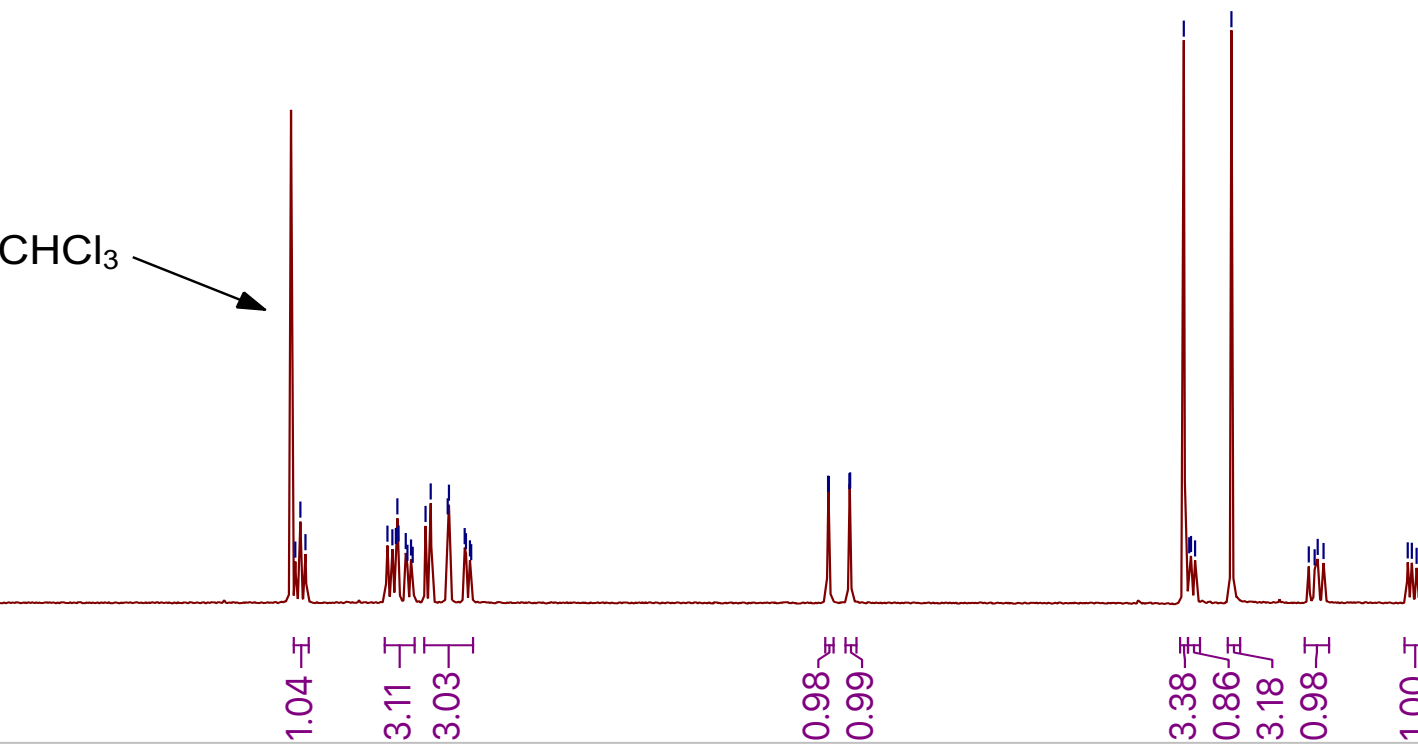


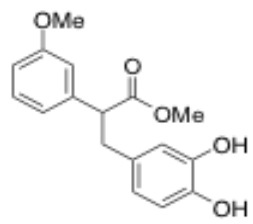

Compound 20

${ }^{13} \mathrm{C}$ NMR (101 MHz, $\mathrm{CDCl}_{3}$ ) 


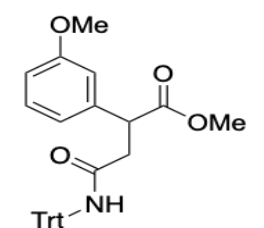

Compound 21

${ }^{13} \mathrm{C}$ NMR $\left(151 \mathrm{MHz}, \mathrm{CDCl}_{3}\right)$

$-22000$

$-20000$

$-18000$

$-16000$

$-14000$

$-12000$

$-10000$

8000

$-6000$

$-4000$

$-2000$

0

$-2000$

J0 $190 \begin{array}{lllllllll}180 & 170 & 160 & 150 & 140 & 130 & 120 & 110 \begin{array}{c}100 \\ \mathrm{f} 1(\mathrm{ppm})\end{array}\end{array}$

$\begin{array}{lllllllll}80 & 70 & 60 & 50 & 40 & 30 & 20 & 10 & 0\end{array}$ 


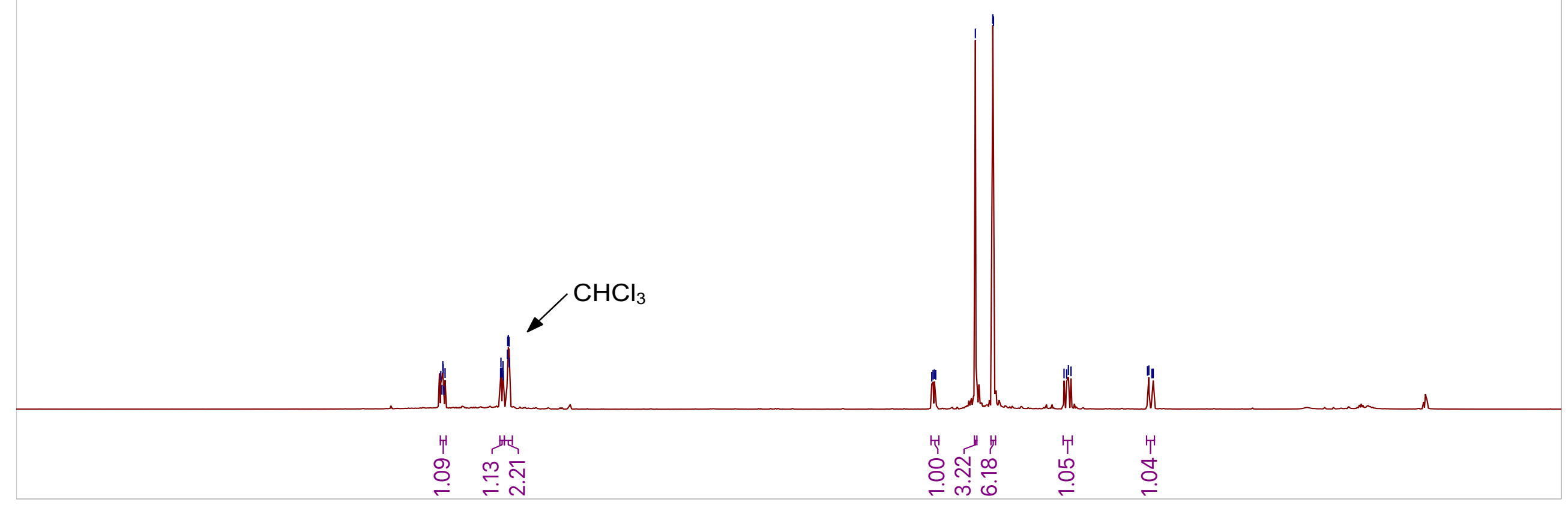




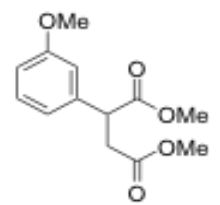

Compound 22

${ }^{13} \mathrm{C}$ NMR $\left(101 \mathrm{MHz}, \mathrm{CDCl}_{3}\right)$

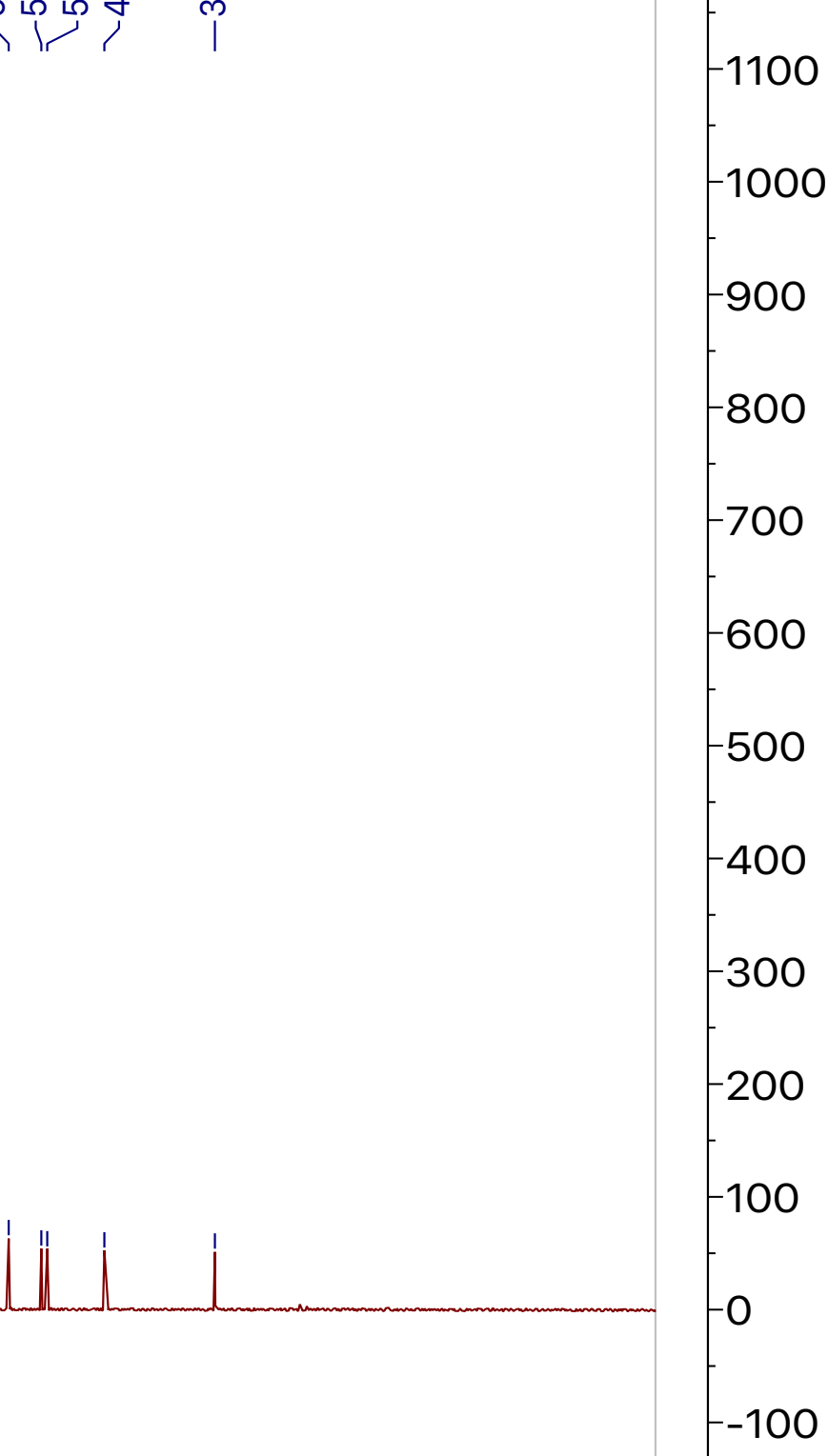

200190180 


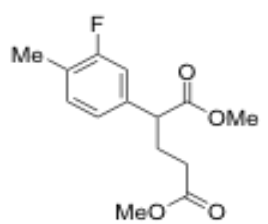

Compound 23

${ }^{1} \mathrm{H}$ NMR $\left(400 \mathrm{MHz}, \mathrm{CDCl}_{3}\right)$

-1200
-1100
-1000
-900
-800
-700
-600
-500
-400
-300
-200
-100
-0
-100




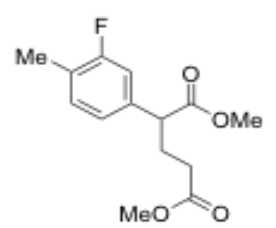

Compound 23

${ }^{13} \mathrm{C}$ NMR $\left(101 \mathrm{MHz}, \mathrm{CDCl}_{3}\right)$
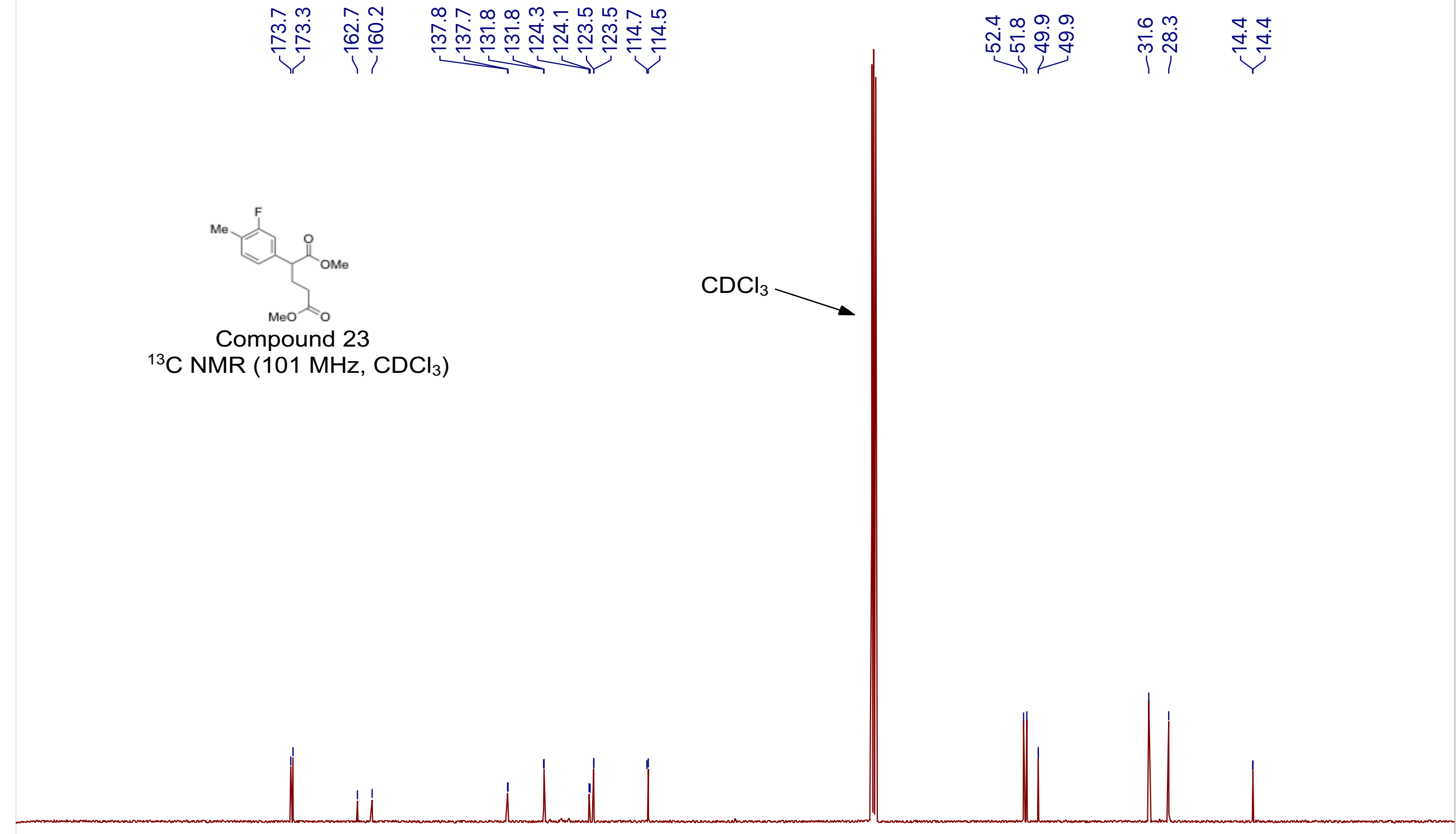


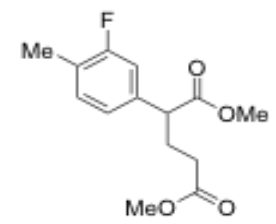

Compound 23

${ }^{19} \mathrm{~F}$ NMR (376 MHz, $\mathrm{CDCl}_{3}$ ) 
ம

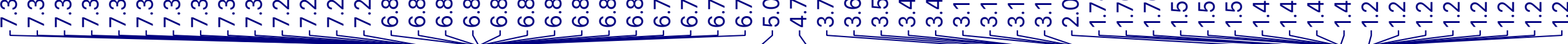

$\mathrm{OMe}$

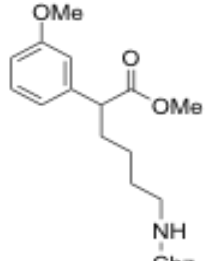

$\mathrm{Cbz}$

Compound 24

${ }^{1} \mathrm{H}$ NMR $\left(400 \mathrm{MHz}, \mathrm{CDCl}_{3}\right)$
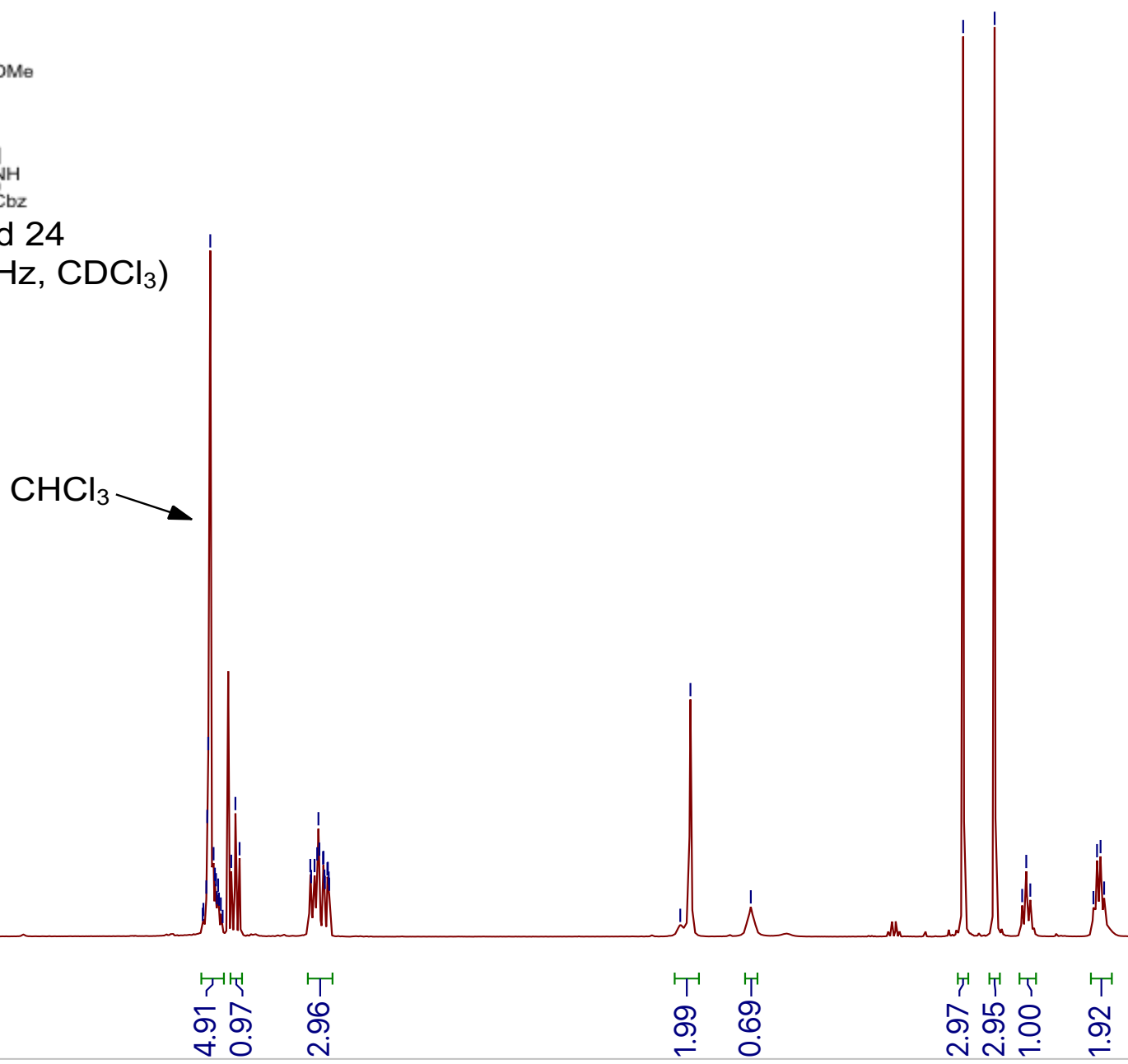

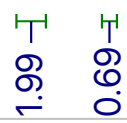

교용

ำ

다난

들율 


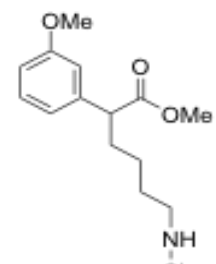

Compound 24

${ }^{13} \mathrm{C}$ NMR (101 MHz, $\mathrm{CDCl}_{3}$ )

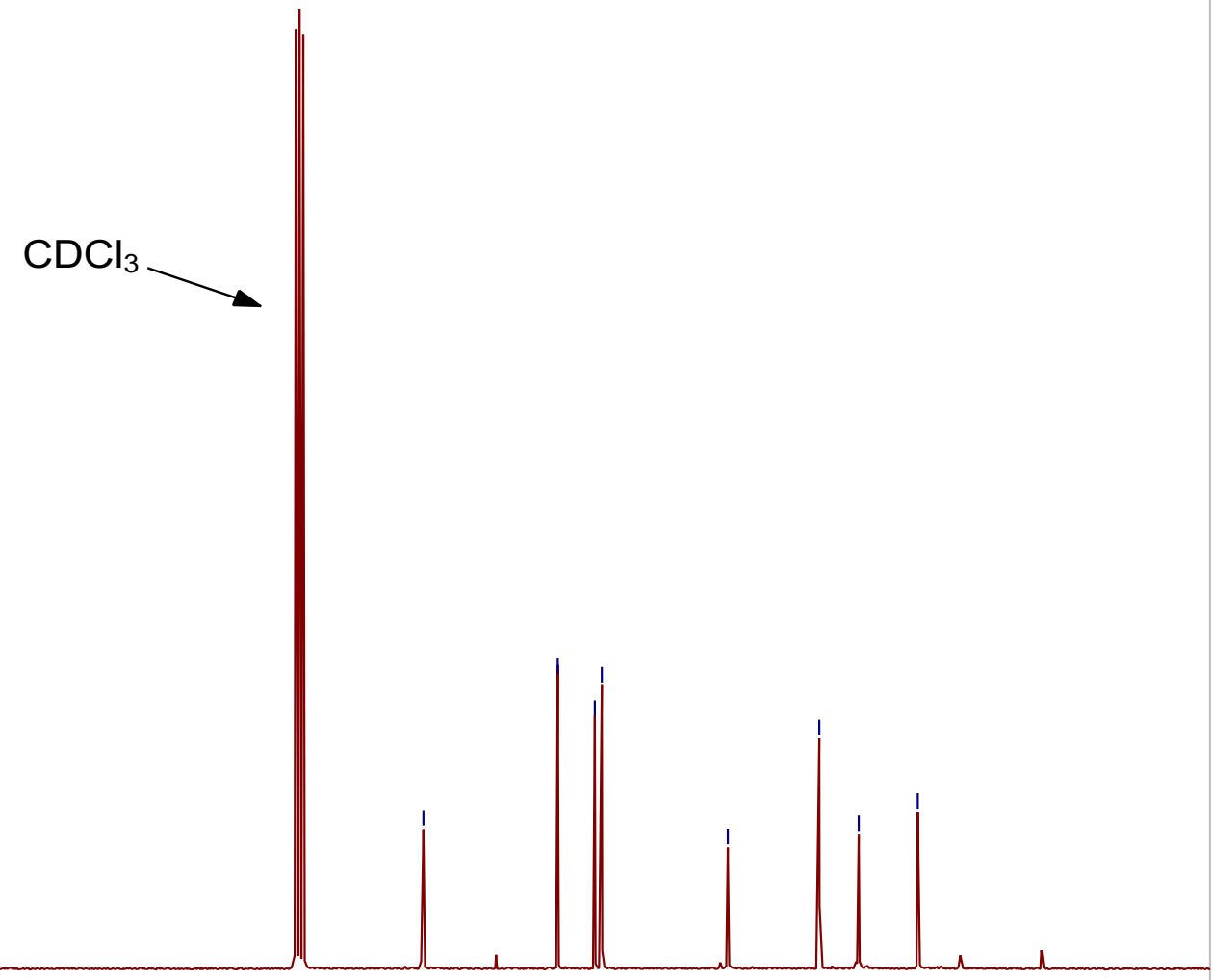

$-2000$

$-1800$

$-1600$

$-1400$

$-1200$

$-1000$

$-800$

$-600$

$-400$

$-200$

$-0$ 

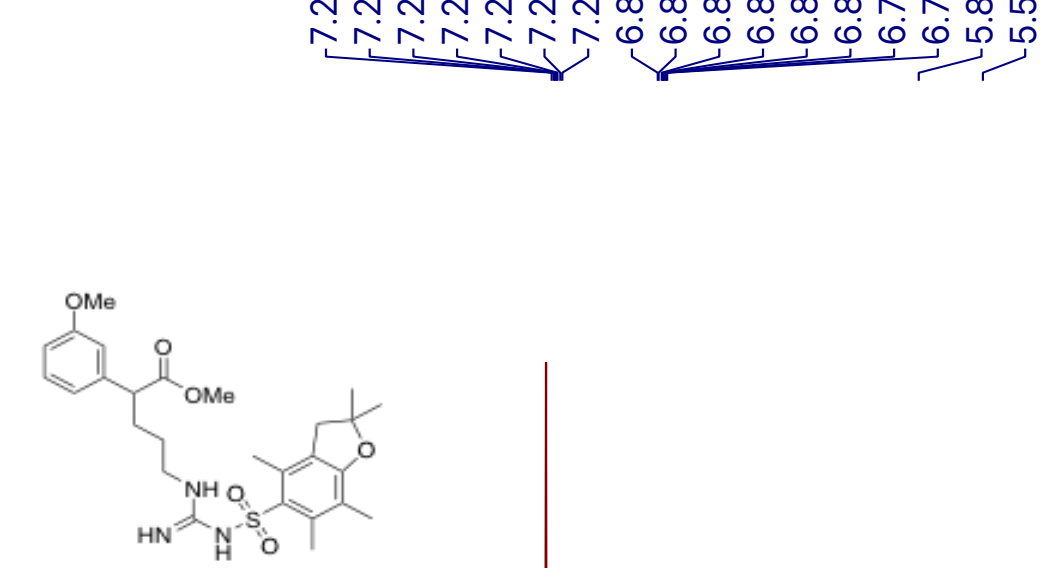

Compound 25

${ }^{1} \mathrm{H}$ NMR (400 MHz, $\mathrm{CDCl}_{3}$ )
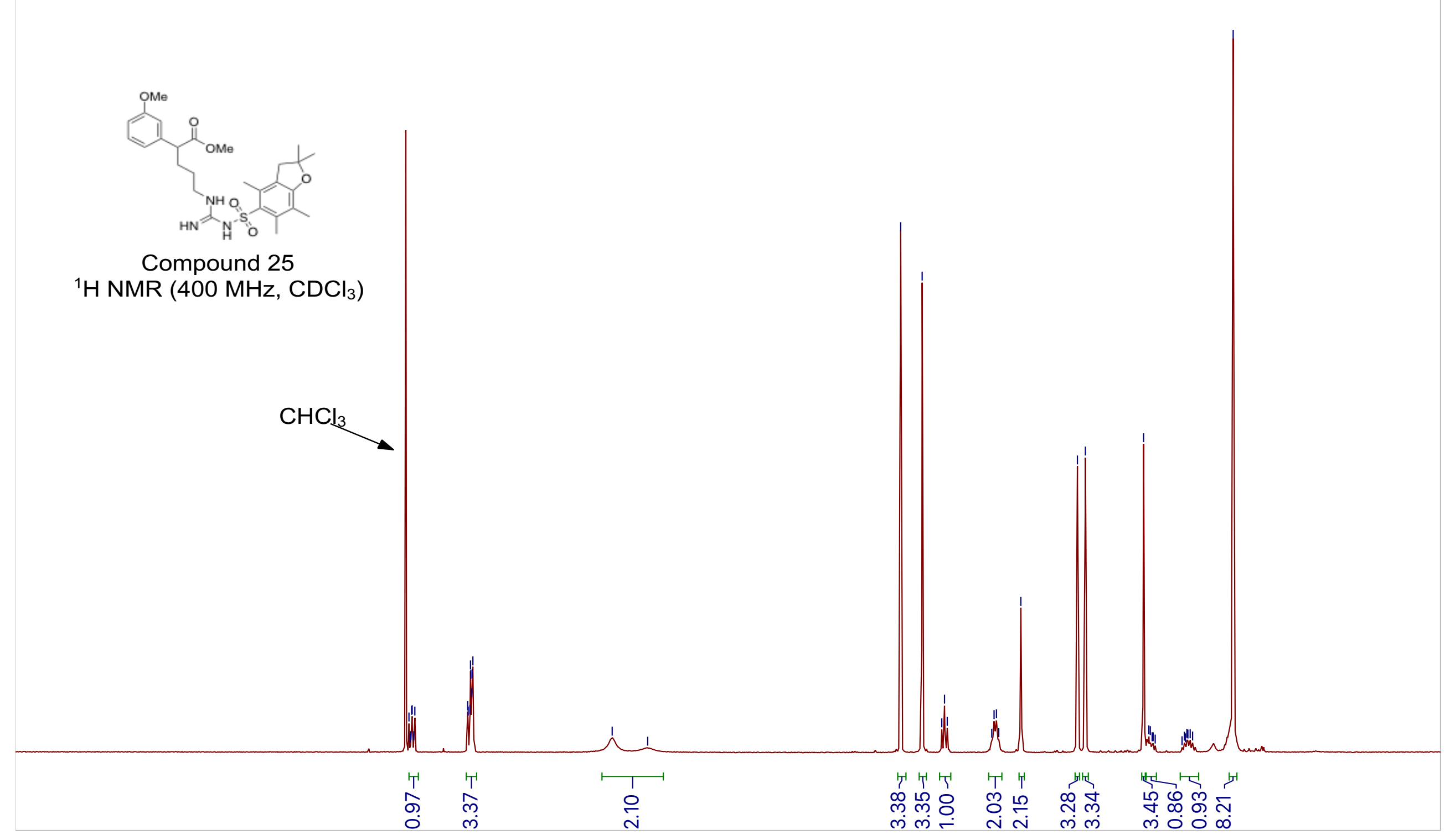


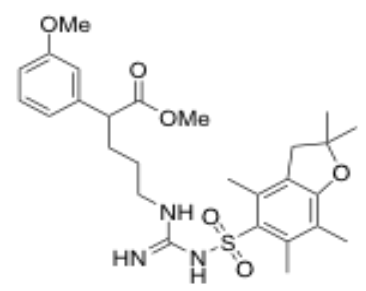

Compound 25

${ }^{13} \mathrm{C}$ NMR $\left(101 \mathrm{MHz}, \mathrm{CDCl}_{3}\right)$
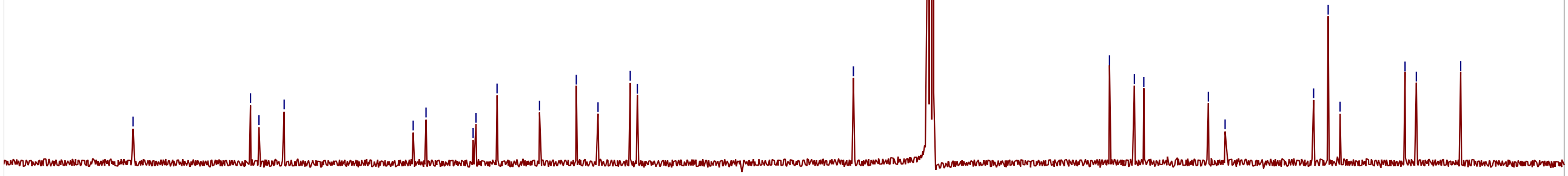
(N)

$\stackrel{\ominus}{B F}_{4} \mathrm{Ph}$

Compound $3 c$

${ }^{1} \mathrm{H}$ NMR (400 MHz, $\mathrm{CDCl}_{3}$ )
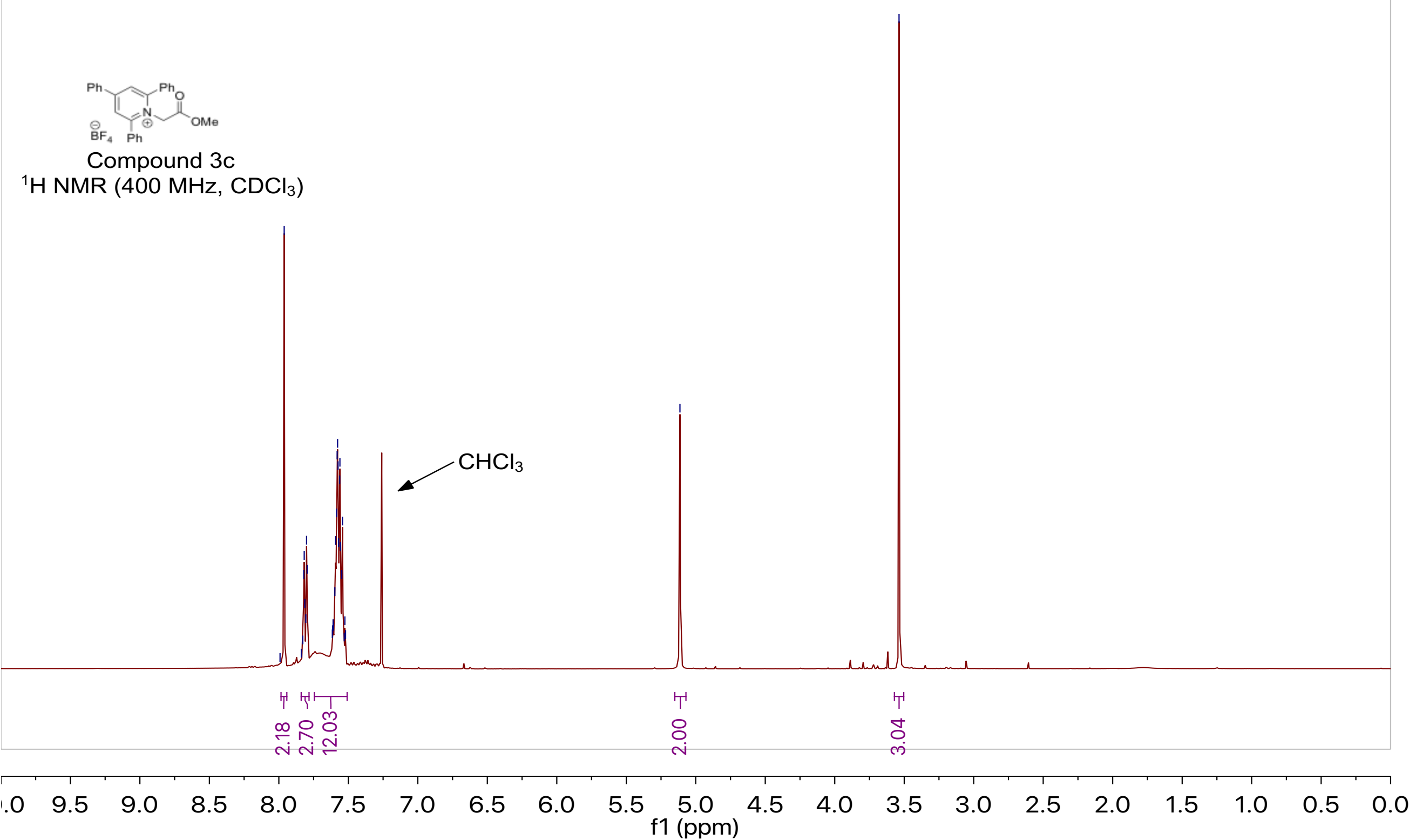

$-2000$ 


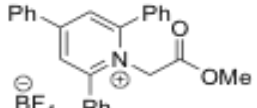

Compound 3c

${ }^{19} \mathrm{~F}$ NMR (565 MHz, $\mathrm{CDCl}_{3}$ )

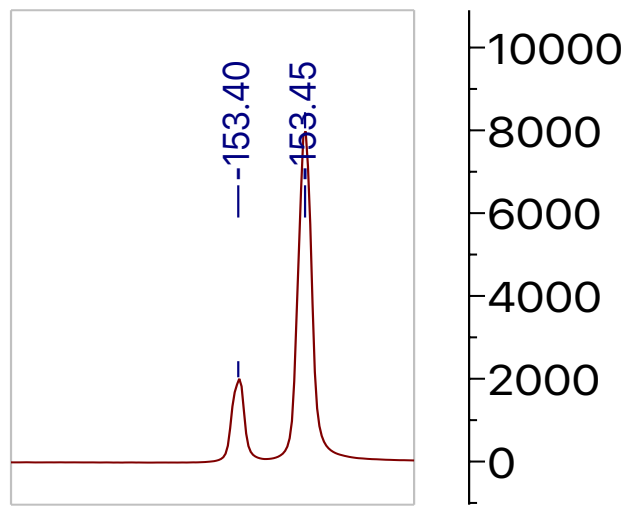

$-10000$

$-9000$

$-8000$

$-7000$

$-6000$

$-5000$

$-4000$

$-3000$

$-153.3 \quad-153.5$

f1 (ppm)

$-2000$

$-1000$

10

$-10$

$-30$

$-50$

$-70$

$-90$

$-110$

$-130$

$-150$

$-170$

$-190$

$-210$ 
${ }^{1} \mathrm{H}$ NMR (400 MHz, $\mathrm{CDCl}_{3}$ )

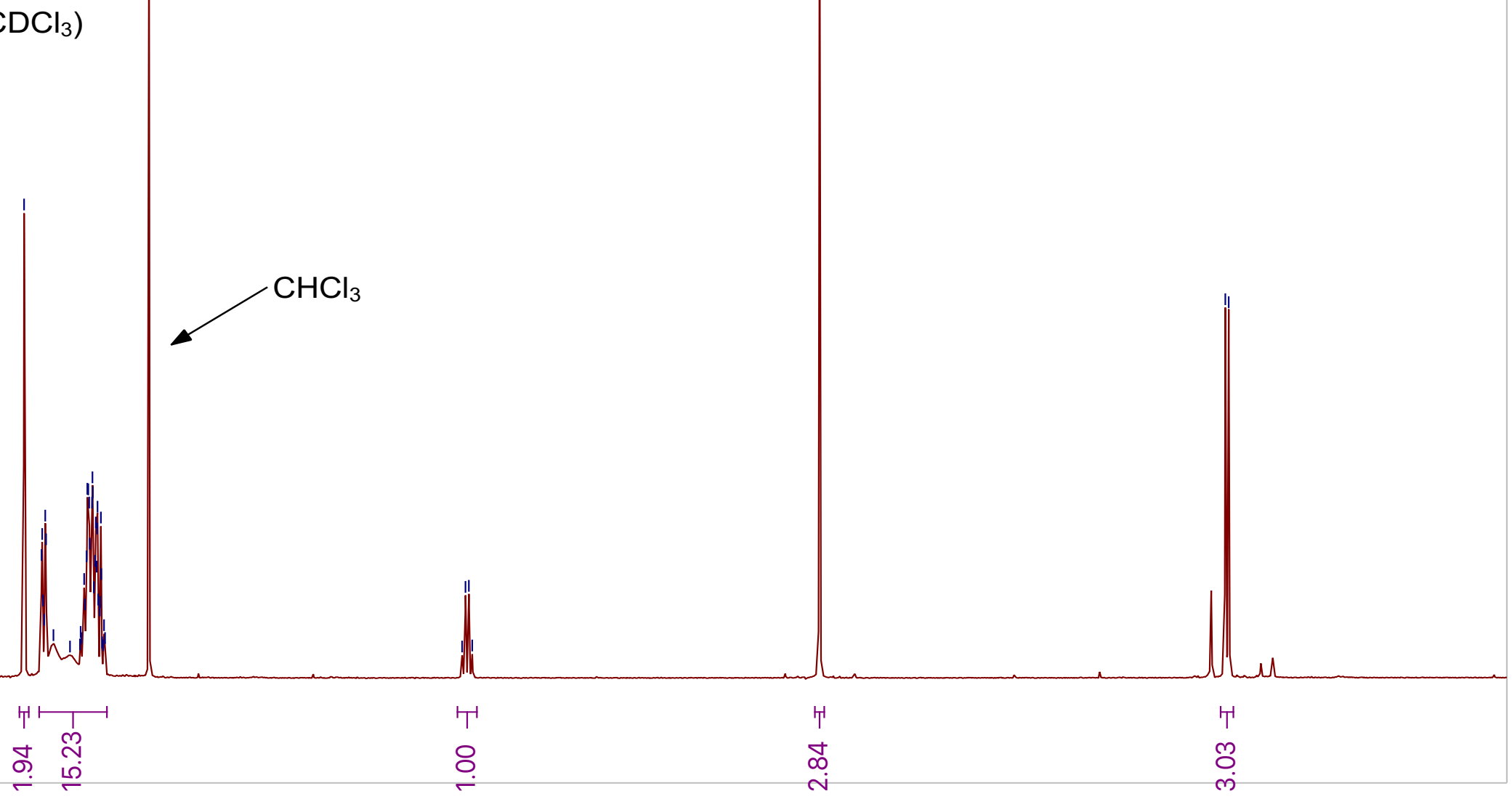


$\mathrm{Ph}$

$\stackrel{\ominus}{\mathrm{BF}_{4}}$

Compound $3 a$

${ }^{13} \mathrm{C}$ NMR $\left(101 \mathrm{MHz}, \mathrm{CDCl}_{3}\right)$

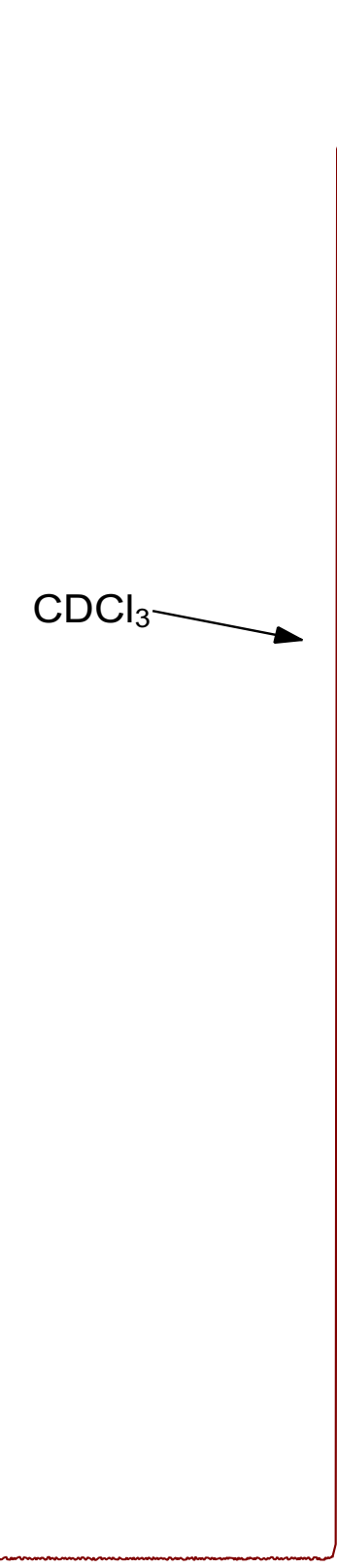




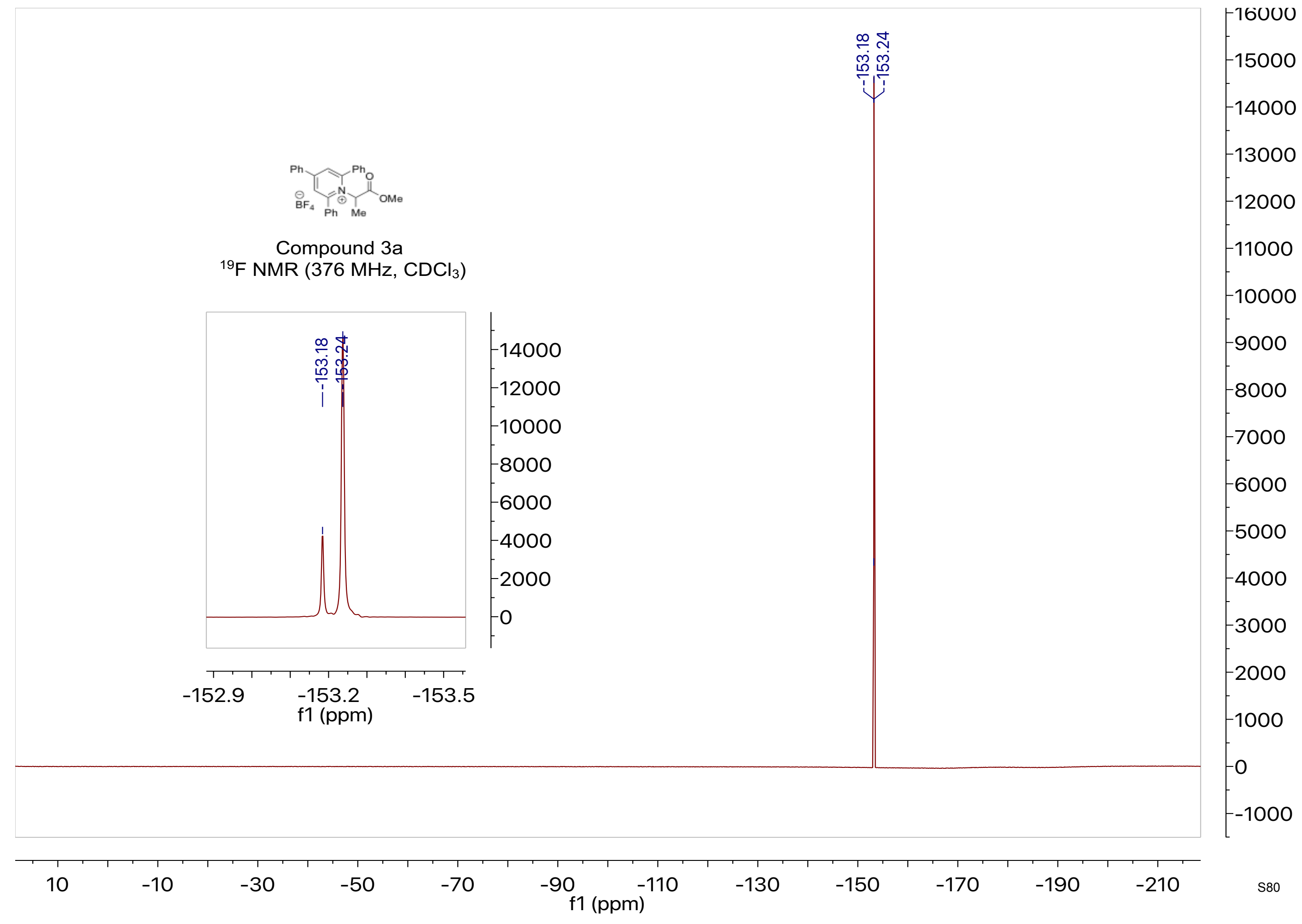



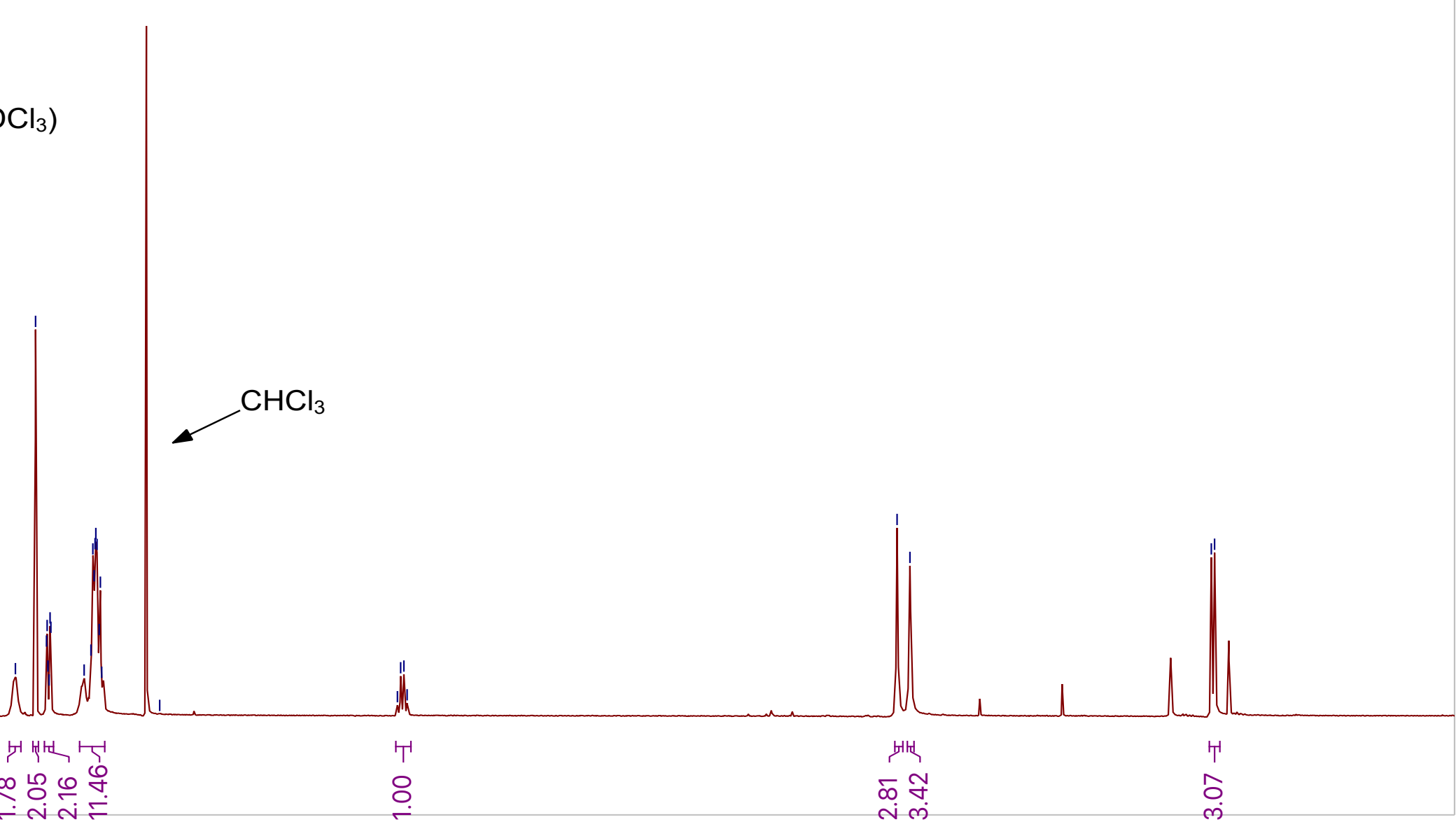


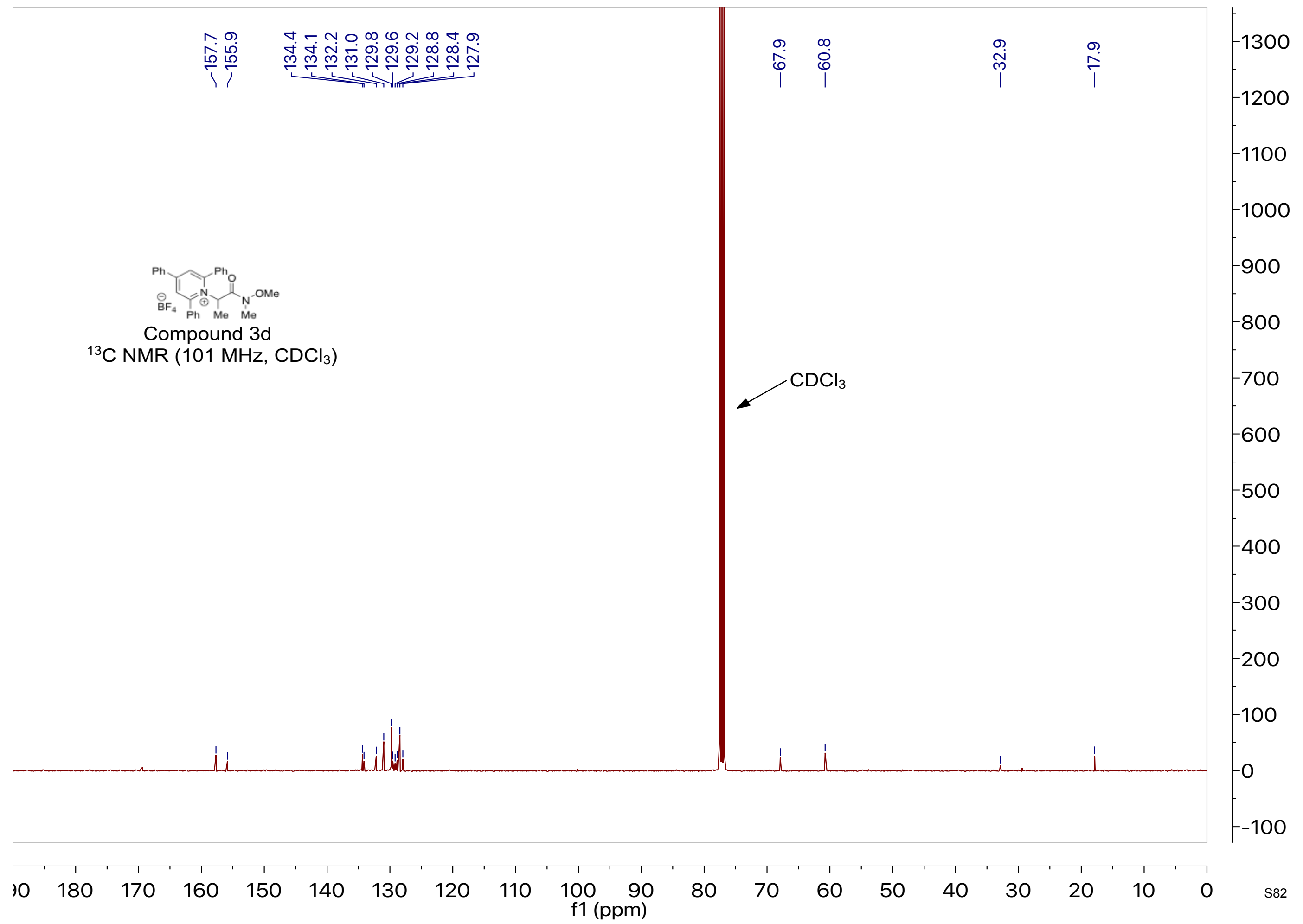




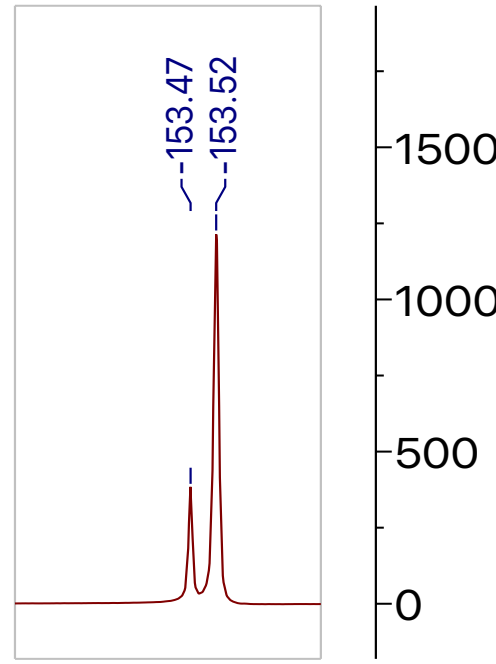

$-1200$

$-1100$

$-1000$

$-900$

$-800$

$-700$

$-600$

$-500$

$-400$

$-153.2-153.6$

$-300$

f1 (ppm) 

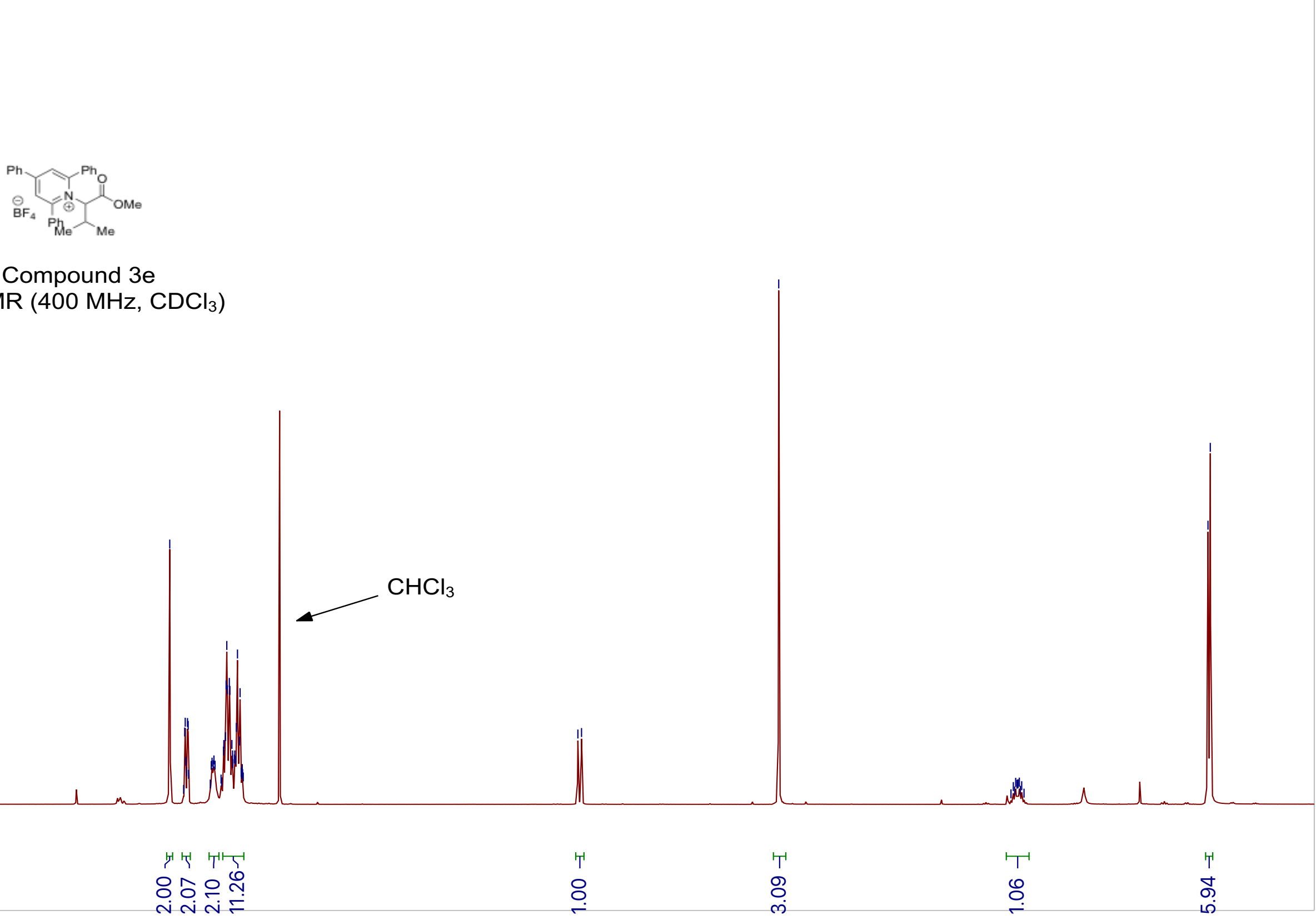

Compound $3 e$

${ }^{1} \mathrm{H}$ NMR $\left(400 \mathrm{MHz}, \mathrm{CDCl}_{3}\right)$

-1200
-1100
-1000
-900
-800
-700
-600
-500
-400
-300
-200
-100
-0
-100
-200

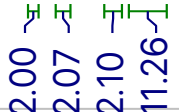

$\mathrm{CHCl}_{3}$

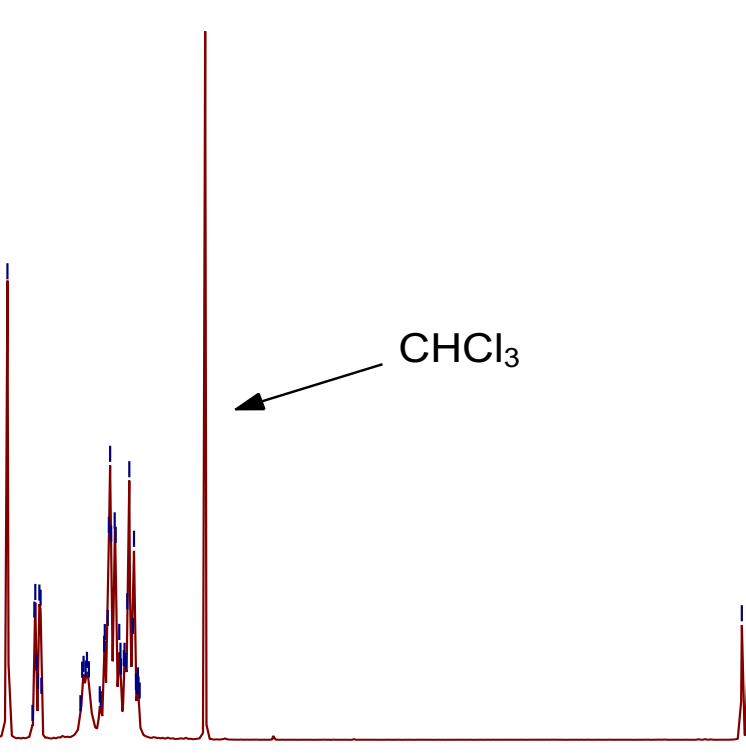

4.0 

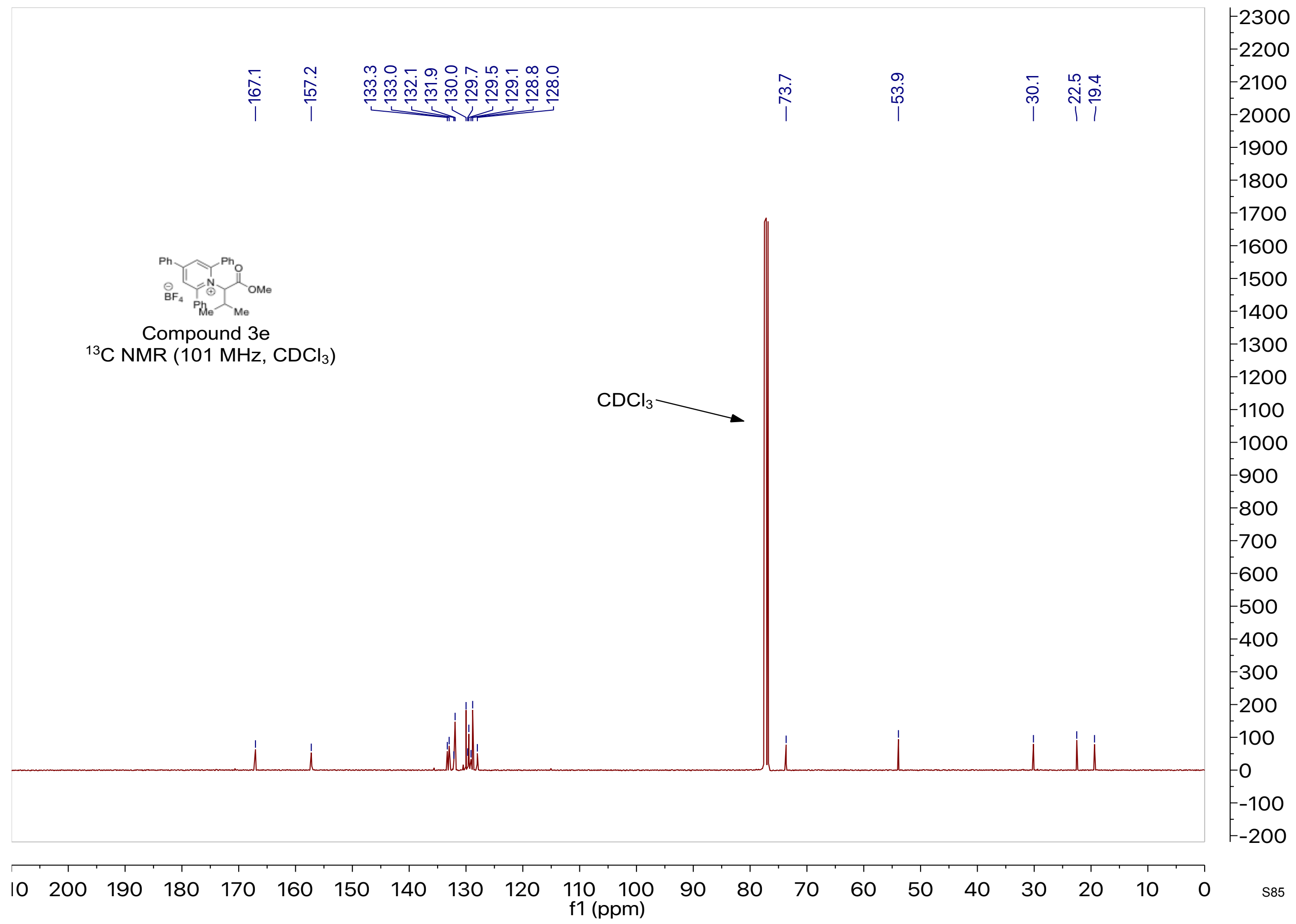

S85 


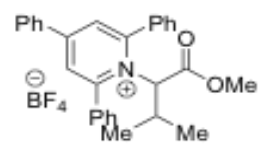

Compound $3 e$

${ }^{19} \mathrm{~F}$ NMR (376 MHz, $\mathrm{CDCl}_{3}$ )

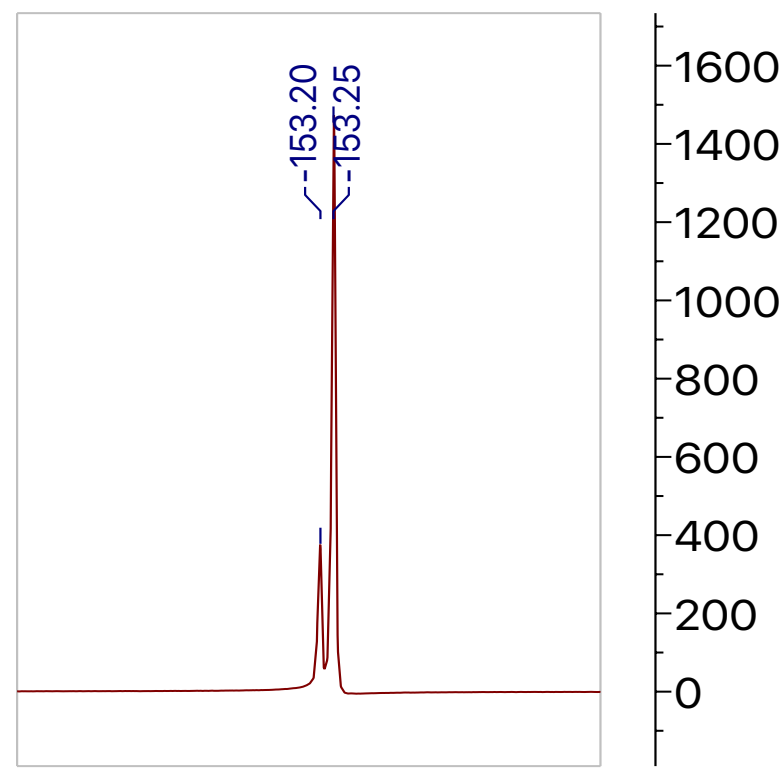

$-153.0$

$-154.0$

f1 (ppm) 


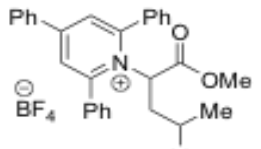

Me

Compound $3 f$

${ }^{1} \mathrm{H}$ NMR $\left(600 \mathrm{MHz}, \mathrm{CDCl}_{3}\right)$

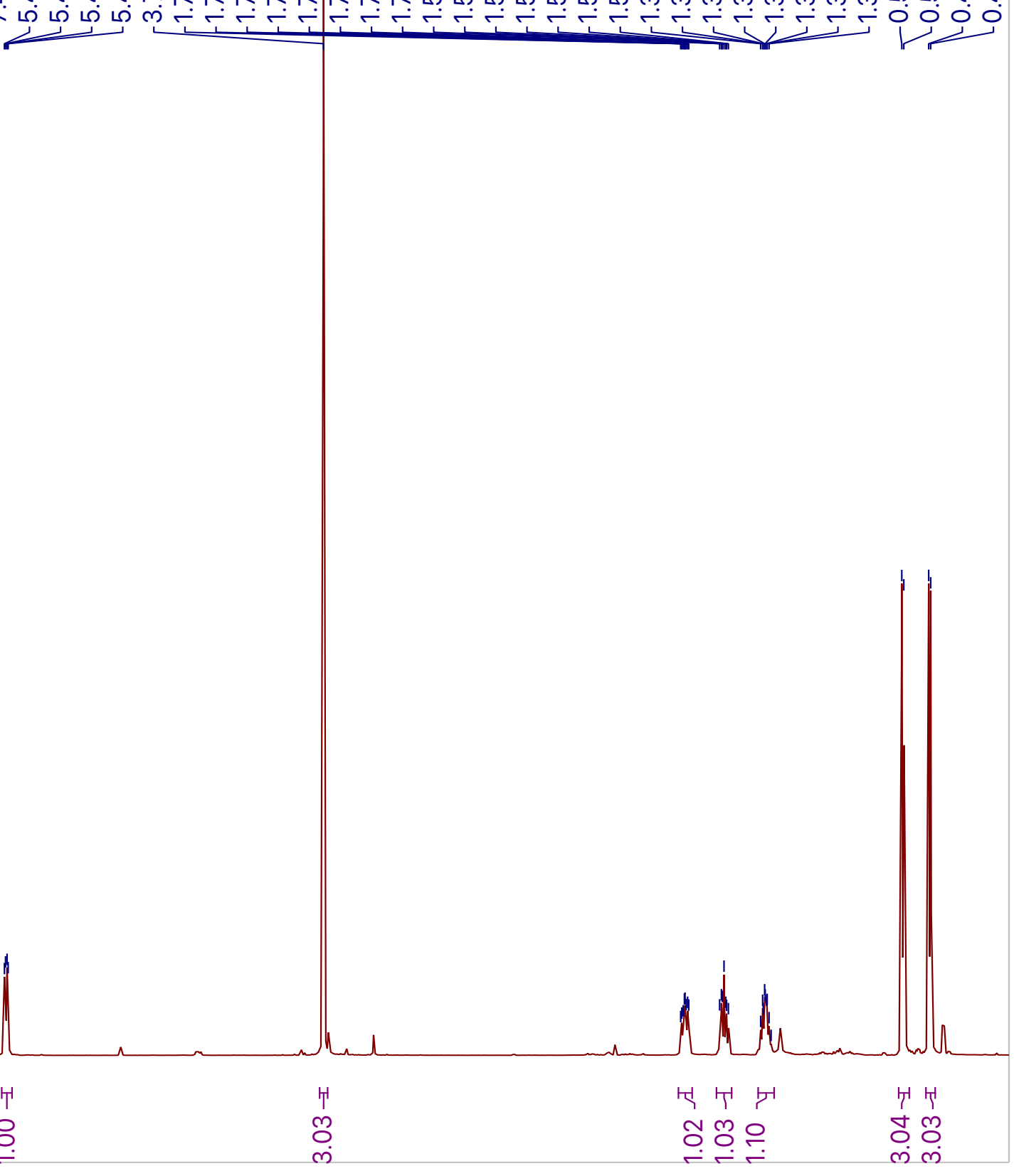

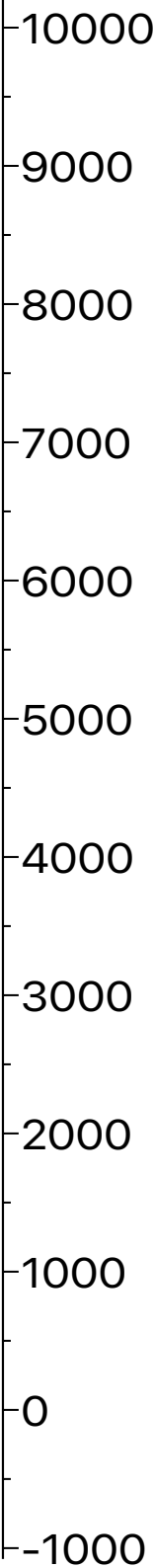

$\infty \sim m$

ìi

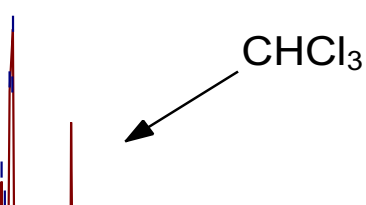

8.08 .0

7.57 .0

6.5

6.0

$5.5 \quad 5.0$ f1 (ppm) 


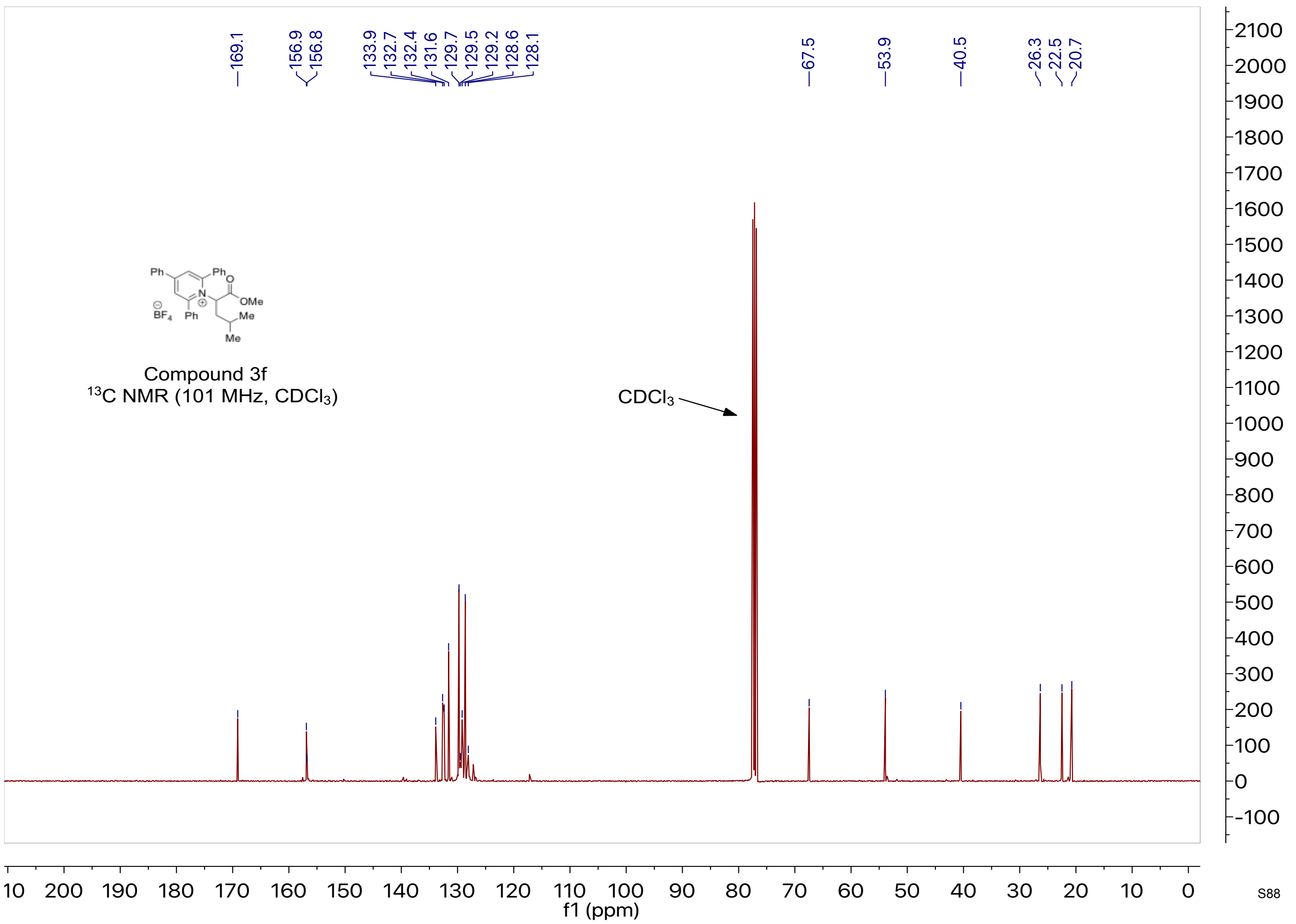




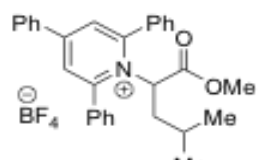

Compound $3 f$ ${ }^{19} \mathrm{~F}$ NMR (376 MHz, $\mathrm{CDCl}_{3}$ )

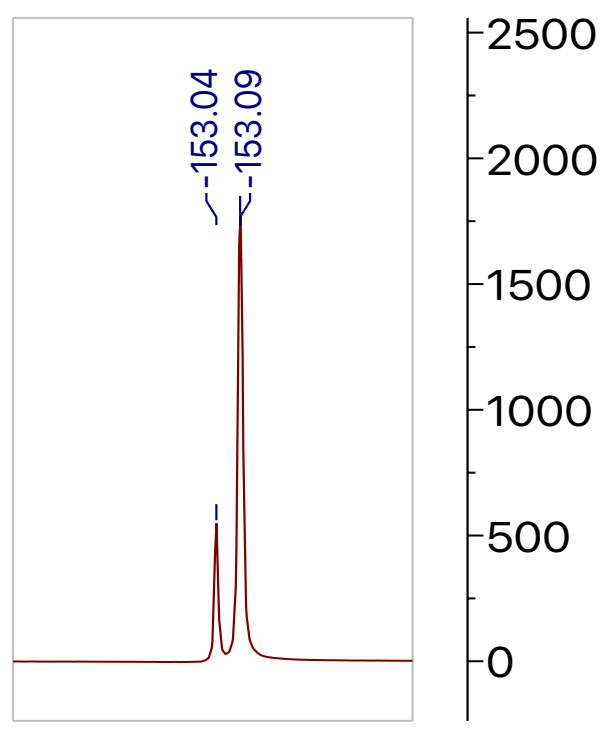



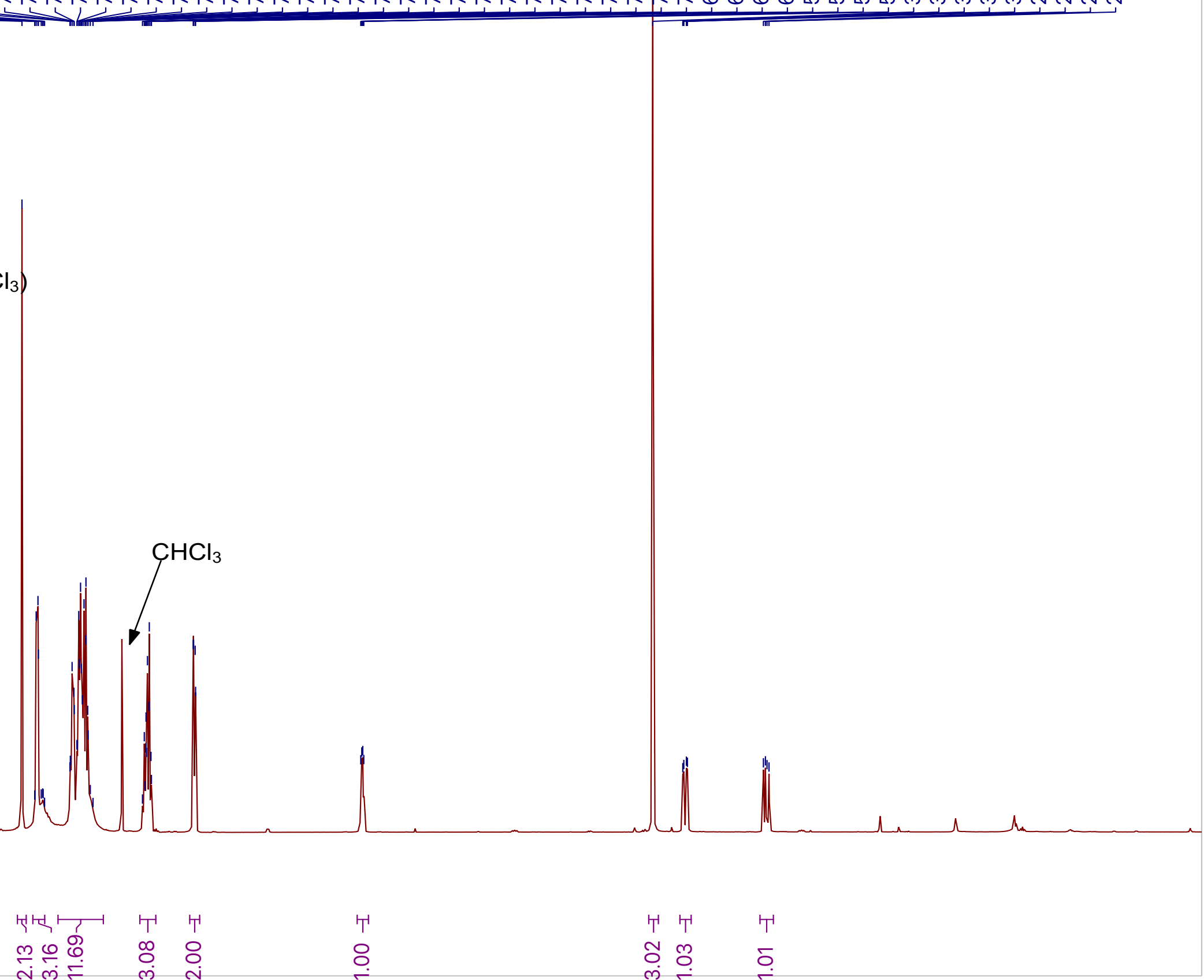

6500

$-6000$

$-5500$

$-5000$

$-4500$

$-4000$

$-3500$

$-3000$

$-2500$

$-2000$

$-1500$

$-1000$

$-500$

- 


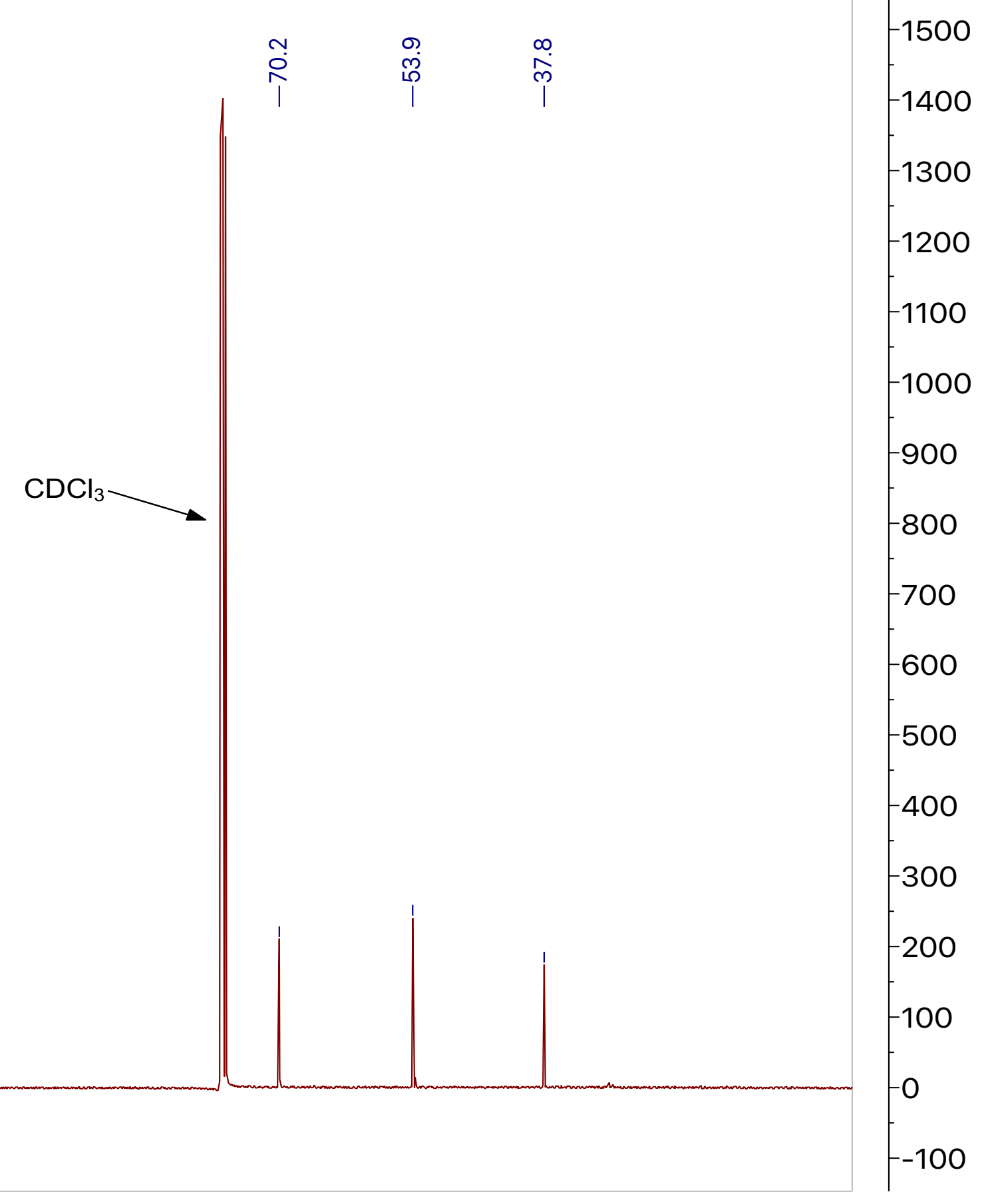

Compound $3 \mathrm{~g}$

${ }^{13} \mathrm{C}$ NMR $\left(101 \mathrm{MHz}, \mathrm{CDCl}_{3}\right)$

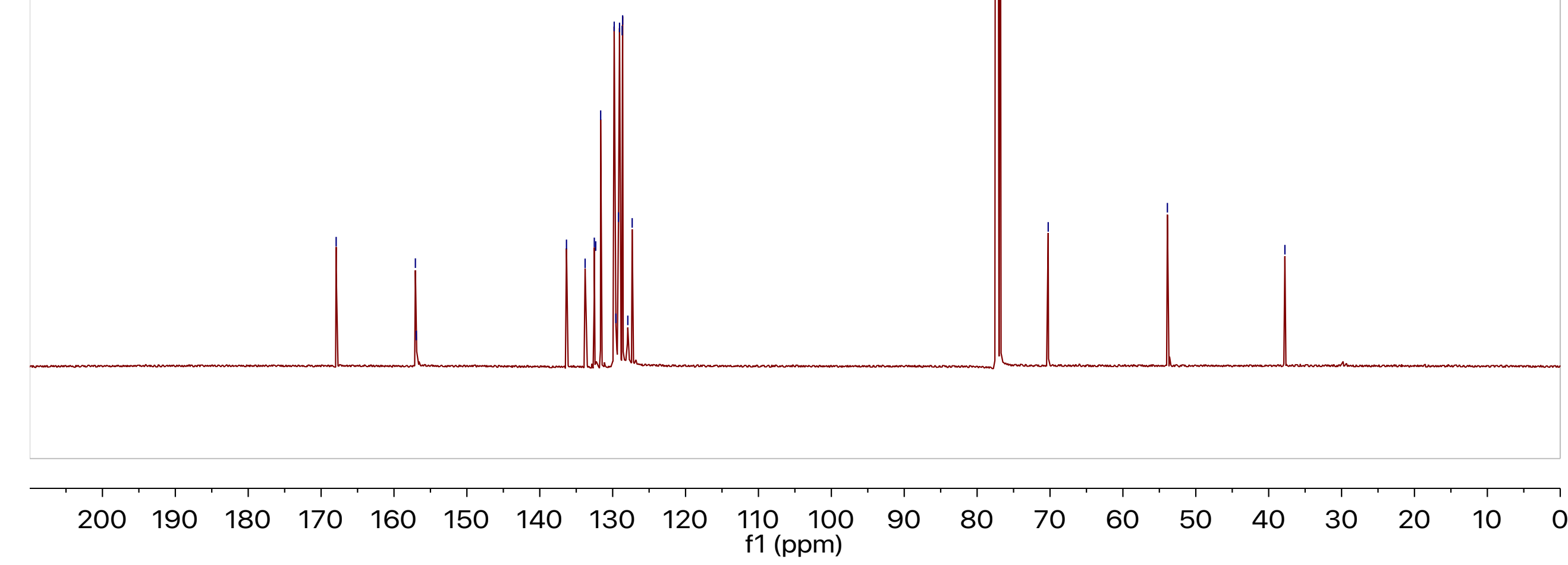


${ }^{19} \mathrm{~F}$ NMR (376 MHz, $\mathrm{CDCl}_{3}$ )

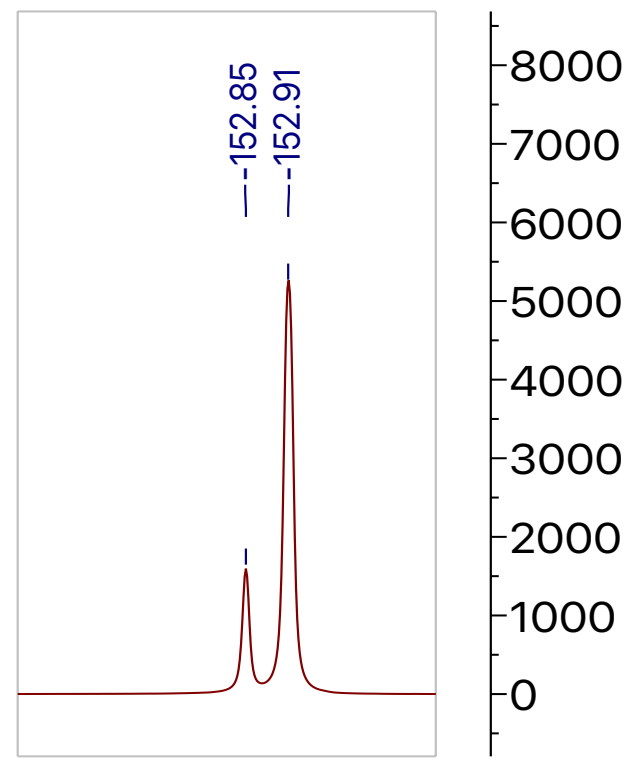

$-5500$

$-5000$

$-4500$

$-4000$

$-3500$

$-3000$

$-2500$

$-2000$

$-1500$

$-152.7 \quad-153.0$

f1 (ppm)

$-1000$

$-500$

$-0$ 


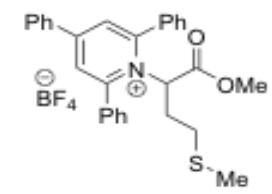

Compound $3 \mathrm{~h}$

${ }^{1} \mathrm{H}$ NMR (400 MHz, $\mathrm{CDCl}_{3}$ )

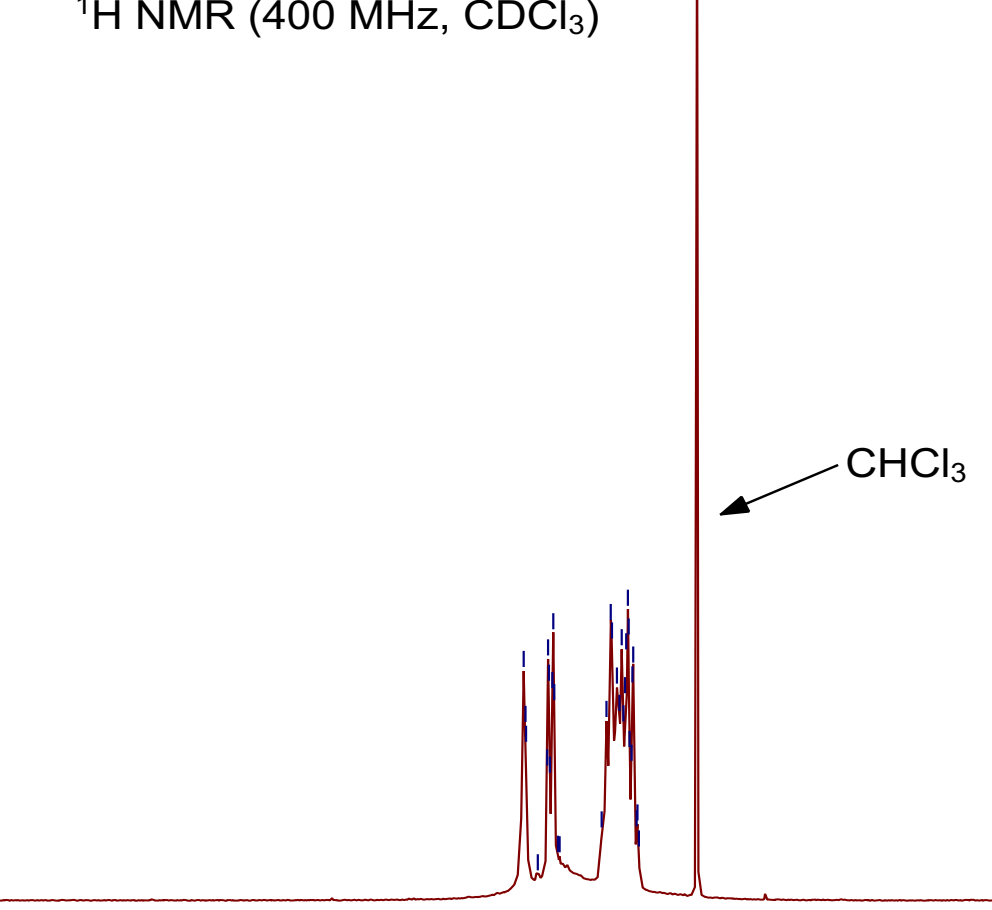

Tा th

ต ต

m
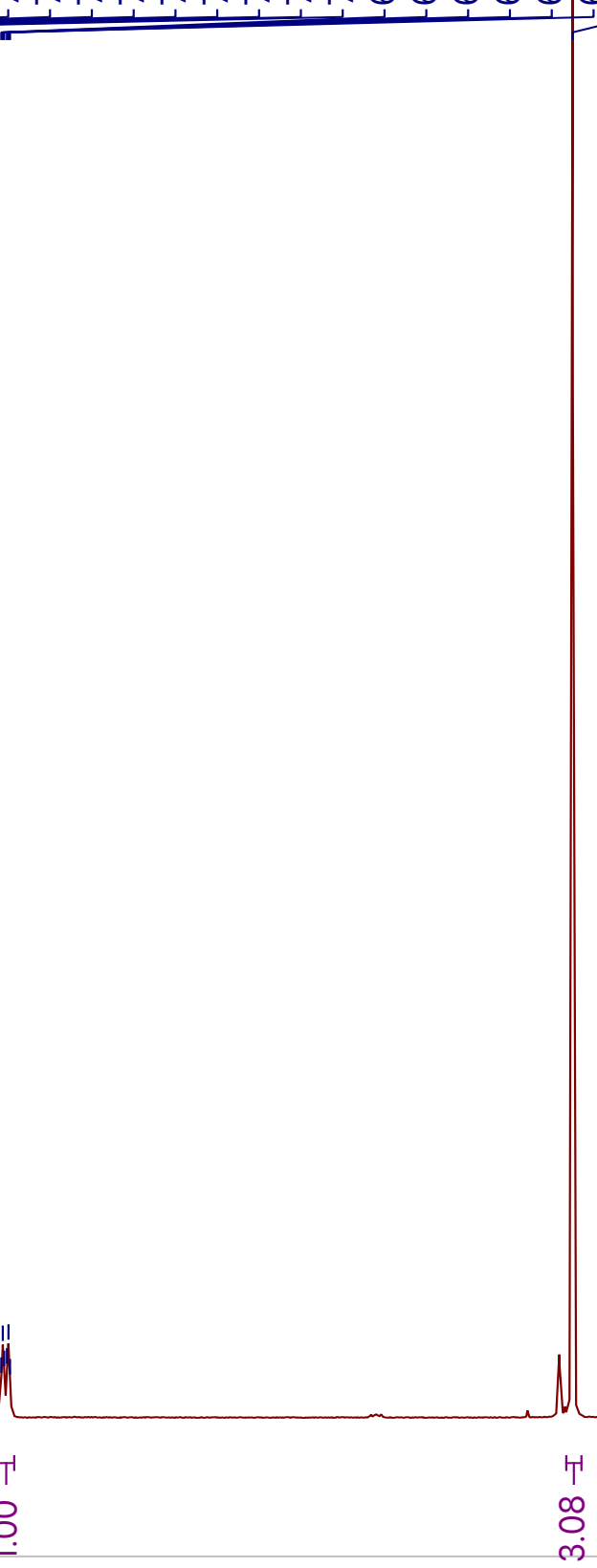
S

Compound $3 \mathrm{~h}$

${ }^{13} \mathrm{C}$ NMR $\left(101 \mathrm{MHz}, \mathrm{CDCl}_{3}\right)$

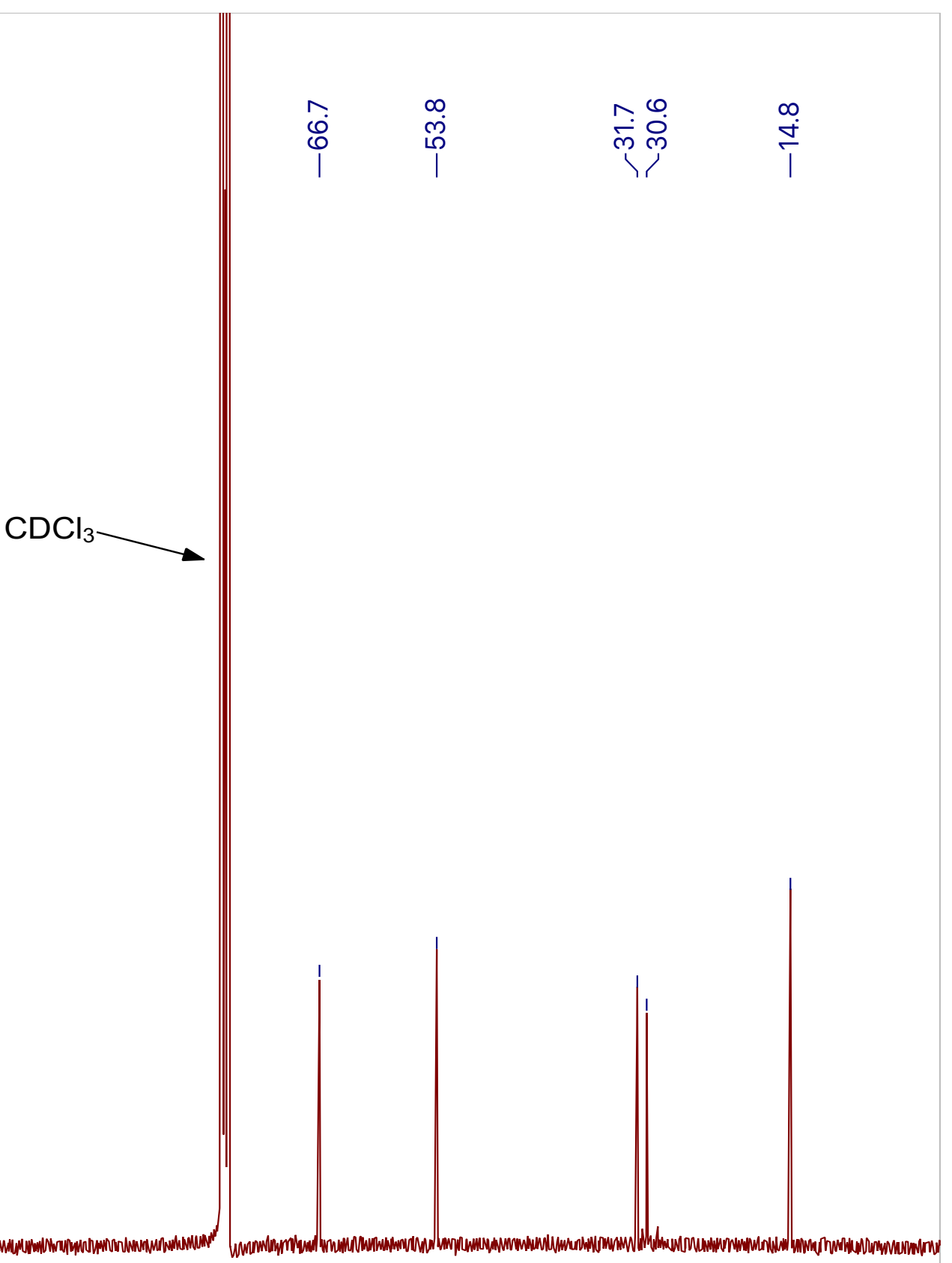

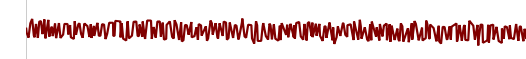

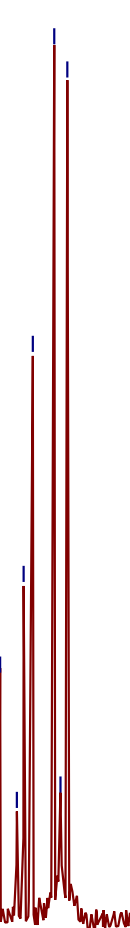

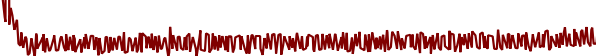




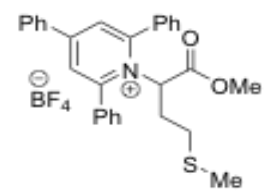

Compound $3 \mathrm{~h}$

${ }^{19} \mathrm{~F}$ NMR (376 MHz, $\mathrm{CDCl}_{3}$ )

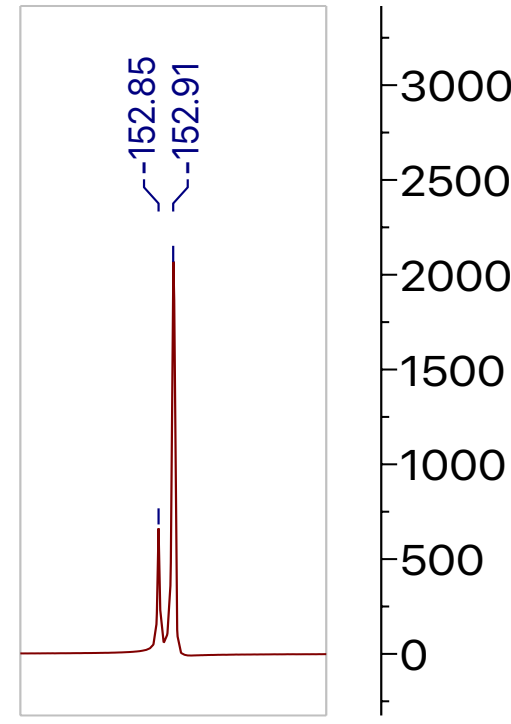

$-2000$ $-1800$ $-1600$ $-1400$ $-1200$ $-1000$ $-800$ $-600$

$-152.5-153.0$

f1 (ppm) 
Compound $3 i$

${ }^{1} \mathrm{H}$ NMR $\left(400 \mathrm{MHz}, \mathrm{CDCl}_{3}\right)$

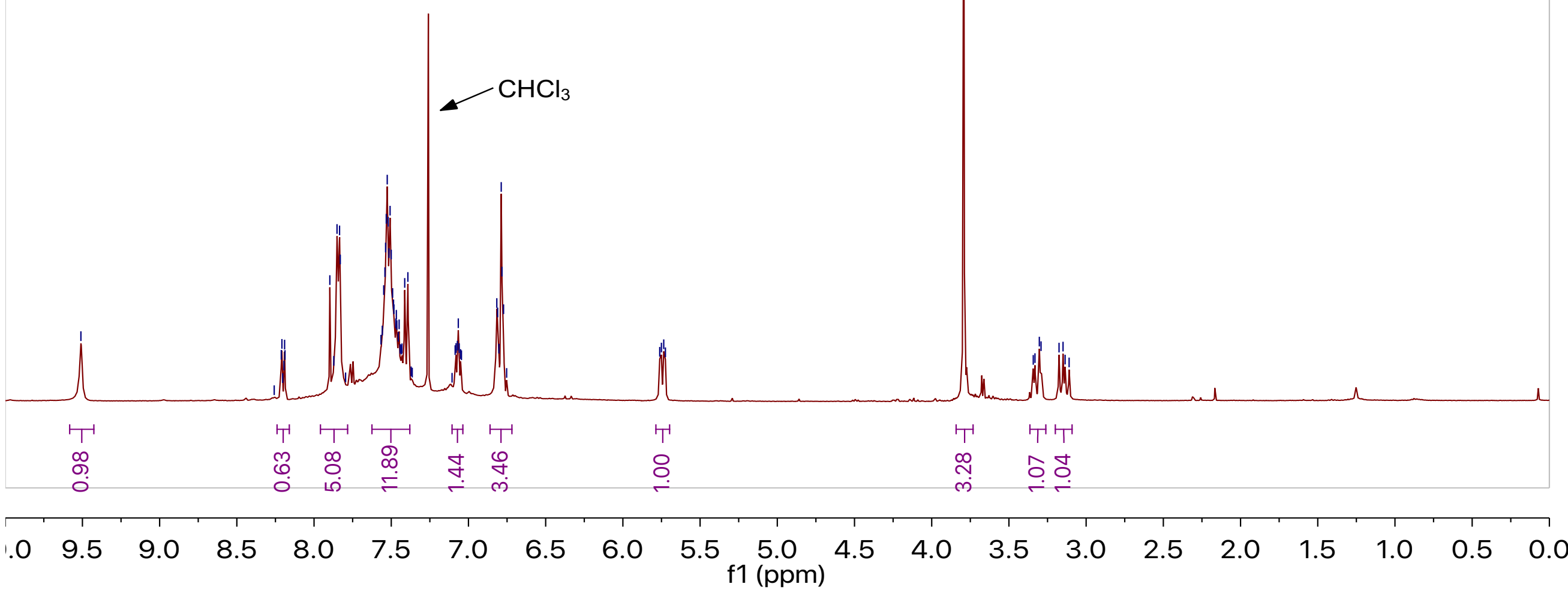




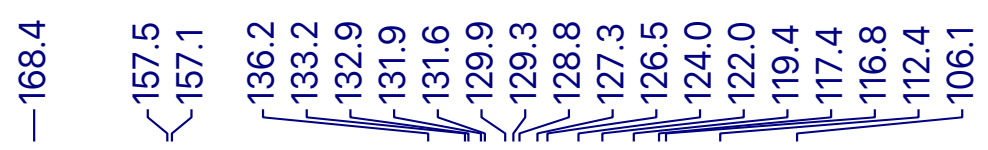

200

190180

$170160 \quad 150$

140

130

120

110100

90

80

70

$\begin{array}{lllllll}60 & 50 & 40 & 30 & 20 & 10 & 0\end{array}$

S97 f1 (ppm) 
Compound 3

${ }^{19} \mathrm{~F} \mathrm{NMR} \mathrm{(376} \mathrm{MHz,} \mathrm{CDCl}_{3}$ )

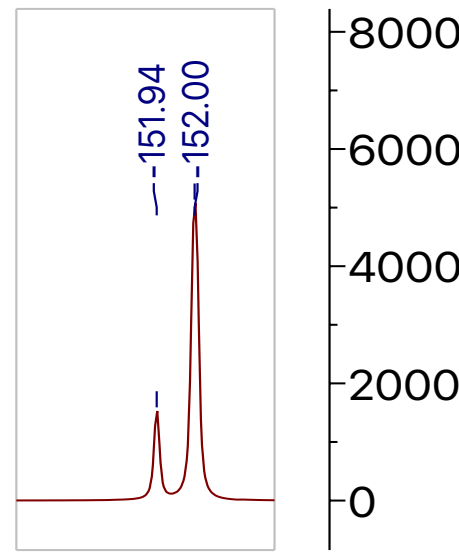

$-4500$

$-4000$

$-3500$

$-3000$

$-2500$

$-2000$

$-1500$

f1 (ppm)

$-152.1$ 


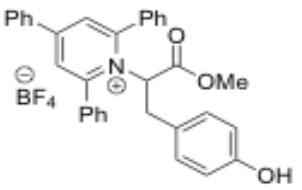

Compound 3

${ }^{1} \mathrm{H} \mathrm{NMR}\left(400 \mathrm{MHz}, \mathrm{CDCl}_{3}\right)$ 


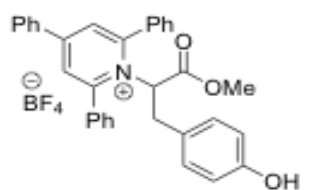

Compound 3j

${ }^{13} \mathrm{C}$ NMR $\left(101 \mathrm{MHz}, \mathrm{CDCl}_{3}\right)$
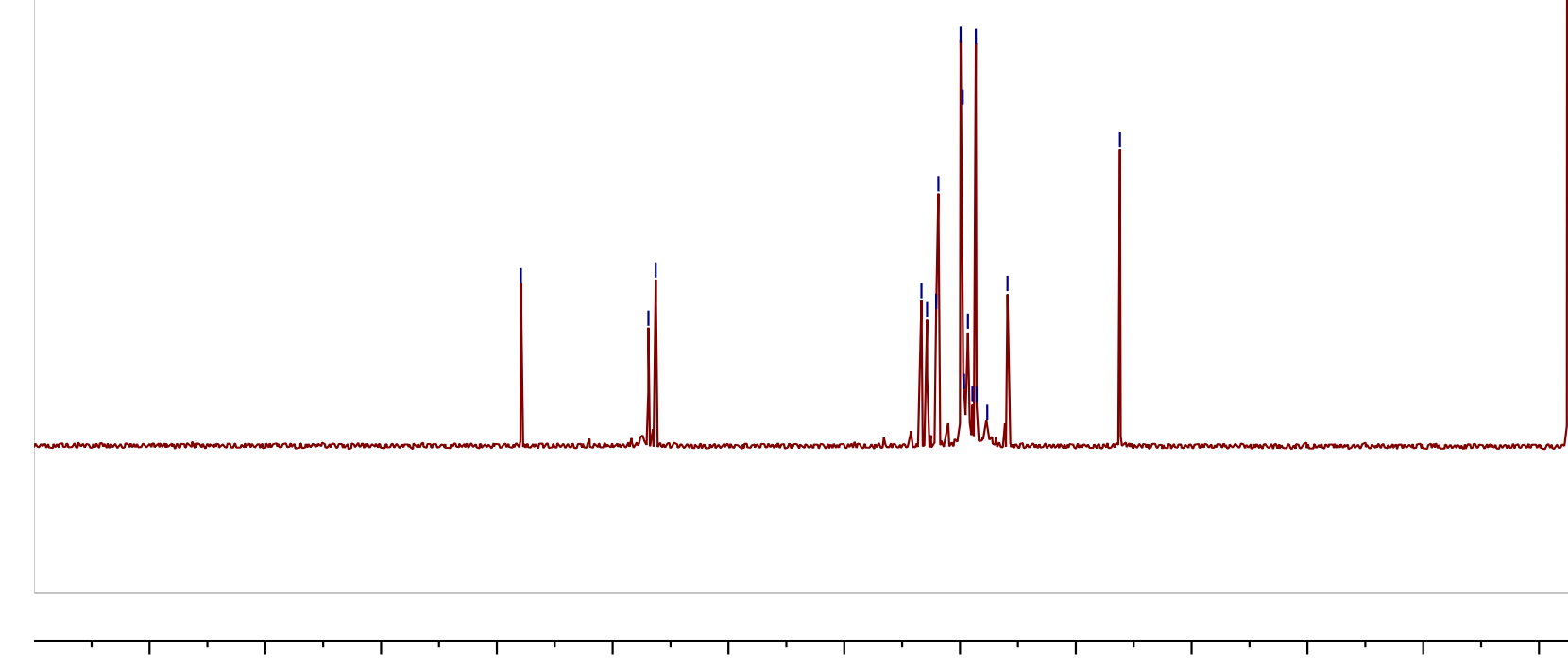


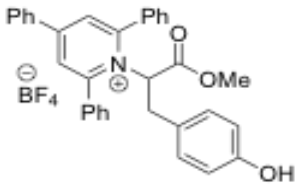

Compound $3 \mathrm{j}$

${ }^{19} \mathrm{~F} \mathrm{NMR}\left(376 \mathrm{MHz}, \mathrm{CDCl}_{3}\right)$

-3000
-2500
-2000
-1500
-500
-0




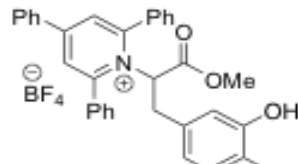

Compound $3 \mathrm{k}$

${ }^{1} \mathrm{H}$ NMR (400 MHz, $\mathrm{CDCl}_{3}$ )
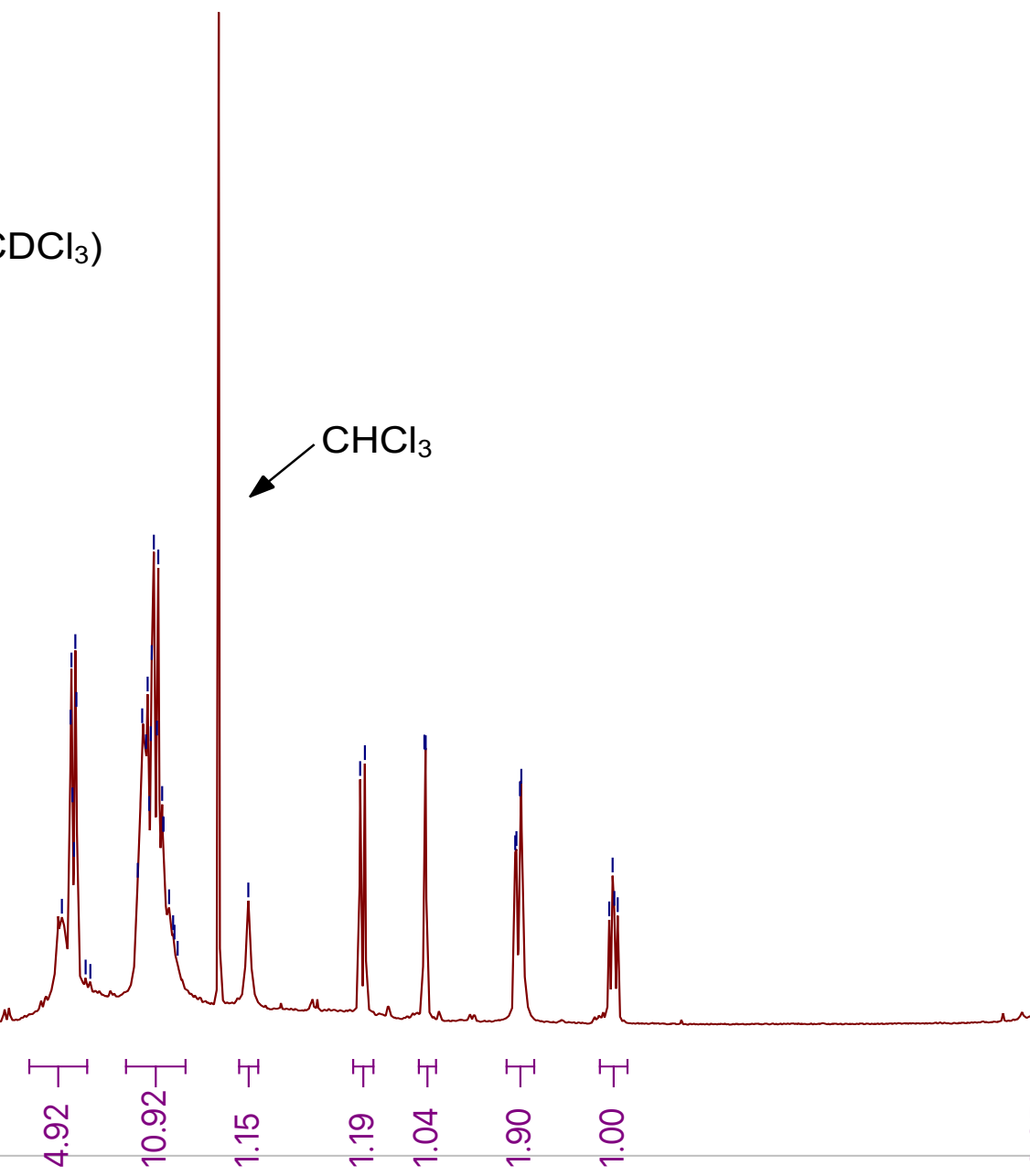
I

$-1200$

Compound $3 \mathrm{~K}$

${ }^{13} \mathrm{C}$ NMR $\left(101 \mathrm{MHz}, \mathrm{CDCl}_{3}\right)$

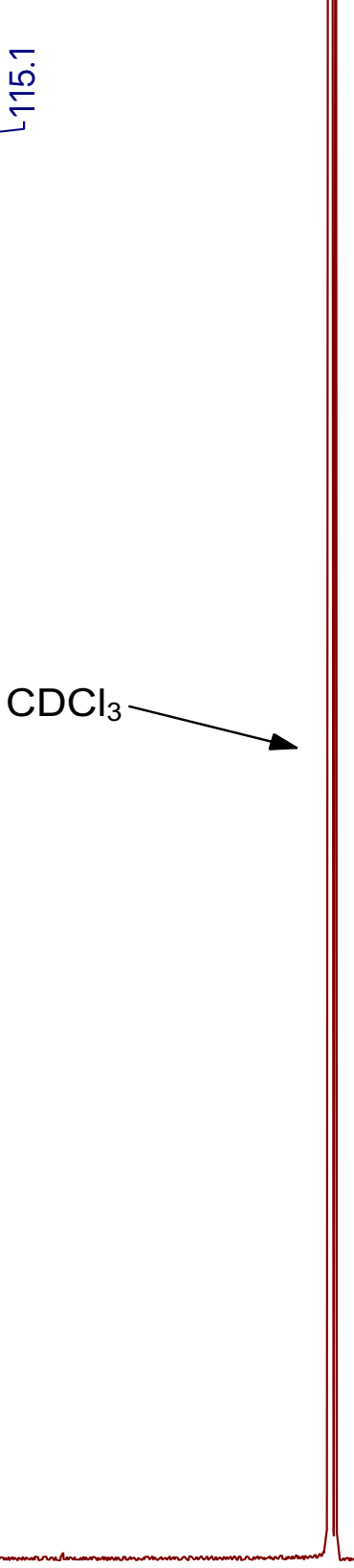

$\stackrel{\circ}{i}$

ז্்

i্

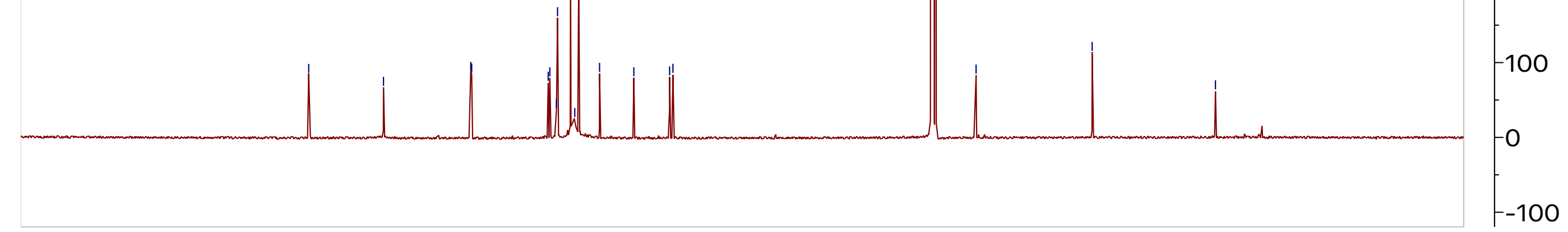


Compound $3 \mathrm{k}$
${ }^{19} \mathrm{~F}$ NMR $\left(376 \mathrm{MHz}, \mathrm{CDCl}_{3}\right)$
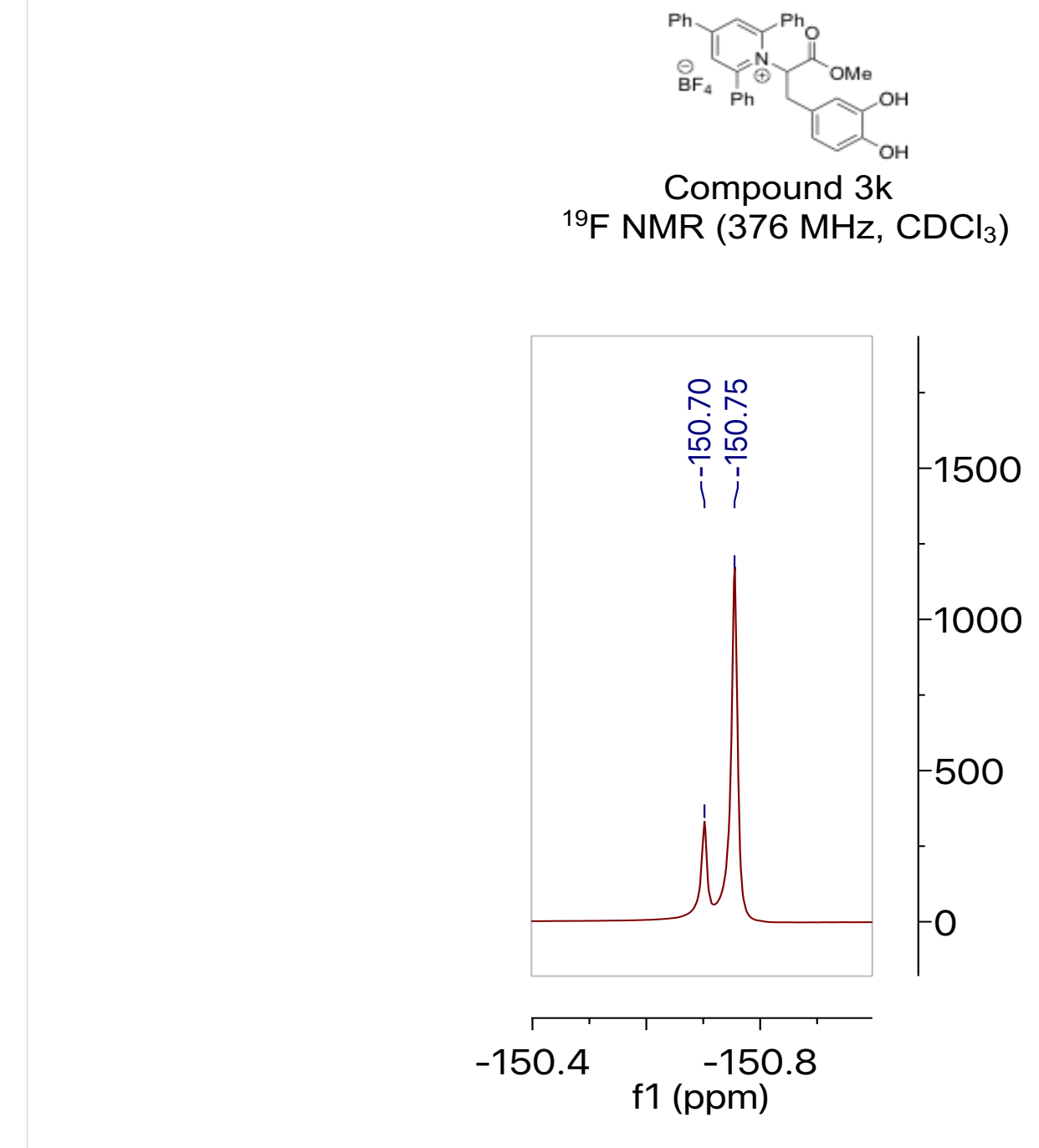
$\mathrm{Ph}^{\mathrm{Ph}} \mathrm{Ph}$

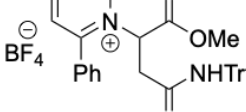

Compound 3

${ }^{1} \mathrm{H}$ NMR $\left(400 \mathrm{MHz}, \mathrm{CDCl}_{3}\right)$
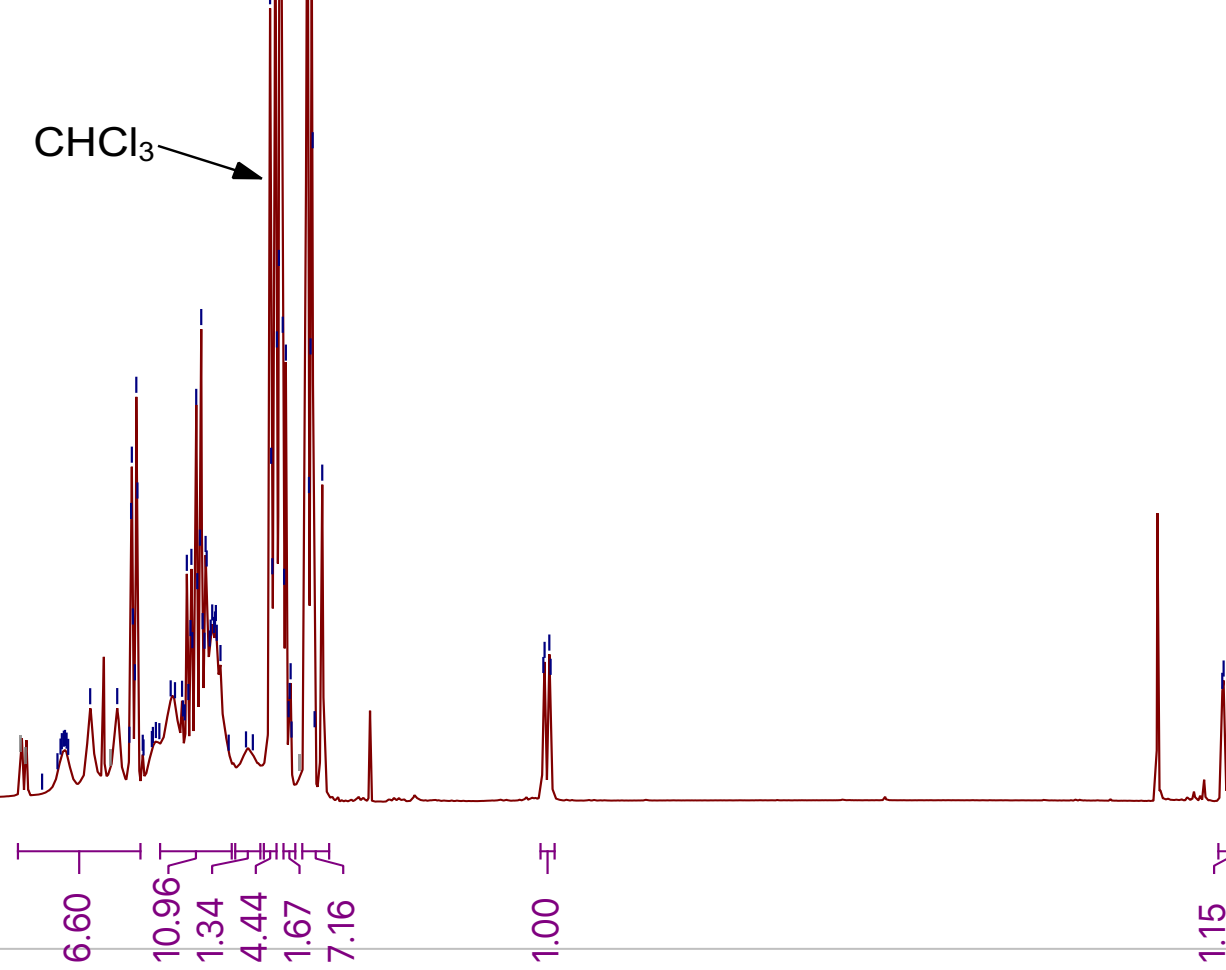

$-1100$

$-1000$

$-900$

$-800$

$-700$

$-600$

$-500$

$-400$

$-300$

$-200$

$-100$

-

$-100$ 


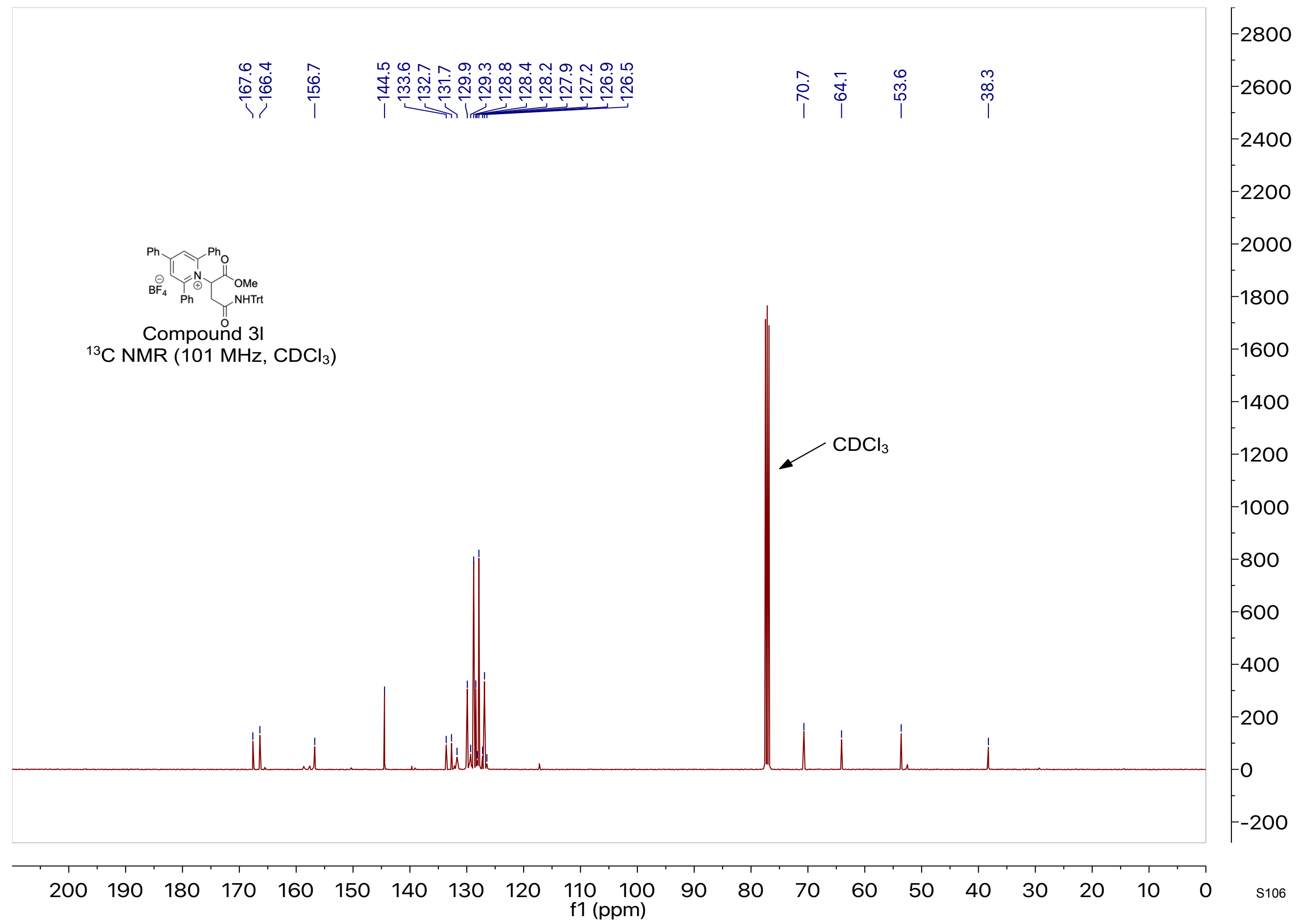




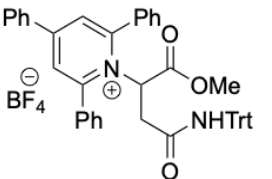

Compound 31

${ }^{19} \mathrm{~F}$ NMR (376 MHz, $\mathrm{CDCl}_{3}$ )

$-6000$

$-5500$

$-5000$

$-4500$

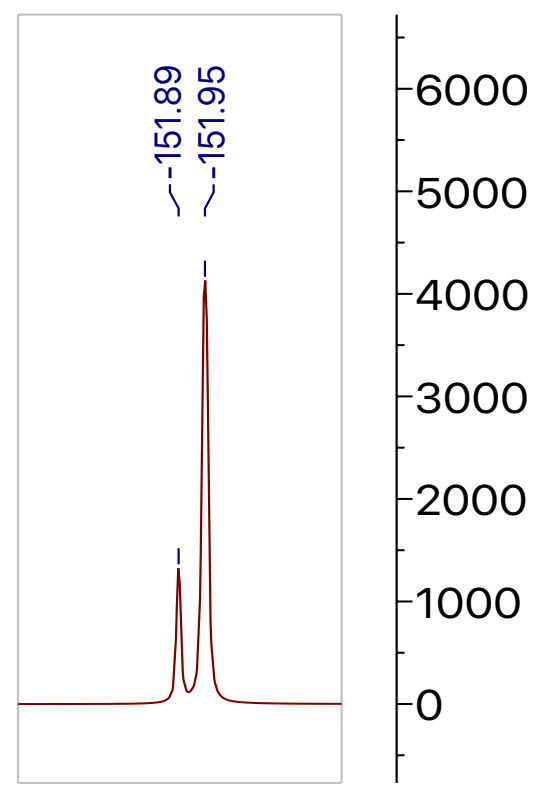

$-4000$

$-3500$

$-3000$

$-2500$

$-2000$

$-1500$

$-1000$

$-151.8-152.2$

f1 (ppm) 

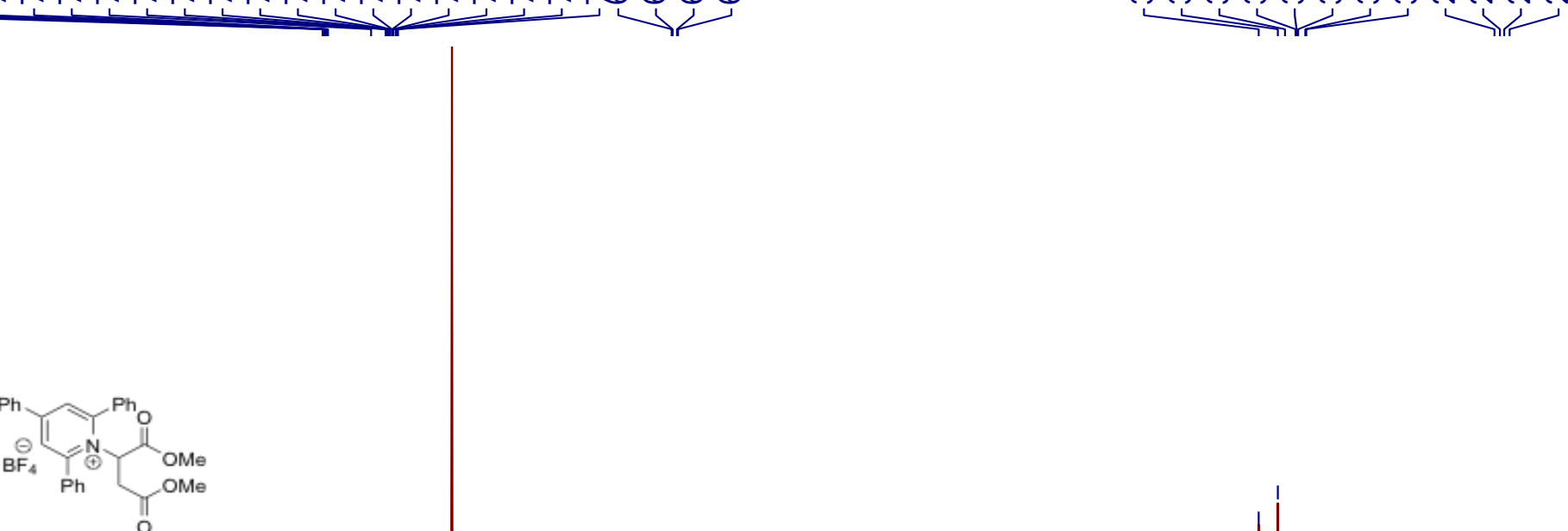

Compound $3 \mathrm{~m}$

${ }^{1} \mathrm{H}$ NMR $\left(400 \mathrm{MHz}, \mathrm{CDCl}_{3}\right)$
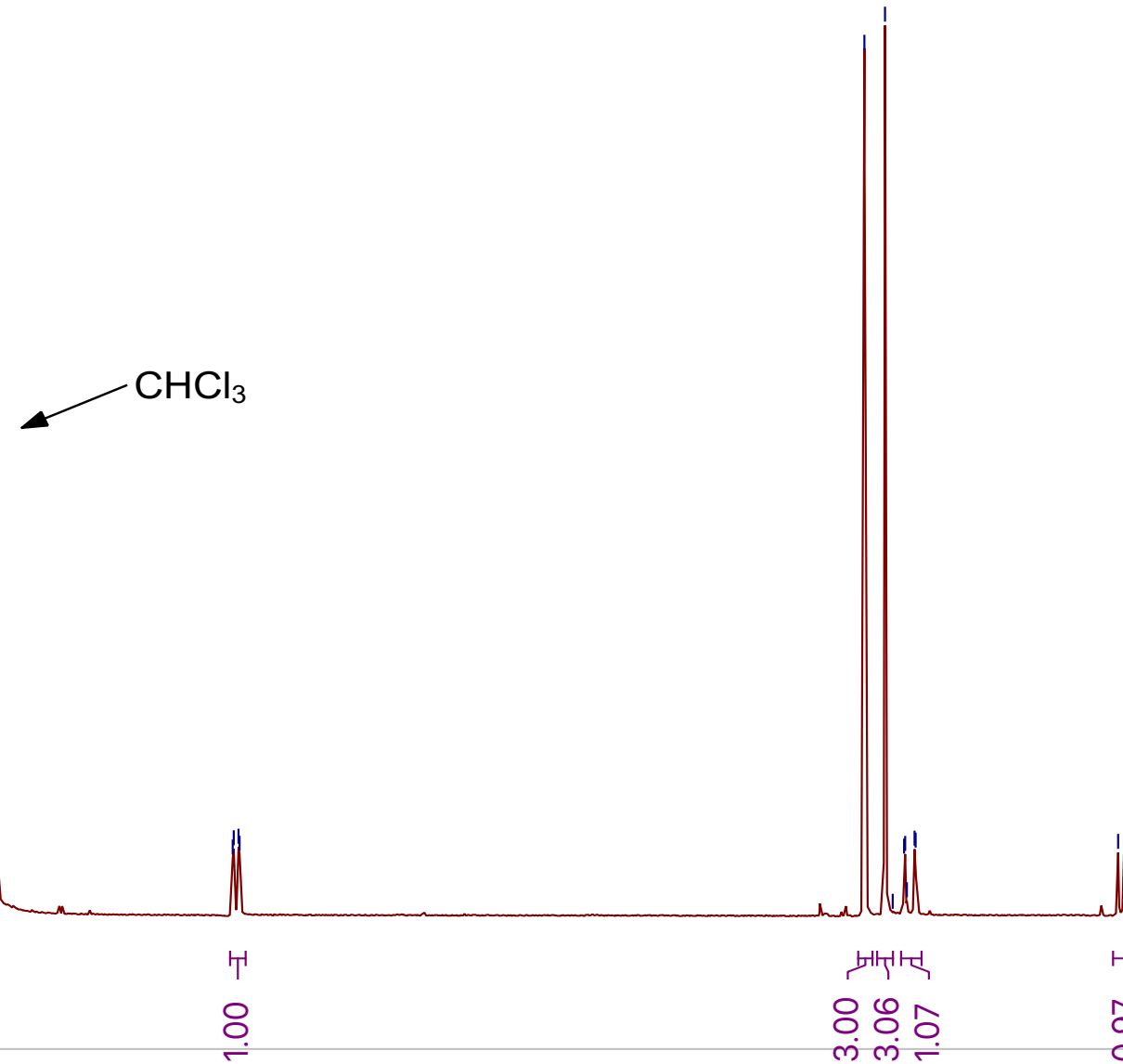

Th,

品

$\stackrel{-}{\circ}$

오오. 


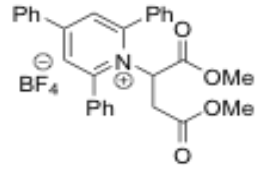

Compound $3 \mathrm{~m}$

${ }^{13} \mathrm{C}$ NMR $\left(101 \mathrm{MHz}, \mathrm{CDCl}_{3}\right)$

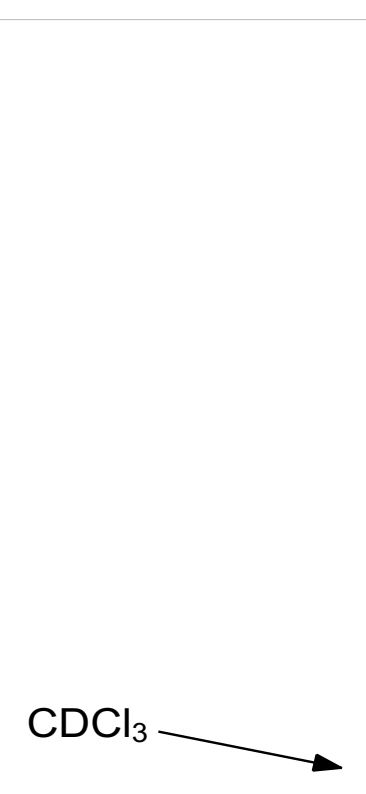




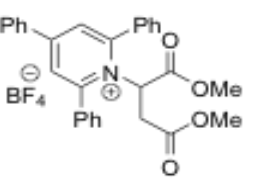

$\infty$

กิ่ กิ่

Compound $3 \mathrm{~m}$

${ }^{19} \mathrm{~F} \mathrm{NMR}\left(376 \mathrm{MHz}, \mathrm{CDCl}_{3}\right.$ )

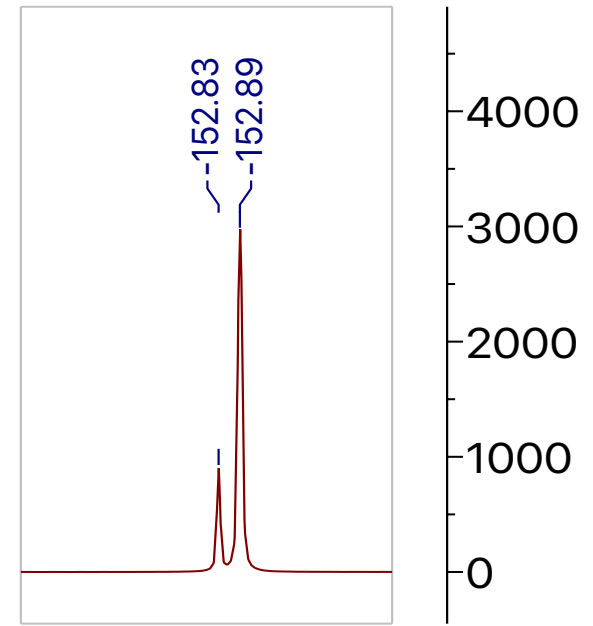

$-3000$

$-2500$

$-2000$

$-1500$

$-152.4 \quad-153.2$

f1 (ppm)

$-1000$

$-500$

0

$\begin{array}{lllllllllllllllllll}-10 & -20 & -30 & -40 & -50 & -60 & -70 & -80 & -90 & -100 & -110 & -120 & -130 & -140 & -150 & -160 & -170 & -180 & -190\end{array}$ 


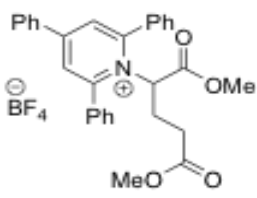

Compound $3 n$

${ }^{1} \mathrm{H}$ NMR $\left(600 \mathrm{MHz}, \mathrm{CDCl}_{3}\right)$

-11000
-10000
-8000
-7000
-6000
-5000
-4000
-3000
-2000
-1000
-0
-1000




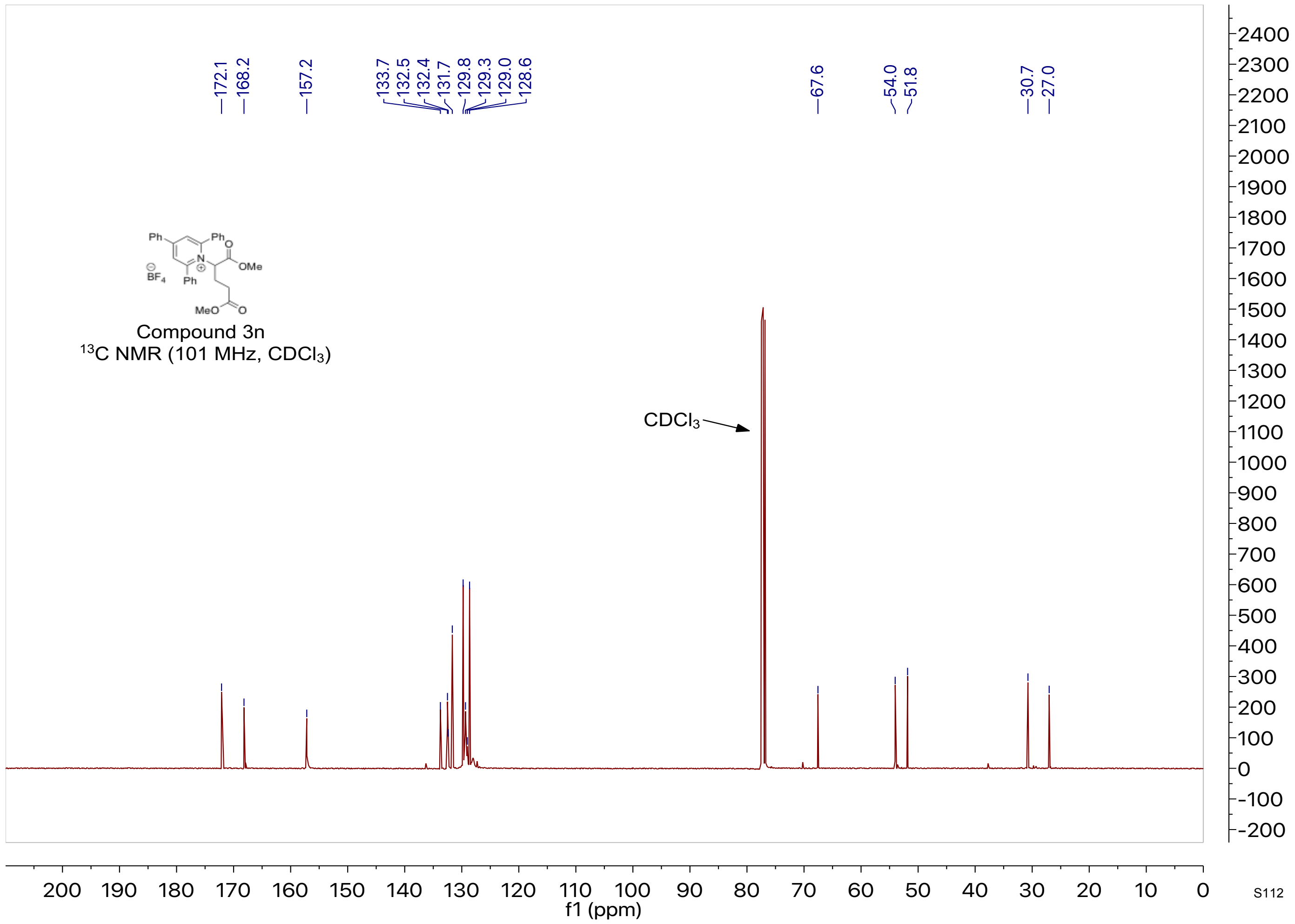




Compound $3 n$

${ }^{19} \mathrm{~F} \mathrm{NMR} \mathrm{(565} \mathrm{MHz,} \mathrm{CDCl}_{3}$ )

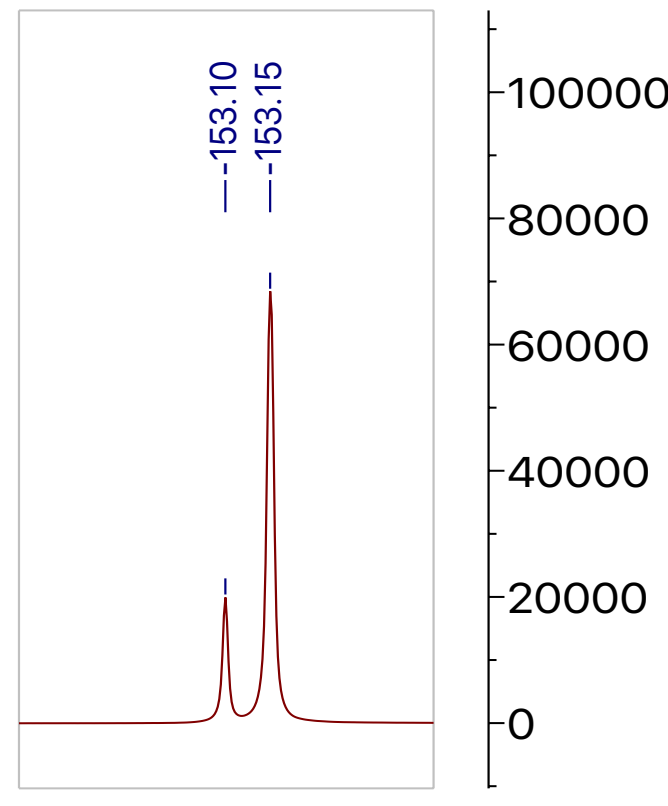

$-153.0$

f1 (ppm)
$-110000$

$-100000$

90000

$-80000$

$-70000$

$-60000$

$-50000$

$-40000$

$-30000$

$-20000$

$-10000$

$-0$

$-10000$

10

$-10$

$-30$

$-50$

$-70$

$-90$

f1 (ppm)
$-110$

$-130$

$-150$

$-170$

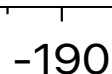

$-190$

$-210$ 

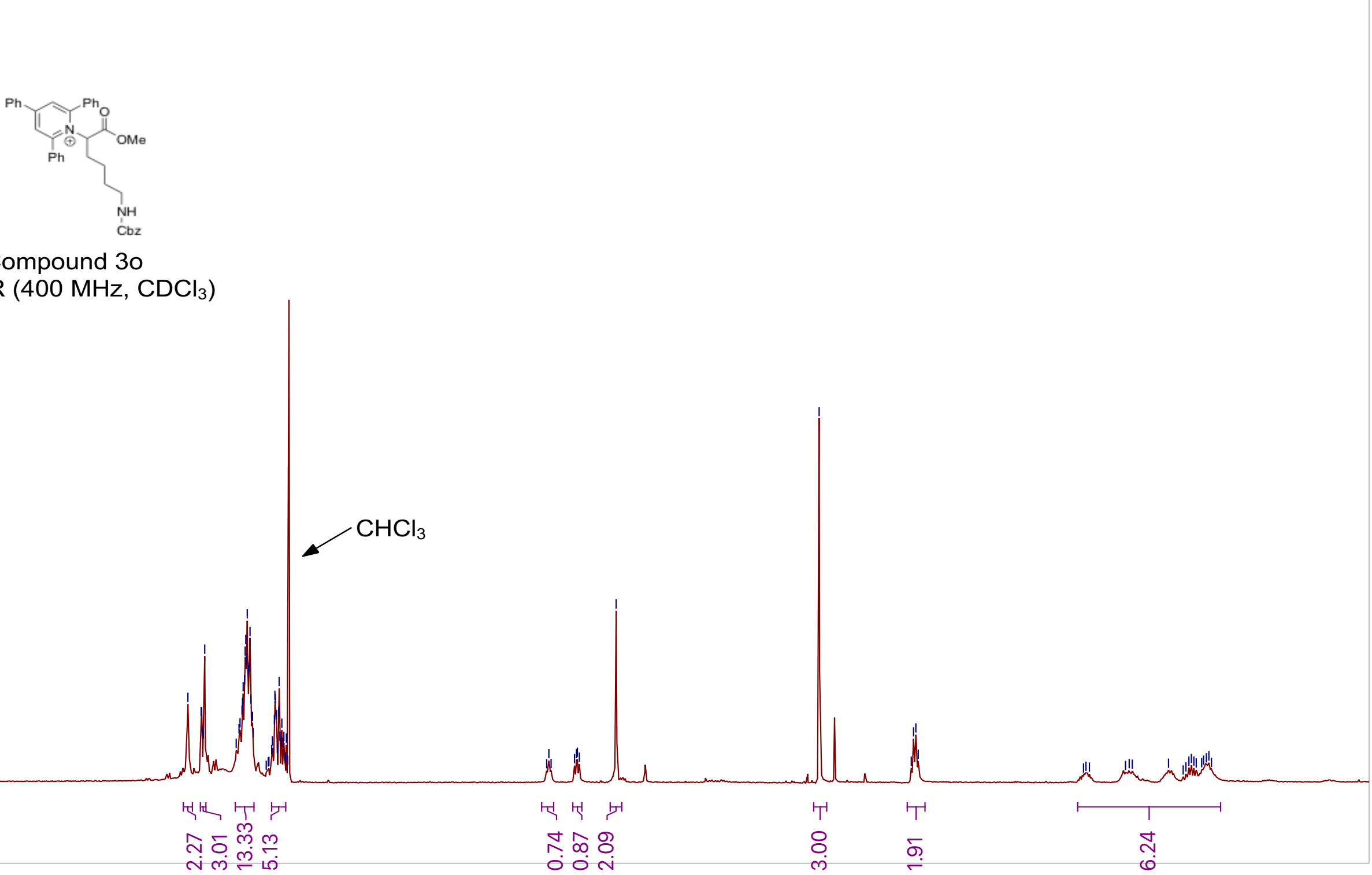

Compound 30

${ }^{1} \mathrm{H}$ NMR $\left(400 \mathrm{MHz}, \mathrm{CDCl}_{3}\right)$
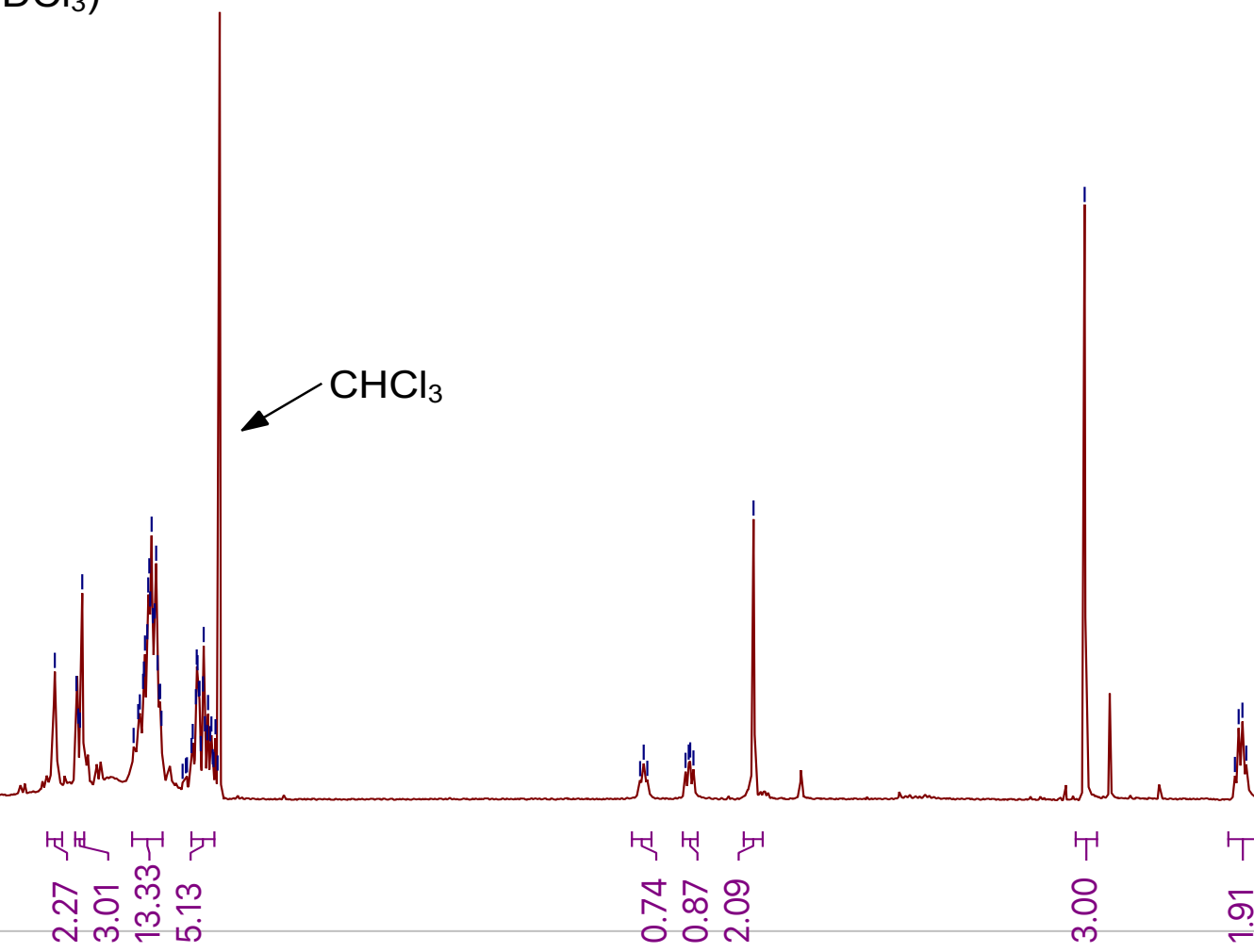


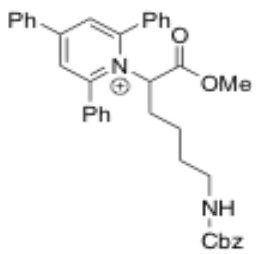

Compound 30

${ }^{13} \mathrm{C} \mathrm{NMR}\left(101 \mathrm{MHz}, \mathrm{CDCl}_{3}\right)$

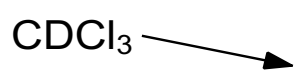


Compound 30

${ }^{19} \mathrm{~F}$ NMR (376 MHz, $\mathrm{CDCl}_{3}$ )

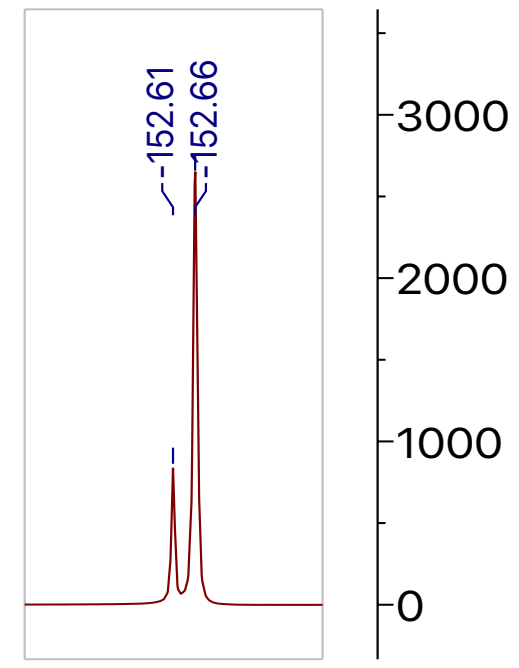

$-152.4-152.7$

f1 (ppm) 


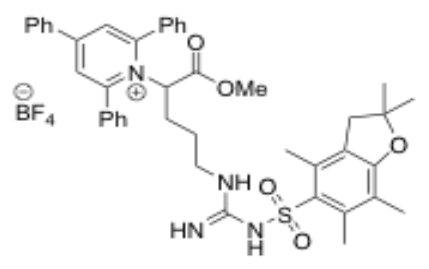

Compound $3 p$

${ }^{1} \mathrm{H}$ NMR $\left(400 \mathrm{MHz}, \mathrm{CDCl}_{3}\right)$

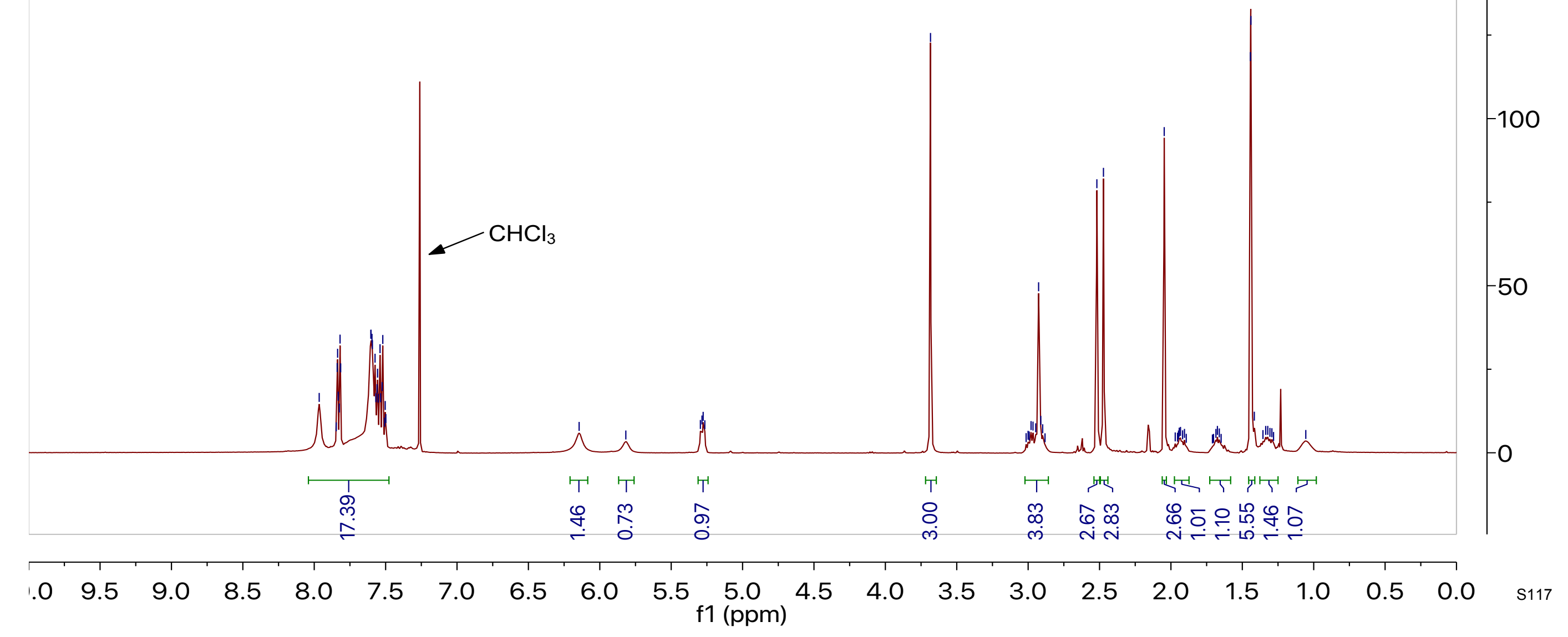




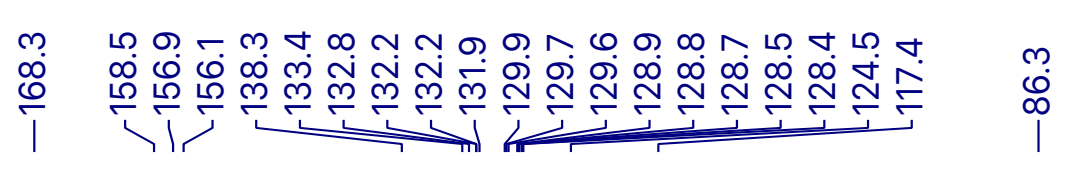

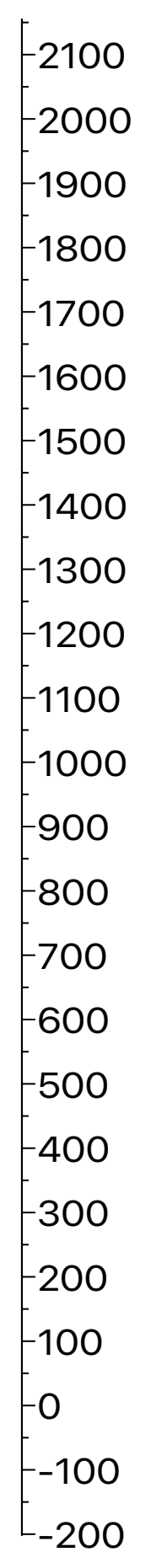

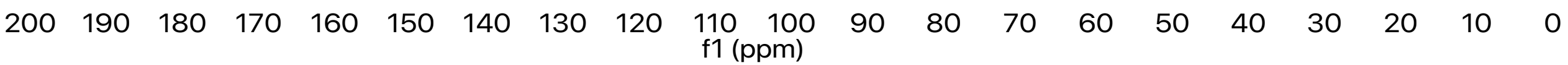


Ph

Compound 3

${ }^{19} \mathrm{~F}$ NMR (376 MHz, $\mathrm{CDCl}_{3}$ )

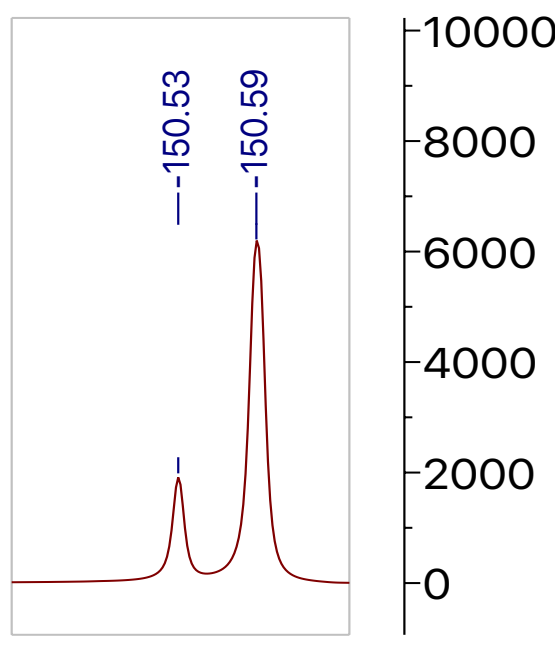

7000

$-6000$

$-5000$

$-4000$

$-3000$

$-150.5-150.6$

f1 (ppm) 


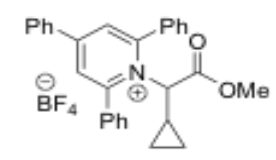

Compound $3 q$

${ }^{1} \mathrm{H}$ NMR (400 $\left.\mathrm{MHz}, \mathrm{CDCl}_{3}\right)$
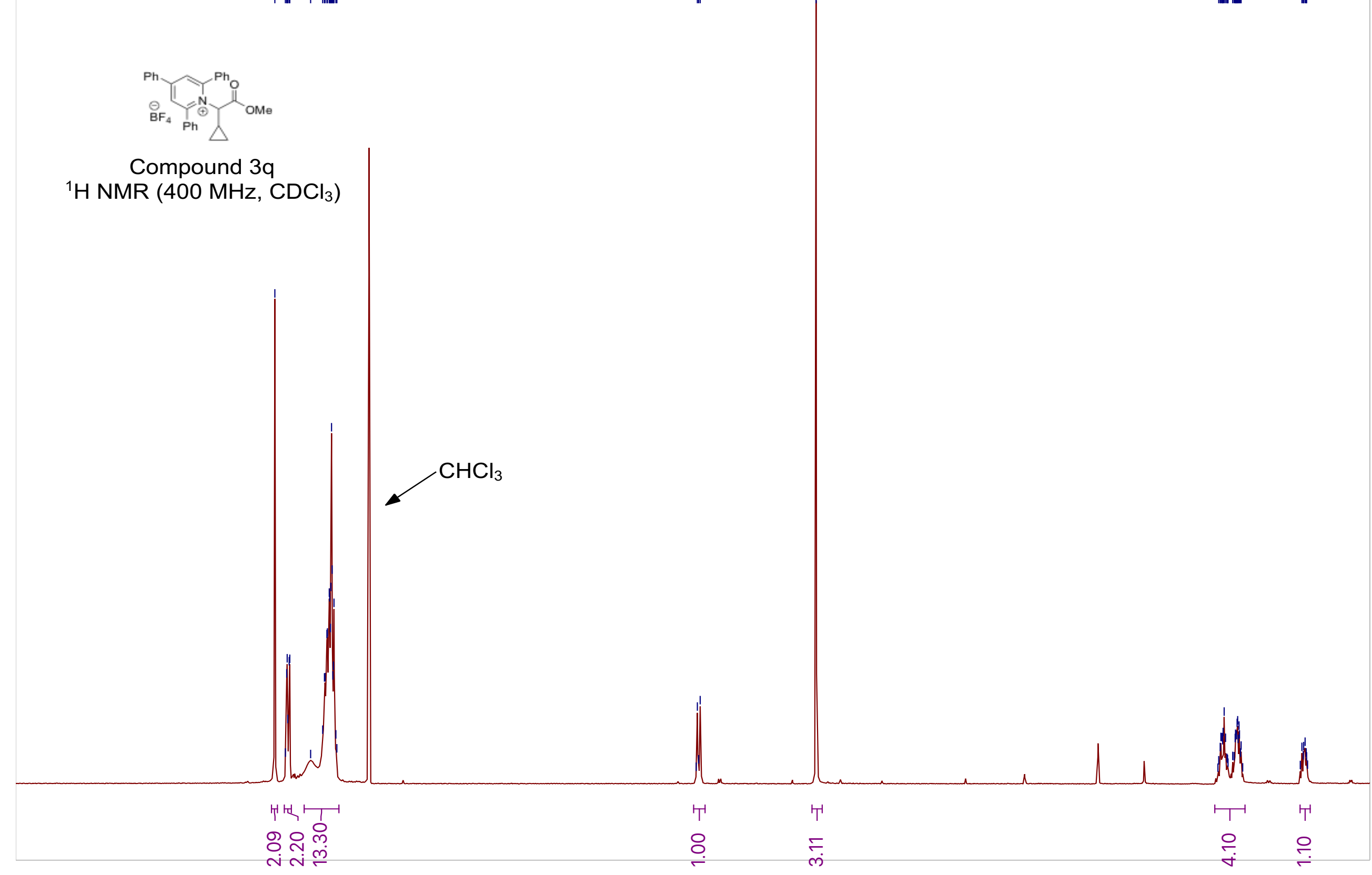


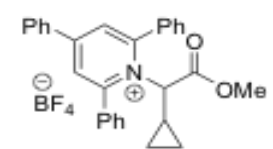

Compound $3 q$

${ }^{13} \mathrm{C}$ NMR $\left(101 \mathrm{MHz}, \mathrm{CDCl}_{3}\right)$

\section{$-1000$}

官 
Ph

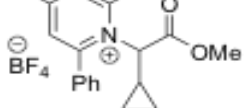

Compound $3 q$

${ }^{19} \mathrm{~F}$ NMR (376 $\mathrm{MHz}, \mathrm{CDCl}_{3}$ )

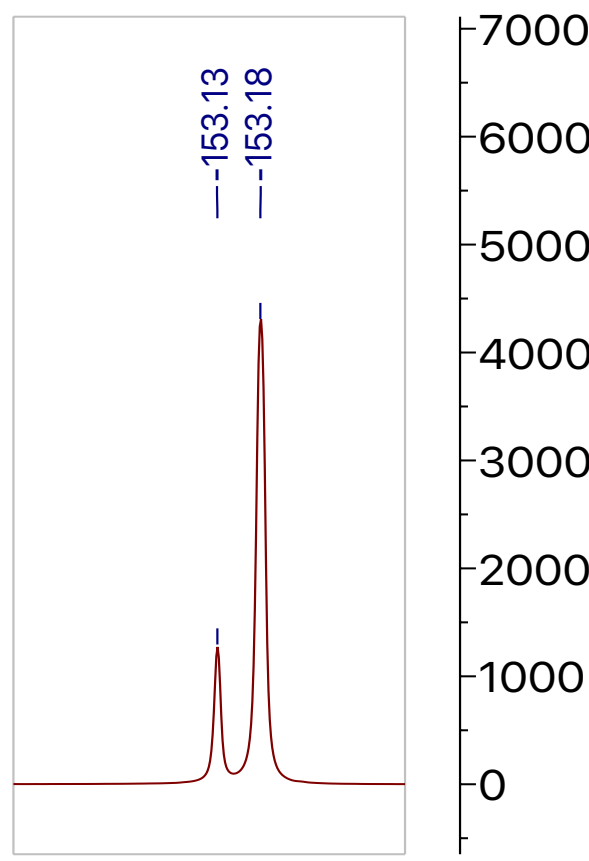

7000
6000
5000
4000
3000
2000
-1000
0

$-5000$

$-4500$

$-4000$

$-3500$

$-3000$

$-2500$

$-2000$

$-1500$

$-1000$

$-153.0 \quad-153.3$

f1 (ppm) 